ESAIM: COCV

Vol. $13, \mathrm{~N}^{\mathrm{O}} 1,2007$, pp. 35-71

DOI: $10.1051 /$ cocv:2007004
ESAIM: Control, Optimisation and Calculus of Variations

www.edpsciences.org/cocv

\title{
LOCAL MINIMIZERS WITH VORTEX FILAMENTS FOR A GROSS-PITAEVSKY FUNCTIONAL
}

\author{
ROBERT L. JERRARD ${ }^{1}$
}

\begin{abstract}
This paper gives a rigorous derivation of a functional proposed by Aftalion and Rivière [Phys. Rev. A 64, 043611 (2001)] to characterize the energy of vortex filaments in a rotationally forced Bose-Einstein condensate. This functional is derived as a $\Gamma$-limit of scaled versions of the Gross-Pitaevsky functional for the wave function of such a condensate. In most situations, the vortex filament energy functional is either unbounded below or has only trivial minimizers, but we establish the existence of large numbers of nontrivial local minimizers and we prove that, given any such local minimizer, the Gross-Pitaevsky functional has a local minimizer that is nearby (in a suitable sense) whenever a scaling parameter is sufficiently small.
\end{abstract}

Mathematics Subject Classification. 35Q40, 35B25, 49Q20.

Received December 13, 2004. Revised August 22, 2005.

\section{INTRODUCTION}

This paper presents a rigorous derivation of a reduced energy proposed in the physics literature to explain the geometry of vortex filaments in rotationally forced Bose-Einstein condensates as observed in recent experiments, see for example [17], [19]. A condensate is described by a wave function, and the wave function in the zerotemperature limit is expected to be a critical point of the Gross-Pitaevsky energy

$$
F(\psi)=\int \frac{|\nabla \psi|^{2}}{2}-a\langle V, j \psi\rangle+b W(x)|\psi|^{2}+c|\psi|^{4}
$$

in a set of the form $\left\{\psi \in H^{1}\left(\mathbb{R}^{3} ; \mathbb{C}\right):\|\psi\|_{L^{2}}^{2}=m\right\}$. Here $a, b$ are positive constants, $W: \mathbb{R}^{3} \rightarrow[0, \infty)$ represents a confining potential, $V$ represents the forcing, and $j \psi$ is the momentum density of the condensate, defined in (3.2). The term $-a\langle V, j \psi\rangle$ is small (i.e., negative with large absolute value) if roughly speaking the momentum density of the condensate is parallel to with the velocity field imposed on the condensate by the rotational forcing; the notation denotes the dual pairing between a vector and a 1-form.

Various physical attributes of a Bose-Einstein condensate are encoded in the wave function $\psi$ : for example, $|\psi|^{2}$ represents the density. A vortex filament may be thought of as a 1-dimensional curve in $\mathcal{D}$ along which the complex-valued wave function $\psi$ has a phase singularity.

In this paper I will always consider a model case, in which $W$ is a paraboloid and $V$ is generated by rotation around one of the axes, a situation studied in a recent paper of Aftalion and Rivière [3]. These authors argue

Keywords and phrases. Gross-Pitaevsky, vortices, Gamma-convergence, Thomas-Fermi limit, rectifiable currents.

1 Math Department, University of Toronto, Toronto, ON M5S 3G3, Canada; rjerrard@math.toronto.edu

(C) EDP Sciences, SMAI 2007 
that minimizers of $F$ with the constraint $\|\psi\|_{L^{2}}^{2}=m$ will be exponentially small away from a set of the form

$$
\mathcal{D}:=\left\{x \in \mathbb{R}^{3}: \rho(x)>0\right\}
$$

where

$$
\rho(x)=1-\left(a_{1}^{2} x_{1}^{2}+a_{2}^{2} x_{2}^{2}+a_{3}^{2} x_{3}^{2}\right), \text { for certain constants } a_{i}>0 .
$$

Moreover, in $\mathcal{D}$ the wave function is expected to be close to a critical point in the space $H_{0}^{1}(\mathcal{D} ; \mathbb{C}):=\{u \in$ $H^{1}(\mathcal{D} ; \mathbb{C}): u=0$ on $\left.\partial \mathcal{D}\right\}$ of a functional that can be written

$$
G_{\Omega_{\varepsilon}}^{\varepsilon}(u):=\int_{\mathcal{D}} \frac{1}{2}|\nabla u|^{2}-\Omega_{\varepsilon}\langle V, j u\rangle+\frac{1}{4 \varepsilon^{2}}\left(\rho-|u|^{2}\right)^{2} \mathrm{~d} x
$$

after a suitable nondimensionalization. Here $\Omega_{\varepsilon}$ is a real parameter corresponding to the rate of rotation and

$$
V(x):=-x_{2} e_{1}+x_{1} e_{2}
$$

where $e_{i}$ denotes the standard unit vector in the $i$ th direction. The value of $\varepsilon$ in the experiments cited above is on the order of $10^{-2}$ or smaller.

Aftalion and Rivière in [3] give a formal derivation, taking $G_{\Omega_{\varepsilon}}^{\varepsilon}$ as their starting point, of a functional $E_{\Omega}$ that describes the energy of a vortex filament when $\varepsilon \ll 1$ and $\Omega \approx \Omega_{\varepsilon} /|\ln \varepsilon|$. Such a filament can be represented by an oriented curve, i.e. a Lipschitz function $X:(a, b) \subset \mathbb{R} \rightarrow \mathcal{D}$. With this notation,

$$
E_{\Omega}(X)=\int_{a}^{b} \rho(X(s))|\dot{X}(s)|-\Omega \rho^{2}(X(s)) e_{3} \cdot \dot{X}(s) \mathrm{d} s .
$$

For topological reasons vortex lines have no boundary in $\mathcal{D}$, which means that

$$
\text { either } X(a)=X(b) \quad \text { or } X(a), X(b) \in \partial \mathcal{D}
$$

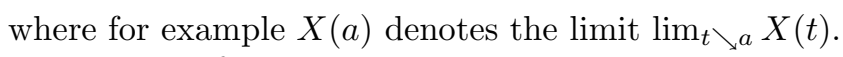

The goal of this paper is to make precise the relationship between $G_{\Omega_{\varepsilon}}^{\varepsilon}$ and $E_{\Omega}$, and to demonstrate some ways in which the simpler functional $E_{\Omega}$ captures the behavior of vortex lines in certain critical points of $G_{\Omega_{\varepsilon}}^{\varepsilon}$ when $\varepsilon \ll 1$ and $\Omega$ and $\Omega_{\varepsilon}$ are related in a suitable way. I define a critical value

$$
\Omega_{1}:=\inf \left\{\Omega>0: \exists X \text { satisfying (1.7), and such that } E_{\Omega}(X)<0\right\}
$$

such that for $\Omega<\Omega_{1}$, the global minimizer of $E_{\Omega}$ is vortex-free. It will follow from Lemma 7 that $\Omega_{1}>0$. One of the main results shows that for corresponding values $\Omega_{\varepsilon}$ - more precisely for $\Omega_{\varepsilon}=\Omega|\ln \varepsilon|\left(1+\left(\frac{a_{1}}{a_{2}}\right)^{2}\right)$ - the global minizers of $G_{\Omega_{\varepsilon}}^{\varepsilon}$ are asymptotically vortex free as $\varepsilon \rightarrow 0$. The other main results identify another critical value $\Omega_{0}<\Omega_{1}$, defined in (4.16), such that for $\Omega>\Omega_{0}$, both $E_{\Omega}$ and $G_{\Omega_{\varepsilon}}^{\varepsilon}, \varepsilon \ll 1$ have nontrivial local minimizers, with the corresponding vortex filaments in some sense close to each other. Here and throughout this paper, $\Omega$ and $\Omega_{\varepsilon}$ are related as above. Note that for $\Omega \in\left(\Omega_{0}, \Omega_{1}\right)$ there exist stable vortex filaments with positive energy, for both $E_{\Omega}$ and $G_{\Omega_{\varepsilon}}^{\varepsilon}, \varepsilon \ll 1$.

In all these results, "vorticity" is identified as follows: as in [3], I will write a wave function $u_{\varepsilon}$ as a product $\eta_{\varepsilon} v_{\varepsilon}$, where $\eta_{\varepsilon}$ is a nearly optimal vortex-free profile (constructed at the beginning of Sect. 6) and $\left|v_{\varepsilon}\right| \approx 1$. To say that $\eta_{\varepsilon}$ is vortex-free means that it has the form $\eta_{\varepsilon}=f_{\varepsilon} e^{i S_{\varepsilon}}$, where $f_{\varepsilon}>0$ in $\mathcal{D}$ and $S_{\varepsilon}$ is real-valued. Consequently, $u_{\varepsilon}$ and $v_{\varepsilon}$ have exactly the same phase singularities, and because $\left|v_{\varepsilon}\right| \approx 1$, the asymptotic phase singularities of $v_{\varepsilon}$ can be identified by finding limits of the Jacobians $J v_{\varepsilon}$, see for example [12]. Thus theorems about the vorticity of $u_{\varepsilon}$ will be stated as results about Jacobians of the auxiliary functions $v_{\varepsilon}=u_{\varepsilon} / \eta_{\varepsilon}$.

The language of geometric measure theory is needed to make precise for example the sense in which $E_{\Omega}$ has local minimizers, or the sense in which the vorticity $J v_{\varepsilon}$ associated with a wave function $u_{\varepsilon}$ is close to a limiting 
vortex filament. The relevant material is summarized in Section 3, where I review some general background and then reformulate $E_{\Omega}$ as a functional on certain weighted spaces of rectifiable currents. I also introduce some weighted norms and seminorms and develop some facts about these spaces of currents, including compactness and density properties.

Section 4 contains the proofs of Theorem 1 and Corollary 1, guaranteeing the existence for $\Omega>\Omega_{0}$ of large numbers of local minimizers of $E_{\Omega}$, with respect to the seminorms introduced in Section 3.

Section 5 recalls some estimates relating the Jacobian and the Ginzburg-Landau energy and proves a new estimate in a similar vein, see Lemma 9. Although this estimate is an easy reformulation of known results, it is useful and may be of independent interest. Section 6 uses this Jacobian estimate to prove (see Th. 2) that $E_{\Omega}$ arises as the $\Gamma$-limit of the functionals

$$
u \mapsto \frac{1}{|\ln \varepsilon|}\left(G_{\Omega_{\varepsilon}}^{\varepsilon}(u)-G_{\Omega_{\varepsilon}}^{\varepsilon}\left(\eta_{\varepsilon}\right)\right)
$$

under certain scaling assumptions, where as above $\eta_{\varepsilon}$ is a vortex-free profile and $\Omega_{\varepsilon}=\Omega|\ln \varepsilon|\left(1+\left(\frac{a_{1}}{a_{2}}\right)^{2}\right)$. The same theorem also contains some compactness assertions connected to the $\Gamma$-limit. These are quite delicate, owing to the degeneracy of $\rho$ near $\partial \mathcal{D}$ and the formation of boundary layers.

Section 7 contains the proof of Theorem 3, in which it is shown that if $\Omega$ is such that the minimizer of $E_{\Omega}$ is trivial, then minimizers of $G_{\Omega_{\varepsilon}}^{\varepsilon}$ are asymptotically vortex-free as $\varepsilon \rightarrow 0$. Section 8 presents the proof of 4 , which shows roughly speaking that if $X$ is a local minimizer of $E_{\Omega}$ in the sense of Section 4, then for sufficiently small $\varepsilon$ there exist local minimizers $u_{\varepsilon}$ of $G_{\Omega}^{\varepsilon}$ with vorticity close to $X$ in the sense that $J v_{\varepsilon}-X$ is small with respect to the appropriate seminorms. Finally, Section 9 contains the proofs of some technical facts, mainly auxiliary results about the spaces of integral currents used in this paper.

It is not hard to see that the limiting energy $E_{\Omega}$ is either nonnegative or unbounded below, depending on the value of $\Omega$. This reflects the fact that in the derivation of $E_{\Omega}$, lower-order terms that vanish in the limit $\varepsilon \rightarrow 0$ include quadratic interaction terms that, for small but finite $\varepsilon$, make it energetically unfavorable to increase the number of vortex lines arbitrarily. These terms can safely be neglected when studying suitable local minimizers of $G_{\Omega_{\varepsilon}}^{\varepsilon}$, as is done in Theorem 4, since these local minimizers can be sought in subsets of $H_{0}^{1}(\mathcal{D} ; \mathbb{C})$ in which the vorticity is controlled. A more careful accounting of these interaction terms would be required for a description of global minimizers of $G_{\Omega_{\varepsilon}}^{\varepsilon}$ in the parameter range $\Omega>\Omega_{1}$ in which $E_{\Omega}$ is unbounded below.

Related works include the pioneering book of Bethuel, Brezis and Hélein [7] on Ginzburg-Landau vortices in two dimensions, and subsequent work by Andre and Shafrir [6] and Lassoued and Mironescu [15] among others, on the corresponding weighted problem; the analysis of asymptotics of Ginzburg-Landau minimizers in 3 and higher dimensions, initiated by Rivière [18] and subsequently explored in great depth by a large number of researchers; work of Kohn and Sternberg [14] that developed the use of $\Gamma$-convergence results to prove the existence of local minimizers of functionals containing a small parameter, and recent papers [13, 16] that carry out this sort of argument for the Ginzburg-Landau functional on certain bounded domains in 3 dimensions, with and without magnetic field.

\section{NOTATION AND PRELIMINARIES}

\subsection{General notation}

Recall that the domain $\mathcal{D}$ and the function $\rho$ are defined in (1.2) and (1.3) respectively. I always assume that $a_{1} \leq a_{2}$ and $a_{3} \leq a_{2}$, where $a_{1}, a_{2}, a_{3}$ are the parameters in the definitions of $\rho, \mathcal{D}$. The first assumption does not entail any loss of generality, but the second does, since the $x_{3}$ axis is distinguished as the axis of rotation (recall (1.5)). The assumption $a_{3} \leq a_{2}$, which is needed only for Lemma 7, could almost surely be removed, but in any case it is consistent with most physical experiments, in which the condensates are cigar-shaped, rather than pancake-shaped. 
It is useful to define

$$
\begin{aligned}
\mathcal{D}_{0} & :=\left\{x \in \mathcal{D}: x_{1}=0\right\}, \\
\mathcal{D}_{0}{ }^{+} & :=\left\{x \in \mathcal{D}_{0}: x_{2} \geq 0\right\} .
\end{aligned}
$$

Since $a_{1} \leq a_{2}, \mathcal{D}_{0}$ is the thinnest cross-section of $\mathcal{D}$ that contains the distinguished $x_{3}$ axis. I will often need the function $p: \mathcal{D} \rightarrow \mathcal{D}_{0}{ }^{+}$defined by

$$
p(x)=p\left(x_{1}, x_{2}, x_{3}\right):=\left(0,\left(\frac{a_{1}^{2}}{a_{2}^{2}} x_{1}^{2}+x_{2}^{2}\right)^{1 / 2}, x_{3}\right)=\left(p^{1}(x), p^{2}(x), p^{3}(x)\right) .
$$

Note that $\rho(p(x))=\rho(x)$ for all $x \in \mathcal{D}$.

Throughout this paper I use the convention that repeated indices are summed over.

$W \subset \subset U$ means that $\bar{W}$ is a compact subset of $U$.

The characteristic function of a set $W$ is denoted by $\chi_{W}$, so that $\chi_{W}(x)=1$ if and only if $x \in W$.

For $v, w \in \mathbb{C}$, I write $(v, w):=\frac{1}{2}(v \bar{w}+\bar{v} w)$ for the real inner product. Note that

$$
(i v, w)=\operatorname{det}\left(\begin{array}{cc}
\mathfrak{R e} v & \mathfrak{R e} w \\
\mathfrak{I m} v & \mathfrak{I m} w
\end{array}\right) .
$$

The Ginzburg-Landau energy density will be denoted by

$$
e_{\varepsilon}(u):=\frac{1}{2}|\nabla u|^{2}+\frac{1}{4 \varepsilon^{2}}\left(|u|^{2}-1\right)^{2} .
$$

Notation relating to currents and differential forms is introduced in Section 3.

\subsection{A lemma}

The following easy lemma shows that individual terms in $G_{\Omega_{\varepsilon}}^{\varepsilon}$ are bounded above and below. It will be used a number of times.

Lemma 1. There exists $C_{\varepsilon}$ depending only on $\varepsilon \Omega_{\varepsilon}$ and the parameters in the definitions of $\rho, \mathcal{D}$, such that

$$
\int \frac{1}{2}\left|\nabla u_{\varepsilon}\right|^{2}+\frac{1}{4 \varepsilon^{2}}\left(\rho-\left|u_{\varepsilon}\right|^{2}\right)^{2} \mathrm{~d} x+\Omega_{\varepsilon} \int\left|j u_{\varepsilon}\right| \mathrm{d} x \leq C_{\varepsilon}\left(\Omega_{\varepsilon}{ }^{2}+G_{\Omega_{\varepsilon}}^{\varepsilon}\left(u_{\varepsilon}\right)\right)
$$

for every $u_{\varepsilon} \in H^{1}(\mathcal{D} ; \mathbb{C})$ and every $\varepsilon \in(0,1]$.

Throughout this paper the product $\varepsilon \Omega_{\varepsilon}$ will always be uniformly bounded, so that in effect $C_{\varepsilon}$ will be independent of $\varepsilon$.

Proof. First note that

$$
\begin{aligned}
\left|\Omega_{\varepsilon} V \cdot j u_{\varepsilon}\right| & \leq C \Omega_{\varepsilon}\left|j u_{\varepsilon}\right| \\
& \leq \frac{1}{4}\left|\nabla u_{\varepsilon}\right|^{2}+C \Omega_{\varepsilon}{ }^{2}\left(\left|u_{\varepsilon}\right|^{2}-\rho\right)+C \Omega_{\varepsilon}{ }^{2} \rho \\
& \leq \frac{1}{4}\left|\nabla u_{\varepsilon}\right|^{2}+\frac{1}{8 \varepsilon^{2}}\left(\left|u_{\varepsilon}\right|^{2}-\rho\right)^{2}+C \Omega_{\varepsilon}{ }^{2}\left(\rho+\Omega_{\varepsilon}{ }^{2} \varepsilon^{2}\right) \\
& \leq \frac{1}{4}\left|\nabla u_{\varepsilon}\right|^{2}+\frac{1}{8 \varepsilon^{2}}\left(\left|u_{\varepsilon}\right|^{2}-\rho\right)^{2}+C_{\varepsilon} \Omega_{\varepsilon}{ }^{2} .
\end{aligned}
$$

Thus the bound on $\Omega_{\varepsilon} \int\left|j u_{\varepsilon}\right|$ follows from the bound on the other term. The above inequality also implies that

$$
\frac{1}{2}\left|\nabla u_{\varepsilon}\right|^{2}-\Omega_{\varepsilon} V \cdot j u_{\varepsilon}+\frac{1}{4 \varepsilon^{2}}\left(\rho-\left|u_{\varepsilon}\right|^{2}\right)^{2}+C_{\varepsilon} \Omega_{\varepsilon}{ }^{2} \geq \frac{1}{2}\left(\frac{1}{2}\left|\nabla u_{\varepsilon}\right|^{2}+\frac{1}{4 \varepsilon^{2}}\left(\rho-\left|u_{\varepsilon}\right|^{2}\right)^{2}\right) .
$$

The conclusion now follows by integrating this inequality over $\mathcal{D}$. 


\section{FORMS AND CURRENTS}

It is convenient to reformulate $E_{\Omega}$ as a functional acting on a weighted space of rectifiable 1-currents in $\mathcal{D}$. All the currents that occur in this paper admit simple representations, in terms of vector-valued measures or skew-gradients of functions of bounded variation, and readers unfamiliar with geometric measure theory are encouraged to consult Subsection 3.4, where these simple representations are discussed.

\subsection{Basic definitions}

I write $\Lambda^{k} \mathbb{R}^{n}$ to denote the space of $k$-covectors on $\mathbb{R}^{n}$, a vector space with basis $\left\{\mathrm{d} x^{\alpha_{1}} \wedge \ldots \wedge \mathrm{d} x^{\alpha_{k}}: 1 \leq\right.$ $\left.\alpha_{1}<\ldots<\alpha_{k} \leq n\right\}$. I define an inner product on $\Lambda^{k} \mathbb{R}^{n}$ by requiring that this basis be orthonormal, and I write $\phi \cdot \omega$ for the inner product of $k$-covectors $\phi$ and $\omega$. I also use the notation $|\phi|=(\phi, \phi)^{1 / 2}$.

Similarly, $\Lambda_{k} \mathbb{R}^{n}$ denotes the space of $k$-vectors, with the basis $\left\{e_{\alpha_{1}} \wedge \ldots \wedge e_{\alpha_{k}}: 1 \leq \alpha_{1}<\ldots<\alpha_{k} \leq n\right\}$. The dual pairing between vectors and covectors is denoted by $\langle\cdot, \cdot\rangle$, and the bases for $\Lambda^{k} \mathbb{R}^{n}$ and $\Lambda_{k} \mathbb{R}^{n}$ are assumed to be dual, so that for example $\left\langle\phi^{i} \mathrm{~d} x^{i}, T_{j} e_{j}\right\rangle=\sum \phi^{i} T_{i}$ when $k=1$. The Hodge operator $\star: \Lambda^{k} \mathbb{R}^{n} \rightarrow \Lambda_{n-k} \mathbb{R}^{n}$ is defined by stipulating that

$$
\langle\star \phi, \omega\rangle=\phi \wedge \omega \quad \text { for all } \phi \in \Lambda^{k} \mathbb{R}^{n}, \omega \in \Lambda^{n-k} \mathbb{R}^{n} .
$$

A differential $k$-form on an open set $U \subset \mathbb{R}^{n}$ is a map $\phi: U \rightarrow \Lambda^{k} \mathbb{R}^{n}$. I define exterior differentiation and pullback as usual, so that for example if $\phi=\phi^{i} \mathrm{~d} x^{i}$ is a one-form, then $\mathrm{d} \phi=\phi_{x_{j}}^{i} \mathrm{~d} x^{j} \wedge \mathrm{d} x^{i}$. And for $\eta=\left(\eta^{1}, \ldots, \eta^{n}\right): W \subset \mathbb{R}^{m} \rightarrow U$ and $\phi$ a 1 -form on $U$ as above, the pullback $\eta^{\#} \phi$ is a 1 -form on $W$ defined by $\eta^{\#} \phi=\phi^{i}(\eta(y)) \frac{\partial \eta^{i}}{\partial y_{j}} \mathrm{~d} y^{j}$.

A $k$-dimensional current $T$ on an open set $U \subset \mathbb{R}^{n}$ is a bounded linear functional on the space of smooth $k$-forms with compact support in $U$. The boundary of a $k$-dimensional current $T$ is the $k-1$ dimensional current $\partial T$ defined by $\partial T(\phi)=T(d \phi)$. The image of a current $T$ under a mapping $\eta$ is defined by $\eta_{\#} T(\phi)=T\left(\eta^{\#} \phi\right)$.

A current $T$ is said to have locally finite mass in $U \subset \mathbb{R}^{n}$ if it can be represented in the form

$$
T(\phi)=\int_{U}\langle\phi, \vec{T}\rangle \mathrm{d}\|T\| \quad \text { for } \phi \in C_{c}^{\infty}\left(U ; \Lambda^{k} \mathbb{R}^{n}\right)
$$

where $\|T\|$ is a nonnegative Radon measure, locally finite in $U$, and $\vec{T}$ is a $\|T\|$ measurable function taking values in $\Lambda_{k} \mathbb{R}^{n}$, normalized by requiring that $|\vec{T}|=1$ almost everywhere. I use the notation

$$
\mathcal{M}_{k}(U):=\{k \text {-dimensional currents with locally finite mass in } U\} \text {. }
$$

A current $T \in \mathcal{M}_{k}(U)$ is integer multiplicity rectifiable if there exists a $k$ rectifiable ${ }^{1}$ set $\Gamma$ and a $\mathcal{H}^{k}$-measurable function $m: \Gamma \rightarrow \mathbb{Z}^{+}$such that for $\mathcal{H}^{k}$ almost every $x,|\vec{T}(x)|=1$ and $\vec{T}(x)$ orients the approximate tangent space $\operatorname{ap} T_{x} \Gamma$, and if

I will write

$$
\int \psi \mathrm{d}\|T\|=\int_{\Gamma} \psi(x) m(x) \mathcal{H}^{k}(\mathrm{~d} x) \quad \text { for all } \psi \in C_{c}(U ; \mathbb{R})
$$

$$
\mathcal{R}_{k}(U):=\left\{T \in \mathcal{M}_{k}(U): T \text { is rectifiable }\right\} \text {. }
$$

For currents defined on the domain $\mathcal{D}$, we will often need the spaces

$$
\mathcal{M}_{k, \rho}(\mathcal{D}):=\left\{T \in \mathcal{M}_{k}(\mathcal{D}): \int \rho \mathrm{d}\|T\|<\infty\right\}, \quad \mathcal{R}_{k, \rho}(\mathcal{D}):=\mathcal{R}_{k}(\mathcal{D}) \cap \mathcal{M}_{k, \rho}(\mathcal{D})
$$

\footnotetext{
${ }^{1}$ Recall that a set $\Gamma \subset \mathbb{R}^{n}$ is $k$ rectifiable if there exists a set $M_{0}$ such that $\mathcal{H}^{k}\left(M_{0}\right)=0$ and $C^{1} k$-dimensional submanifolds $M_{i} \subset \mathbb{R}^{n}, i=1,2, \ldots$ such that $\Gamma \subset \cup_{i=0}^{\infty} M_{i}$. In particular if $\Gamma$ is $k$ rectifiable, then $\Gamma$ has an approximate tangent space ap $T_{x} \Gamma$ at $\mathcal{H}^{k}$ a.e. $x \in \Gamma$, see for example [11] Vol. 1, Section 2.1.4.
} 
In particular we will often work with $\mathcal{R}_{1, \rho}(\mathcal{D})$. Currents in this space have a fairly simple description as a sum of oriented Lipschitz curves, see Lemma 3. Finally, a circle ${ }^{\circ}$ will be used to denote spaces of currents with no boundary, for example

$$
\stackrel{\circ}{\mathcal{M}}_{1, \rho}(\mathcal{D})=\left\{T \in \mathcal{M}_{1, \rho}(\mathcal{D}): \partial T=0 \text { in } \mathcal{D}\right\}, \quad \stackrel{\circ}{\mathcal{R}}_{1, \rho}(\mathcal{D})=\mathcal{R}_{1, \rho}(\mathcal{D}) \cap \dot{\mathcal{M}}_{1, \rho}(\mathcal{D}) .
$$

\subsection{Jacobians}

Given an open subset $U \subset \mathbb{R}^{m}, m \geq 2$ and a function $u \in H^{1}(U ; \mathbb{C})$, we define the 1-form

$$
j u=\left(i u, u_{x_{j}}\right) \mathrm{d} x^{j} .
$$

If $u$ is a wave function then $j u$ corresponds to its momentum density. Note that if $u$ is written locally in the form $u=\rho e^{i \phi}$, then $j u=\rho^{2} \mathrm{~d} \phi$. we also define the Jacobian

$$
J u=\sum_{j<k}\left(i u_{x_{j}}, u_{x_{k}}\right) \mathrm{d} x^{j} \wedge \mathrm{d} x^{k} .
$$

From (2.4) one easily sees that $J u$ is just the pullback by $u$ of the standard area form $\mathrm{d} x$ on $\mathbb{C}$, that is $J u=u^{\#}(\mathrm{~d} x)$.

It is convenient to associate with $j u$ and $J u$ a $m-1$-current and $m-2$-current respectively, defined by

$$
\star j u(\omega)=\int_{\mathcal{D}} \omega \wedge j u, \quad \star J u(\phi)=\int_{\mathcal{D}} \phi \wedge J u .
$$

One easily verifies that $J u=\frac{1}{2} \mathrm{~d} j u$, and this implies that $\star J u=\frac{1}{2} \partial(\star j u)$.

\subsection{Some norms and seminorms}

The mass of a current $T \in \mathcal{M}_{k}(U)$ is given by

$$
\mathbf{M}_{U}(T):=\|T\|(U)=\sup \left\{T(\phi): \phi \in C_{c}^{\infty}\left(U ; \Lambda^{k} \mathbb{R}^{n}\right),\|\phi\|_{\infty} \leq 1\right\} .
$$

According to my conventions, $\mathbf{M}_{U}(T)$ can be infinite for $T \in \mathcal{M}_{k}(U)$. For currents $T \in \mathcal{M}_{k}(\mathcal{D})$ I will often need the weighted mass $\mathbf{M}_{\rho}(T)$, defined by

$$
\mathbf{M}_{\rho}(T):=\int_{\mathcal{D}} \rho \mathrm{d}\|T\|=\sup \left\{T(\phi): \phi \in C_{c}^{\infty}\left(\mathcal{D} ; \Lambda^{k} \mathbb{R}^{n}\right),\left\|\frac{\phi}{\rho}\right\|_{\infty} \leq 1\right\} .
$$

Here (and throughout this paper) $\rho$ is the function defined in (1.3).

I next define several seminorms on currents in $\dot{\mathcal{M}}_{1, \rho}$. The first, denoted $\mathbf{F}_{\rho}$, is a weighted analog of the flat norm from geometric measure theory and will be used chiefly for currents in the two-dimensional cross-section $\mathcal{D}_{0}$. For these it has a simple form, given in Lemma 4 . We will also need a more complicated seminorm $\mathbf{F}_{p, \rho, K}$. As the subscripts indicate, the definition depends on the functions $\rho$ and $p$ defined in (1.3) and (2.3) and on a compact $K \subset \mathcal{D}$. The usefulness of these norms is based largely on the following compactness lemma:

Lemma 2. If $\left\{R_{k}\right\} \subset \dot{\mathcal{M}}_{1, \rho}\left(\mathcal{D}_{0}\right)$ is a sequence of currents for which $\mathbf{M}_{\rho}\left(R_{k}\right) \leq C$, then there exists some $R \in \mathcal{M}_{1, \rho}\left(\mathcal{D}_{0}\right)$ such that, after passing to a subsequence,

$$
\mathbf{F}_{\rho}\left(R_{k}-R\right) \rightarrow 0 \quad \text { as } k \rightarrow \infty .
$$


And if $\left\{T_{k}\right\} \subset \dot{\mathcal{M}}_{1, \rho}(\mathcal{D})$ is a sequence of currents for which $\mathbf{M}_{\rho}\left(T_{k}\right) \leq C$, then for every compact $K \subset \mathcal{D}$ there exists some $T \in \dot{\mathcal{M}}_{1, \rho}$ such that after passing to a subsequence,

$$
\mathbf{F}_{p, \rho, K}\left(T_{k}-T\right) \rightarrow 0 \quad \text { as } k \rightarrow \infty
$$

The proof is deferred to Section 9; for now I only give the definitions. For a compact subset $K \subset \mathcal{D}$ and currents $T \in \dot{\mathcal{M}}_{1}(\mathcal{D})$, I define the flat norm

$$
\mathbf{F}_{K}(T)=\inf \left\{\mathbf{M}_{K}(S): S \in \mathcal{M}_{2}(\mathcal{D}), \partial S=T \text { in } K\right\}
$$

(This differs somewhat from the usual definition.) I also define, for $T \in \dot{\mathcal{M}}_{1}(\mathcal{D})$

$$
\mathbf{F}_{\rho}(T):=\inf \left\{\mathbf{M}_{\rho}(S): S \in \mathcal{M}_{2, \rho}(\mathcal{D}), \partial S=T \text { in } \mathcal{D}\right\}
$$

and

$$
\mathbf{F}_{p, \rho}(T):=\mathbf{F}_{\rho}\left(p_{\#} T\right)
$$

Observe that if $T_{X}$ is the current associated with integration along a Lipschitz curve $X$ as in (3.10) below, then $p_{\#} T_{X}$ is just the current $T_{p \circ X}$ associated with $p \circ X$, which is a Lipschitz curve in the two dimensional ellipse $\mathcal{D}_{0}$, indeed in the half-ellipse $\mathcal{D}_{0}{ }^{+}$. Finally I define, for a given compact $K \subset \mathcal{D}$

$$
\mathbf{F}_{p, \rho, K}(T)=\mathbf{F}_{p, \rho}(T)+\mathbf{F}_{K}(T)
$$

All facts stated about the $\mathbf{F}_{p, \rho, K}$ seminorm are valid as well for the $\mathbf{F}_{p, \rho}$ seminorm, which is just the $\mathbf{F}_{p, \rho, K}$ seminorm for $K=\emptyset$.

For the arguments in Section 4 on local minimizers of $E_{\Omega}$, it is natural to work with the $\mathbf{F}_{p, \rho}$ seminorm, which amounts to using the $\mathbf{F}_{\rho}$ norm (in the simple form given in Lemma 4 ) in the two-dimensional cross-section $\mathcal{D}_{0}$. A drawback of the $\mathbf{F}_{p, \rho}$ seminorm is that it is extremely degenerate: it is clear that $\mathbf{F}_{p, \rho}\left(T_{1}-T_{2}\right)=0$ whenever $p_{\#} T_{1}=p_{\#} T_{2}$. This degeneracy makes it hard to find isolated local minimizers of $E_{\Omega}$. The $\mathbf{F}_{p, \rho, K}$ seminorms are introduced because they are less degenerate, and therefore support isolated local minimizers.

\subsection{Representations of currents}

It is helpful to reformulate $E_{\Omega}$ as a functional on $\dot{\mathcal{R}}_{1, \rho}(\mathcal{D})$. If $X:(a, b) \rightarrow \mathcal{D}$ is a parametrized curve then one can define a current $T_{X} \in \mathcal{R}_{1}(\mathcal{D})$ corresponding to integration along $X$ :

$$
T_{X}\left(\phi^{i} \mathrm{~d} x^{i}\right)=\int_{a}^{b} \phi^{i}(X(t)) \dot{X}^{i}(t) \mathrm{d} t, \quad \quad \phi=\phi^{i} \mathrm{~d} x^{i} \in C_{c}^{\infty}\left(\mathcal{D} ; \Lambda^{1} \mathbb{R}^{3}\right) .
$$

In addition, $\partial T=0$ in $\mathcal{D}$ if and only if $X$ has no boundary in the sense of (1.7).

I will regard the functional $E_{\Omega}$ defined in (1.6) as a special case of a functional, still denoted $E_{\Omega}$, defined on currents $T \in \stackrel{\circ}{\mathcal{R}}_{1, \rho}(\mathcal{D})$ :

$$
E_{\Omega}(T):=\int \rho\|T\|(\mathrm{d} x)-\Omega T\left(\rho^{2} \mathrm{~d} x^{3}\right), \quad T \in \stackrel{\circ}{\mathcal{R}}_{1, \rho}(\mathcal{D})
$$

If $X$ is a parametrized curve without boundary in $\mathcal{D}$ and $T_{X} \in \stackrel{\circ}{\mathcal{R}}_{1}(\mathcal{D})$ is defined as in $(3.10)$ then $E_{\Omega}\left(T_{X}\right)=$ $E_{\Omega}(X)$, where the right-hand side is understood in the sense of (1.6). I will henceforth always take $E_{\Omega}$ to be as defined in (3.11). Note that the critical value $\Omega_{1}$ defined in (1.8) in terms of Lipschitz curves can be equivalently defined by

$$
\Omega_{1}:=\inf \left\{\Omega>0: \exists T \in \stackrel{\circ}{\mathcal{R}}_{1, \rho}(\mathcal{D}) \text { such that } E_{\Omega}(T)<0\right\} .
$$


I will also sometimes use the notation $E_{\Omega}=E_{0}-\Omega L$, where

$$
E_{0}(T)=\int \rho \mathrm{d}\|T\|=\mathbf{M}_{\rho}(T), \quad L(T)=T\left(\rho^{2} \mathrm{~d} x^{3}\right) .
$$

The following decomposition is useful:

Lemma 3. If $U \subset \mathbb{R}^{n}, T \in \mathcal{R}_{1}(U)$ and $\partial T$ has locally finite mass in $U$, then there exists a family of at most countably many Lipschitz curves $\left\{X_{i}\right\}$ such that $T=\sum_{i} T_{X_{i}}$, with

$$
\|T\|=\sum_{i}\left\|T_{X_{i}}\right\| \quad \text { and }\|\partial T\|=\sum_{i}\left\|\partial T_{X_{i}}\right\| \text { as measures. }
$$

In particular, if $T \in \dot{\mathcal{R}}_{1, \rho}(\mathcal{D})$ then there exist $X_{i}$ such that $T_{X_{i}} \in \dot{\mathcal{R}}_{1, \rho}(\mathcal{D}), T=\sum T_{X_{i}}$, and $E_{\Omega}(T)=$ $\sum_{i} E_{\Omega}\left(T_{X_{i}}\right)$. (Here $T_{X_{i}}$ is as in (3.10).)

The proof is given in Section 9.

I will later reduce the problem of finding $\mathbf{F}_{p, \rho, K}$-local minimizers of $E_{\Omega}$ in $\stackrel{\circ}{\mathcal{R}}_{1, \rho}(\mathcal{D})$ to the study of $\mathbf{F}_{\rho}$-local minimizers in $\mathcal{R}_{1, \rho}\left(\mathcal{D}_{0}\right)$. This is useful because in the 2-dimensional domain $\mathcal{D}_{0}$ one can work with BV functions instead of currents:

Lemma 4. If $T \in \stackrel{\circ}{\mathcal{R}}_{1, \rho}\left(\mathcal{D}_{0}\right)$ then there exists $u \in B V_{\text {loc }}\left(\mathcal{D}_{0} ; \mathbb{Z}\right)$ such that

$$
T(\phi)=\int_{\mathcal{D}_{0}} u \mathrm{~d} \phi=\int_{\mathcal{D}_{0}} \phi \wedge \mathrm{d} u \quad \text { for all } \phi \in C_{0}^{\infty}\left(\mathcal{D}_{0} ; \Lambda^{1} \mathbb{R}^{2}\right) .
$$

When this holds I will write $T=\star \mathrm{d} u$. In addition,

$$
\mathbf{F}_{\rho}(T)=\inf _{c \in \mathbb{R}} \int_{\mathcal{D}_{0}} \rho|u-c|, \quad E_{0}(T)=\int_{\mathcal{D}_{0}} \rho|\nabla u| .
$$

Conversely, given $u \in B V_{\text {loc }}\left(\mathcal{D}_{0} ; \mathbb{Z}\right)$ such that $\int_{\mathcal{D}_{0}} \rho|\nabla u|<\infty$, the current $T=\star \mathrm{d} u$ defined by (3.15) belongs to $\mathcal{R}_{1, \rho}\left(\mathcal{D}_{0}\right)$, and (3.16) holds. Finally, if $T$ is supported in $\mathcal{D}_{0}{ }^{+}$then $u$ can be taken to be supported in $\mathcal{D}_{0}{ }^{+}$, and when this is done, $\mathbf{F}_{\rho}(T)=\int_{\mathcal{D}_{0}} \rho|u|$.

Proof. For $T \in \stackrel{\circ}{\mathcal{R}}_{1, \rho}\left(\mathcal{D}_{0}\right)$, since $\partial T=0$, there exists an integer multiplicity locally normal 2-current $S$ on $\mathcal{D}_{0}$ such that $\partial S=T$. In general, $n$-dimensional locally normal currents in $n$-dimensional domains can be identified with functions of locally bounded variation, so that there exists some $u \in B V_{\text {loc }}\left(\mathcal{D}_{0}\right)$ such that $S(\psi)=\int_{\mathcal{D}_{0}} \psi u$ for all compactly supported 2-forms $\psi$, and hence (3.15) holds. Since $S$ is integer multiplicity, $u$ is integer-valued.

The claim that $\mathbf{F}_{\rho}(T)=\inf _{c \in \mathbb{R}} \int_{\mathcal{D}_{0}} \rho|u-c|$ follows from two observations. First, if $S$ is a 2-current in $\mathcal{D}_{0}$ represented by integration against a function $v$, then $\mathbf{M}_{\rho}(S)=\int_{\mathcal{D}_{0}} \rho|v|$ by definition. Second, if $\partial S=\partial S^{\prime}=T$, then $\partial\left(S-S^{\prime}\right)=0$ in $\mathcal{D}_{0}$, and so the current $S-S^{\prime}$ is represented by integration against a constant function $c$.

The identity $E_{0}(T)=\int_{\mathcal{D}_{0}} \rho|\nabla u|$ is a direct consequence of the definitions. Conversely, if $u \in B V_{\text {loc }}\left(\mathcal{D}_{0} ; \mathbb{Z}\right)$ with $\int \rho|\nabla u|<\infty$, then it follows from standard properties of BV functions ${ }^{2}$ that $\star \mathrm{d} u \in \stackrel{\circ}{\mathcal{R}}_{1, \rho}\left(\mathcal{D}_{0} ; \mathbb{Z}\right)$ and (3.16) holds.

If $T$ is supported in $\mathcal{D}_{0}{ }^{+}$and (3.15) holds, then $\nabla u=0$ in the sense of distributions in $\mathcal{D}_{0} \backslash \mathcal{D}_{0}{ }^{+}$. Thus $u=c$ almost everywhere in this set, and so by adding a constant we can arrange that the support of $u$ is contained in $\mathcal{D}_{0}{ }^{+}$. Finally, if supp $u \subset \mathcal{D}_{0}{ }^{+}$and $c^{\prime}$ is any constant, then $|u|-\left|u-c^{\prime}\right|=-\left|c^{\prime}\right|$ a.e. in $\mathcal{D}_{0} \backslash \mathcal{D}_{0}{ }^{+}$, and $|u|-\left|u-c^{\prime}\right| \leq\left|c^{\prime}\right|$ in $\mathcal{D}_{0}{ }^{+}$, and so

$$
\int_{\mathcal{D}_{0}} \rho\left(|u|-\left|u-c^{\prime}\right|\right) \leq 0
$$

\footnotetext{
${ }^{2}$ That is, the fact that the gradient of a function in $B V_{\text {loc }}$ is carried by an $(n-1)$ rectifiable set.
} 
for every $c^{\prime}$. Thus $\int_{\mathcal{D}_{0}} \rho|u|=\inf _{c^{\prime} \in \mathbb{R}} \int_{\mathcal{D}_{0}} \rho\left|u-c^{\prime}\right|=\mathbf{F}_{\rho}(T)$.

The following lemma shows that $L$ is continuous with respect to the $\mathbf{F}_{p, \rho, K}$ seminorms.

Lemma 5. There exists a constant $C$ such that for any current $T \in \dot{\mathcal{M}}_{1, \rho}(\mathcal{D})$

$$
|L(T)|=\left|T\left(\rho^{2} \mathrm{~d} x^{3}\right)\right| \leq C \mathbf{F}_{p, \rho}(T) .
$$

As a result, $|L(T)| \leq \mathbf{F}_{p, \rho, K}(T)$ for all compact $K \subset \mathcal{D}$.

Proof. First, one easily checks from the definitions $(2.3)$, (1.3) of $p$ and $\rho$ that $p^{\#}\left(\rho^{2} \mathrm{~d} x^{3}\right)=\rho^{2} \mathrm{~d} x^{3}$, and so $T\left(\rho^{2} \mathrm{~d} x^{3}\right)=p_{\#} T\left(\rho^{2} \mathrm{~d} x^{3}\right)$. Also, by definition, $\mathbf{F}_{p, \rho}(T)=\mathbf{F}_{\rho}\left(p_{\#} T\right)$, so to prove (3.17), it suffices to check that

$$
\left|T\left(\rho^{2} \mathrm{~d} x^{3}\right)\right| \leq C \mathbf{F}_{\rho}(T)
$$

for all $T \in \dot{\mathcal{M}}_{1, \rho}\left(\mathcal{D}_{0}\right)$ with support in $\mathcal{D}_{0}{ }^{+}$. Given such a current $T$, write $T=\star \mathrm{d} u$ and using Lemma 4 ,

$$
T\left(\rho^{2} \mathrm{~d} x^{3}\right)=\int_{\mathcal{D}_{0}} 2 \rho \rho_{x_{2}} \mathrm{~d} x^{2} \wedge \mathrm{d} x^{3} \leq C \int_{\mathcal{D}_{0}} \rho|u|=\mathbf{F}_{\rho}(T) .
$$

\section{LOCAL MINIMIZERS FOR THE REDUCED ENERGY}

The main result of this section provides a description of large numbers of nontrivial local minimizers of the line energy $E_{\Omega}$. The proof relies heavily on earlier joint work with A. Aftalion, including [1], which shows among other results that the energy of a vortex filament in $\mathcal{D}$ can be lowered by pushing it forward via the map $p$, see (2.3), into the two-dimensional cross-section $\mathcal{D}_{0}$ of the ellipsoid $\mathcal{D}$; and [2], which constructs certain constrained minimizers of $E_{\Omega}$ in the space of Lipschitz curves in $\mathcal{D}_{0}$. The relevant results are described more precisely below. The point here is to show that these constrained minimizers in $\mathcal{D}_{0}$ can be used to construct large families of local (but unconstrained) minimizers of $E_{\Omega}$ in spaces of integral currents on $\mathcal{D}$, with respect to the norms on these spaces introduced in Section 3.

First we give:

Definition 1. For compact $K \subset \mathcal{D}$, a set $M \subset \stackrel{\circ}{\mathcal{R}}_{1, \rho}(\mathcal{D})$ is said to be a $\mathbf{F}_{p, \rho, K}$-local minimizing set of $E_{\Omega}$ if $M$ is compact with respect to the $\mathbf{F}_{p, \rho, K}$ seminorm, and if there exists $\sigma>0$ such that for

$$
O_{\sigma}:=\left\{T \in \stackrel{\circ}{\mathcal{R}}_{1, \rho}(\mathcal{D}): \min _{S \in M} \mathbf{F}_{p, \rho, K}(T-S)<\sigma\right\},
$$

the functional $E_{\Omega}$ attains its minimum in $O_{\sigma}$, and in addition, for $T \in O_{\sigma}$,

$$
E_{\Omega}(T)=\min _{S \in O_{\sigma}} E_{\Omega}(S) \text { if and only if } T \in M
$$

A current $T \in \stackrel{\circ}{\mathcal{R}}_{1, \rho}(\mathcal{D})$ is said to be a $\mathbf{F}_{p, \rho, K}$-local minimizer of $E_{\Omega}$ if $M=\{T\}$ is a $\mathbf{F}_{p, \rho, K}$-local minimizing set.

A $\mathbf{F}_{\rho}$-local minimizing set is defined in a strictly analogous way.

It is clear that if $K_{1} \subset K_{2}$ are two compact sets, then any $\mathbf{F}_{p, \rho, K_{1}}$-local minimizing set is also a $\mathbf{F}_{p, \rho, K_{2}}$-local minimizing set.

In the statement of the theorem below, $a_{1}, a_{2}$ refer to parameters in the definitions of $\rho, \mathcal{D}$; the condition $a_{1}=a_{2}$ means that $\mathcal{D}$ is rotationally symmetric about the $x_{3}$ axis, and when this holds, the symmetry leads to a large number of local minimizers. Recall that $\Omega_{1}$ is defined in (3.12) and that we have assumed for concreteness that $0<a_{1} \leq a_{2}$. 
Theorem 1. There exists $\Omega_{0}<\Omega_{1}$ such that for every $\Omega>\Omega_{0}$, there exists a set $M_{\Omega} \subset \stackrel{\circ}{\mathcal{R}}_{1, \rho}(\mathcal{D})$ of multiplicity 1 currents, compact with respect to the $\mathbf{F}_{p, \rho}$ seminorm, such that for every positive integer $k$,

$$
M_{\Omega}^{k}:=\left\{\sum_{i=1}^{k} T_{i}: T_{i} \in M_{\Omega}\right\}
$$

is a $\mathbf{F}_{p, \rho, K}$-local minimizing set. Moreover, if $a_{1}<a_{2}$ then for a.e. $\Omega>\Omega_{0}, M_{\Omega}$ contains exactly one element, denoted $T_{\Omega}^{+}$, supported in $\left\{x \in \mathcal{D}_{0}: x_{2} \geq 0\right\}$, and exactly one element $T_{\Omega}^{-}$supported in $\left\{x \in \mathcal{D}_{0}: x_{2} \leq 0\right\}$, and nothing else. Either $T_{\Omega}^{+}$and $T_{\Omega}^{-}$have disjoint support, or they are equal and supported on the $x_{3}$-axis. In the latter case, $M_{\Omega}^{k}$ consists of a single $\mathbf{F}_{p, \rho, K}$-local minimizer.

One can think of $M_{\Omega}^{k}$ as the set of local minimizers that have $k$ vortex lines (generally not all distinct). Note that the sum on the right-hand side of (4.2) may include terms repeated according to multiplicity.

In physical terms, the fact that $\Omega_{0}<\Omega_{1}$ shows the existence of stable vortex filaments in some parameter range where the energy of a vortex line is necessarily positive.

The above theorem is true for any compact $K \subset \mathcal{D}$, and in particular for the highly degenerate $\mathbf{F}_{p, \rho}$ seminorm, corresponding to $K=\emptyset$. If $K$ is chosen more carefully we generically obtain isolated local minimizers rather than locally minimizing sets.

Corollary 1. For $a_{1}<a_{2}$ and for a.e. $\Omega>\Omega_{0}$, for every pair of nonnegative integers $k_{1}$, $k_{2}$, there exists a compact set $K \subset \mathcal{D}$ such that $T_{\Omega ; k_{1}, k_{2}}=k_{1} T_{\Omega}^{+}+k_{2} T_{\Omega}^{-}$is a $\mathbf{F}_{p, \rho, K}$-local minimizer for $E_{\Omega}$.

Proof. For $a_{1}<a_{2}$ and a.e. $\Omega>\Omega_{0}, M_{\Omega}$ contains either one or two elements. In the former case, the conclusion follows directly from the definition of local minimizer, so we assume that $M_{\Omega}$ contains exactly two distinct currents $T_{\Omega}^{+} \neq T_{\Omega}^{-}$. Then $M_{\Omega}^{k}=\left\{T_{\Omega ; k_{1}, k_{2}}: k_{1}+k_{2}=k, k_{1}, k_{2} \geq 0\right\}$ is a finite set. As a result, if the compact set $K$ is sufficiently large and $\sigma$ is sufficiently small, then

$$
\mathbf{F}_{K}\left(T_{\Omega ; k_{1}, k_{2}}-T_{\Omega ; l_{1}, l_{2}}\right)>2 \sigma
$$

whenever $k_{1}+k_{2}=l_{1}+l_{2}=k$ and $k_{1} \neq l_{1}$. Consequently $O_{\Omega, \sigma}^{k}$ (defined as in (4.1), with $M$ replaced by $M_{\Omega}^{k}$ ) consists of $k+1$ pairwise disjoint components, each of the form

$$
\left\{T \in \stackrel{\circ}{\mathcal{R}}_{1, \rho}(\mathcal{D}): \mathbf{F}_{p, \rho, K}\left(T-T_{\Omega ; k_{1}, k_{2}}\right)<\sigma\right\}
$$

and from this and the previous theorem, it is easy to see that each $T_{\Omega ; k_{1}, k_{2}}$ is a $\mathbf{F}_{p, \rho, K^{-}}$-local minimizer.

I will prove Theorem 1 by first proving an analogous result for a class of currents in the two-dimensional ellipse $\mathcal{D}_{0}$, and then showing that the theorem reduces to this special situation. The key point in this reduction is supplied by the following lemma, which shows that a general current in $\mathcal{D}$ can be pushed forward into $\mathcal{D}_{0}$ in a way that reduces its energy.

Lemma 6. If $T \in \mathcal{R}_{1, \rho}(\mathcal{D})$, then (for $p: \mathcal{D} \rightarrow \mathcal{D}_{0}$ as defined in (2.3)),

$$
L\left(p_{\#} T\right)=L(T) \quad \text { and } \quad E_{0}\left(p_{\#} T\right) \leq E_{0}(T) .
$$

If equality holds in the latter then $T$ can be written in the form $T=\sum T_{X_{i}}$ as in (3.14), where each $T_{X_{i}}$ satisfies:

$$
\begin{aligned}
& \quad \text { if } a_{1}<a_{2}: \quad \operatorname{supp} T_{X_{i}} \subset \mathcal{D}_{0} ; \text { or } \vec{T}_{X_{i}}(x)= \pm e_{3} \text { for }\left\|T_{X_{i}}\right\| \text { a.e. } x \text {. } \\
& \text { if } a_{1}=a_{2}: \quad \text { after a rotation about the } x_{3} \text { axis, supp } T_{X_{i}} \subset\left\{x: x_{1}=0\right\} \text {. }
\end{aligned}
$$


Recall that if $T_{X}$ is a current associated with integration along a Lipschitz curve $X: \mathbb{R} \supset[a, b] \rightarrow \mathcal{D}$, then $p_{\#} T_{X}=T_{p \circ X}$.

The proof is given in Section 9. The point is that it reduces, via Lemma 3, to the case of a current of the form $T_{X}$, where $X$ is a Lipschitz curve. In this case the lemma is easy and has already been proved in [1], apart from the conditions for equality, which are not hard to deduce.

The next lemma, whose proof also appears in Section 9, is similarly proved by combining analogous results from [1] in the context of Lipschitz curves with Lemma 3.

Lemma 7. There exists a constant $C>0$ such that

$$
|L(T)| \leq C E_{0}(T)^{3 / 2}
$$

for every $T \in \stackrel{\circ}{\mathcal{R}}_{1, \rho}(\mathcal{D})$.

Theorem 1 will be deduced from the following proposition. I use the notation

$$
\stackrel{\circ}{\mathcal{R}}_{1, \rho}^{*}:=\left\{T \in \stackrel{\circ}{\mathcal{R}}_{1, \rho}\left(\mathcal{D}_{0}\right): \operatorname{supp}(T) \subset \mathcal{D}_{0}{ }^{+}\right\} .
$$

Proposition 1. There exists $\Omega_{0}<\Omega_{1}$ such that for every $\Omega>\Omega_{0}$, there exists a set $M_{\Omega}^{*} \subset \stackrel{\circ}{\mathcal{R}}_{1, \rho}^{*}$ of multiplicity 1 currents such that for every positive integer $k$

$$
M_{\Omega}^{*, k}:=\left\{\sum_{i=1}^{k} T_{i}: T_{i} \in M_{\Omega}^{*}\right\}
$$

is a $\mathbf{F}_{\rho}$-local minimizing set in $\stackrel{\circ}{\mathcal{R}}_{1, \rho}\left(\mathcal{D}_{0}\right)$. Moreover for almost every $\Omega>\Omega_{0}, M_{\Omega}^{*}$ contains exactly one element, and its support is either the $x_{3}$ axis or is bounded away from the $x_{3}$ axis.

Proof. I will first show that the conclusions of the proposition hold for $\Omega>\Omega_{1}$ as defined in (3.12). In the final step I will show how to modify the argument to obtain the same conclusions for $\Omega>\Omega_{0}$, for $\Omega_{0}<\Omega_{1}$ as defined in (4.16).

A couple of times during the proof I will need the elementary facts that, if $u^{ \pm}, v^{ \pm}$denote the positive and negative parts of functions $u, v \in B V_{\text {loc }}$ then

$$
\begin{aligned}
|u-v| & =\left|u^{+}-v^{+}\right|+\left|u^{-}-v^{-}\right|, \\
|\nabla u| & =\left|\nabla u^{+}\right|+\left|\nabla u^{-}\right| \text {as measures. }
\end{aligned}
$$

1. In view of Lemma 4 , there is a one-to-one correspondence between currents $T \in \stackrel{\circ}{\mathcal{R}}_{1, \rho}\left(\mathcal{D}_{0}\right)$ with support in $\mathcal{D}_{0}{ }^{+}$and functions $u \in B V_{\text {loc }}\left(\mathcal{D}_{0} ; \mathbb{Z}\right)$ supported in $\mathcal{D}_{0}{ }^{+}$and satisfying $\int_{\mathcal{D}_{0}} \rho|\nabla u|<\infty$. Throughout this proof I will work with $B V_{\text {loc }}$ functions supported in $\mathcal{D}_{0}{ }^{+}$, so that $\mathbf{F}_{\rho}(T)=\int_{\mathcal{D}_{0}} \rho|u|$ when $T=\star \mathrm{d} u$.

First note that if $T=\star \mathrm{d} u$ as in (3.15), then

$$
E_{\Omega}(T)=\int_{\mathcal{D}_{0}} \rho|\nabla u|-2 \Omega \int_{\mathcal{D}_{0}} \rho \rho_{x_{2}} u \mathrm{~d} x_{2} \mathrm{~d} x_{3}=: E_{\Omega}{ }^{*}(u) .
$$

Note also that if $u \in B V_{\text {loc }}\left(\mathcal{D}_{0} ; \mathbb{Z}\right), u \geq 0$, and $\operatorname{supp} u \subset \mathcal{D}_{0}{ }^{+}$then $E_{\Omega}{ }^{*}(u) \geq 0$, as $\rho \rho_{x_{2}}<0$ in $\mathcal{D}_{0}{ }^{+}$. For the same reason, $E_{0}(\star \mathrm{d} u)=\mathbf{M}_{\rho}(\star \mathrm{d} u)$ is bounded for any $E_{\Omega}$-minimizing sequence in

$$
\mathcal{A}:=\left\{u \in B V_{\mathrm{loc}}\left(\mathcal{D}_{0} ; \mathbb{Z}\right): \operatorname{supp} u \subset \mathcal{D}_{0}{ }^{+}, u \geq-1 \text { a.e. }\right\}
$$

It therefore follows from a standard lowersemicontinuity argument using the compactness Lemma 2 that $E_{\Omega}{ }^{*}$ attains its minimum in $\mathcal{A}$. A proof with more details can be found in [2], Proposition 1.1, where it is also shown 
that the support of any minimizer is either compactly contained in $\left\{x \in \mathcal{D}_{0}: x_{2}>0\right\}$ or else is all of $\mathcal{D}_{0}{ }^{+}$. (In the latter case, the associated current $\star \mathrm{d} u$ is supported on the $x_{3}$ axis.) It is clear that any minimizing function is nonpositive for a.e. $x$. Moreover, the definition (3.12) of $\Omega_{1}$ implies that $\inf _{v \in \mathcal{A}} E_{\Omega}{ }^{*}(v)<0$ for $\Omega>\Omega_{1}$, and so for such $\Omega$ any minimizer is nontrivial. Define

$$
M_{\Omega}^{*}:=\left\{\star \mathrm{d} u: u \in \mathcal{A}, E_{\Omega}^{*}(u)=\min _{v \in \mathcal{A}} E_{\Omega}^{*}(v)\right\}
$$

and let $M_{\Omega}^{*, k}$ be as in (4.5). It follows from [2], Theorem 1.3 that for a.e. $\Omega, M_{\Omega}^{*}$ contains exactly one element. Furthermore, the same compactness and lowersemicontinuity argument that proves existence of minimizers of $E_{\Omega}{ }^{*}$ in $\mathcal{A}$ also shows that the set $M_{\Omega}^{*}$ of such minimizers is $\mathbf{F}_{\rho}$-compact, and the $\mathbf{F}_{\rho}$-compactness of $M_{\Omega}^{*, k}$ for arbitrary $k$ follows easily.

2. Now fix $k \geq 1$ and $T=\star \mathrm{d} v \in O_{\Omega, \sigma}^{*, k}$, where

$$
O_{\Omega, \sigma}^{*, k}:=\left\{T \in \stackrel{\circ}{\mathcal{R}}_{1, \rho}\left(\mathcal{D}_{0}\right): \min _{S \in M_{\Omega}^{*, k}} \mathbf{F}_{\rho}(T-S)<\sigma\right\}
$$

I will show that if $\sigma$ is taken to be sufficiently small, then $v$ can be written in the form $v=v_{0}+\cdots+v_{k}$, with

$$
E_{\Omega}{ }^{*}(v)=\sum_{j=0}^{k} E_{\Omega}{ }^{*}\left(v_{j}\right), \quad v_{j} \in \mathcal{A} \text { for } j=1 \ldots, k, \quad \text { and } E_{\Omega}{ }^{*}\left(v_{0}\right) \geq 0
$$

and moreover $E_{\Omega}\left(v_{0}\right)>0$ if $v_{0} \neq 0$. This will prove that $M_{\Omega}^{*, k}$ is $\mathbf{F}_{\rho}$-local minimizing, because (4.11) implies that

$$
E_{\Omega}{ }^{*}(v) \geq \sum_{j=1}^{k} E_{\Omega}{ }^{*}\left(v_{j}\right) \geq k \min _{w \in \mathcal{A}} E_{\Omega}{ }^{*}(w)
$$

with equality iff $v_{0}=0$ and $\star \mathrm{d} v_{j} \in M_{\Omega}^{*}$ for $j=1 \ldots, k$, in other words, if and only if $v \in M_{\Omega}^{*, k}$.

3. To decompose $v$, first define $v^{\prime}=\max \{v,-k\}=(v+k)^{+}-k$ and $v_{0}=v-v^{\prime}=-(v+k)^{-}$. Then clearly $v=v_{0}+v^{\prime}$, and $v^{\prime} \geq-k$ almost everywhere, and in addition $|\nabla v|=\left|\nabla v_{0}\right|+\left|\nabla v^{\prime}\right|$ as measures (by (4.7) applied to $v+k$ ), which implies that

$$
E_{\Omega}^{*}(v)=E_{\Omega}^{*}\left(v_{0}\right)+E_{\Omega}^{*}\left(v^{\prime}\right)
$$

I claim that $E_{\Omega}{ }^{*}\left(v_{0}\right) \geq 0$, with strict inequality unless $v_{0}=0$. To prove this, note that by the definition (4.10) of $O_{\Omega, \sigma}^{*, k}$, there exists some $S=\star \mathrm{d} u \in M_{\Omega}^{*, k}$ such that

$$
\int \rho|u-v|=\mathbf{F}_{\rho}(\star \mathrm{d} u-\star \mathrm{d} v)<\sigma .
$$

By the definition of $M_{\Omega}^{*, k}, u \geq-k$ a.e., so (4.6) applied to $v+k, u+k$ implies that $\left|v_{0}\right| \leq\left|v_{0}\right|+\left|u-v^{\prime}\right|=|u-v|$. Thus

$$
\left|L\left(\star \mathrm{d} v_{0}\right)\right|=\left|\int_{\mathcal{D}_{0}} 2 \rho \rho_{x_{2}} v_{0}\right| \leq C \int_{\mathcal{D}_{0}} \rho\left|v_{0}\right| \leq C \int_{\mathcal{D}_{0}} \rho|u-v|<C \sigma .
$$

It follows from this and Lemma 7 that

$$
E_{\Omega}\left(\star \mathrm{d} v_{0}\right)=E_{0}\left(\star \mathrm{d} v_{0}\right)-\Omega L\left(\star \mathrm{d} v_{0}\right) \geq c\left|L\left(\star \mathrm{d} v_{0}\right)\right|^{2 / 3}-\Omega L\left(\star \mathrm{d} v_{0}\right)>0
$$

for a suitably small choice of $\sigma$.

4. In view of (4.13), it now suffices to show that $v^{\prime}$ can be written as a sum $v^{\prime}=v_{1}+\ldots+v_{k}$ with $v_{j} \in \mathcal{A}$ for all $j=1, \ldots, k$ and $E_{\Omega}{ }^{*}\left(v^{\prime}\right)=\sum_{j=1}^{k} E_{\Omega}{ }^{*}\left(v_{j}\right)$. In fact, by an induction argument, it is enough to show that we can write $v^{\prime}=v_{1}+v^{\prime \prime}$, where $v_{1} \in \mathcal{A}, v^{\prime \prime} \geq-k+1$ a.e., and $E_{\Omega}{ }^{*}\left(v^{\prime}\right)=E_{\Omega}{ }^{*}\left(v_{1}\right)+E_{\Omega}{ }^{*}\left(v^{\prime \prime}\right)$. To achieve this, 
simply let $v^{\prime \prime}=\max \left\{-k+1, v^{\prime}\right\}$ and $v_{1}=v^{\prime}-v^{\prime \prime}$; the verification that $E_{\Omega}{ }^{*}\left(v^{\prime}\right)=E_{\Omega}{ }^{*}\left(v_{1}\right)+E_{\Omega}{ }^{*}\left(v^{\prime \prime}\right)$ is exactly like the proof of (4.13) above.

This completes the proof of the proposition for $\Omega>\Omega_{1}$.

5. I now show that for sufficiently large there is a number $\Omega_{0}<\Omega_{1}$ such that the conclusions of the proposition remain valid for all $\Omega>\Omega_{0}$.

Define $L^{*}(u)=L(\star \mathrm{d} u)=2 \int_{\mathcal{D}_{0}} \rho \rho_{x_{2}} u$, and, for $0<\ell<\ell_{\max }:=\max _{u \in \mathcal{A}} L^{*}(u)$, define

$$
\begin{aligned}
\mathcal{A}_{\ell} & :=\left\{u \in \mathcal{A}: L^{*}(u)=\ell\right\}, \\
\mathcal{A}_{>\ell} & :=\left\{u \in \mathcal{A}: L^{*}(u)>\ell\right\} \\
\mathcal{A}_{\geq \ell} & :=\left\{u \in \mathcal{A}: L^{*}(u) \geq \ell\right\}
\end{aligned}
$$

and

and finally

$$
e_{0}^{*}(\ell):=\min \left\{E_{0}^{*}(u): u \in \mathcal{A}_{\ell}\right\}
$$

$$
\Omega_{0}:=\inf _{\ell^{\prime}>\ell} \frac{e_{0}^{*}\left(\ell^{\prime}\right)-e_{0}^{*}(\ell)}{\ell^{\prime}-\ell}
$$

The definition of $\Omega_{1}$ can be rewritten as $\Omega_{1}=\inf _{\ell^{\prime}>0} \frac{e_{0}^{*}\left(\ell^{\prime}\right)}{\ell^{\prime}}=\inf _{\ell^{\prime}>0} \frac{e_{0}^{*}\left(\ell^{\prime}\right)-e_{0}^{*}(0)}{\ell^{\prime}-0}$. Lemma 7 implies that for all $\ell$ sufficiently close to $0, e_{0}^{*}(\ell) \geq c \ell^{2 / 3}$, and it follows that $\Omega_{0}<\Omega_{1}$.

Now fix $\Omega>\Omega_{0}$ and $\ell<\ell^{\prime}$ such that

$$
\Omega\left(\ell^{\prime}-\ell\right)>e_{0}^{*}\left(\ell^{\prime}\right)-e_{0}^{*}(\ell)
$$

Since $\mathcal{A}_{\geq \ell}$ is $\mathbf{F}_{\rho}$-closed, by Lemma 5 , familiar compactness and lowersemicontinuity arguments yield at least one minimizer of $E_{\Omega}$ in $\mathcal{A}_{\geq \ell}$. Define

$$
M_{\Omega}^{*}:=\left\{\star \mathrm{d} u: u \in \mathcal{A}_{\geq \ell}, E_{\Omega}{ }^{*}(u)=\min _{v \in \mathcal{A}_{\geq \ell}} E_{\Omega}^{*}(v)\right\} .
$$

Note that if $u \in M_{\Omega}^{*}$ then

$$
E_{\Omega}^{*}(u) \leq \inf _{v \in \mathcal{A}_{\ell^{\prime}}} E_{\Omega}^{*}(v)=e_{0}^{*}\left(\ell^{\prime}\right)-\Omega \ell^{\prime}<e_{0}^{*}(\ell)-\Omega \ell=\inf _{v \in \mathcal{A}_{\ell}} E_{\Omega}^{*}(v)
$$

using the definition of $e_{0}^{*}$ and (4.17). Thus in fact $\left\{u: \star \mathrm{d} u \in M_{\Omega}^{*}\right\} \subset \mathcal{A}_{>\ell}$. Note also that every minimizer is nontrivial, since $0 \notin \mathcal{A}_{>\ell}$.

Now define $M_{\Omega}^{*, k}$ and $O_{\Omega, \sigma}^{*, k}$. Given $v$ such that $\star \mathrm{d} v \in O_{\Omega, \sigma}^{*, k}$ and $\star \mathrm{d} u \in M_{\Omega}^{*, k}$ such that $\mathbf{F}_{\rho}(\star \mathrm{d} u-\star \mathrm{d} v)<\sigma$, it follows exactly as in step 3 above that if $\sigma$ is sufficiently small then $v=v_{0}+v^{\prime}$, where $v^{\prime} \geq-k$ almost everywhere, and $E_{\Omega}(v)=E_{\Omega}\left(v_{0}\right)+E_{\Omega}\left(v^{\prime}\right) \geq E_{\Omega}\left(v^{\prime}\right)$ with strict inequality unless $v_{0}=0$.

As in step 4, define $v^{\prime \prime}=\max \left\{v^{\prime}, k-1\right\}$ and $v_{1}=v^{\prime}-v^{\prime \prime}=-\left(v^{\prime}+k-1\right)^{-}$. Also define $u_{1}=-(u+k-1)^{-}$ for $u \in M_{\Omega}^{*, k}$ satisfying (4.14). The proof ${ }^{3}$ of Theorem 1.2, [1], shows that if $u, \tilde{u} \in M_{\Omega}^{*}$ then either $u \leq \tilde{u}$ or $\tilde{u} \leq u$ almost everywhere, and it follows that $u_{1} \in M_{\Omega}^{*}$. In addition, (4.6) implies that

$$
\int \rho\left|u_{1}-v_{1}\right| \leq \int \rho|u-v|<\sigma .
$$

As a result,

$$
L^{*}\left(v_{1}\right)=L^{*}\left(u_{1}\right)+L^{*}\left(v_{1}-u_{1}\right) \geq L^{*}\left(u_{1}\right)-C \sigma
$$

\footnotetext{
${ }^{3}$ The theorem cited is proved for $M_{\Omega}^{*}$ as defined in (4.9), but the proof works without change for $M_{\Omega}^{*}$ as defined in (4.18).
} 
as in (4.15). The compactness of $M_{\Omega}^{*}$ implies that there exists $\sigma_{0}>0$ such that $L^{*}\left(u_{1}\right)>\ell+\sigma_{0}$ for $u_{1} \in M_{\Omega}^{*}$. It follows that $v_{1} \in \mathcal{A}_{>\ell}$ if $\sigma$ is sufficiently small. The remainder of the proof follows as in step 4 above. (The results cited in step 1 above from [2] remain valid for $\Omega \in\left(\Omega_{0}, \Omega_{1}\right]$.)

Theorem 1 is an easy consequence of Proposition 1:

Proof of Theorem 1. 1. Fix $\Omega>\Omega_{0}$ and define $M_{\Omega}^{*}$ as in (4.18). Let $m^{*}=E_{\Omega}^{*}(u)$ for $\star \mathrm{d} u \in M_{\Omega}^{*}$. Define

$$
M_{\Omega}:=\left\{T \in \dot{\mathcal{R}}_{1, \rho}(\mathcal{D}): p_{\#} T \in M_{\Omega}^{*}, E_{\Omega}\left(p_{\#} T\right)=E_{\Omega}(T)=m^{*}\right\} .
$$

Given $T \in M_{\Omega}^{k}$, write $T=T_{1}+\cdots T_{k}$, with $T_{j} \in M_{\Omega}$ for all $j$. Then using the triangle inequality, the definition of $M_{\Omega}$, and the case of equality in (4.12),

$$
E_{\Omega}(T) \leq \sum E_{\Omega}\left(T_{i}\right)=\sum E_{\Omega}\left(p_{\#} T_{i}\right)=E_{\Omega}\left(\sum p_{\#} T_{i}\right)=E_{\Omega}\left(p_{\#} T\right)
$$

Comparing this with Lemma 6 , we find that $E_{\Omega}(T)=E_{\Omega}\left(p_{\#} T\right)$. It follows that the set $M_{\Omega}^{k}$ defined in (4.2) can be written

$$
M_{\Omega}^{k}=\left\{T \in \dot{\mathcal{R}}_{1, \rho}(\mathcal{D}): p_{\#} T \in M_{\Omega}^{*, k}, E_{\Omega}\left(p_{\#} T\right)=E_{\Omega}(T)=k m^{*}\right\} .
$$

Define $O_{\Omega, \sigma}^{k}:=\left\{T \in \stackrel{\circ}{\mathcal{R}}_{1, \rho}(\mathcal{D}): \min _{S \in M_{\Omega}^{k}} \mathbf{F}_{p, \rho, K}(T-S)<\sigma\right\}$. The definition (3.8) of the $\mathbf{F}_{p, \rho}$ seminorm implies that $T \in O_{\Omega, \sigma}^{k}$ if and only if $p_{\#} T \in O_{\Omega, \sigma}^{*, k}$. Thus if $T \in O_{\Omega, \sigma}^{k}$, then

$$
E_{\Omega}(T) \geq E_{\Omega}\left(p_{\#} T\right) \geq k m^{*},
$$

and at least one of the inequalities is strict unless $T \in M_{\Omega}^{k}$. So $M_{\Omega}^{k}$ is $\mathbf{F}_{p, \rho}=\mathbf{F}_{p, \rho, \emptyset}$ local minimizing, and hence also a local minimizing set with respect to the (stronger) $\mathbf{F}_{p, \rho, K}$ seminorm for any nonempty compact $K \subset \mathcal{D}$.

2. Recall that for almost every $\Omega>\Omega_{0}, M_{\Omega}^{*}$ contains a unique element, and that the support of this current is either the $x_{3}$ axis or is bounded away from the $x_{3}$ axis. Identifying $\dot{\mathcal{R}}_{1, \rho}\left(\mathcal{D}_{0}\right)$ as a subset of $\dot{\mathcal{R}}_{1, \rho}(\mathcal{D})$ in the natural way, this implies that there is a unique current $T_{\Omega}^{+} \in M_{\Omega}$ with support in $\mathcal{D}_{0}{ }^{+} \subset \mathcal{D}$. When $a_{1}<a_{2}$, the conditions for equality in Lemma 6 imply that any other element of $M_{\Omega}$ must have its support in $\mathcal{D}_{0}{ }^{-}$. But the problem of finding minimizers of $E_{\Omega}$ with support in $\mathcal{D}_{0}{ }^{-}$is identical to the minimization problem for currents supported in $\mathcal{D}_{0}{ }^{+}$, and so there is a unique $T_{\Omega}^{-} \in M_{\Omega}$ with support in $\mathcal{D}_{0}{ }^{-}$.

\section{The Jacobian and the Ginzburg-Landau energy}

This section collects some results that make precise, in various ways, the principle that the scaled GinzburgLandau energy $\frac{1}{\ln \varepsilon} e_{\varepsilon}(u)$ defined in (2.5) controls the Jacobian $J u$. The first results are refinements of estimates from [12]. The 3-dimensional version of this refined estimate (see Lem. 9) is vital for the proof of Theorem 2.

Lemma 8. For any $\lambda>1$ there exist $C, \alpha>0$ (depending only on $\lambda$ ) such that for any open set $U \subset \mathbb{R}^{2}$ and $u \in H^{1}\left(U ; \mathbb{R}^{2}\right)$,

$$
\left|\int_{U} \phi J u\right| \leq \lambda \int|\phi| \frac{e_{\varepsilon}(u)}{|\ln \varepsilon|}+C \varepsilon^{\alpha}\left(1+\|\phi\|_{W^{1, \infty}}\right)\left(\|\phi\|_{\infty}+1+\int_{\operatorname{supp} \phi}(|\phi|+1) e_{\varepsilon}(u) \mathrm{d} x\right)
$$

for all 0 -forms (i.e. functions) $\phi \in C_{c}^{0,1}(U)$.

Proof. 1. In [12], Theorem 2.1, it is proved that given $\lambda>1$ there exist $C, \alpha>0$ (depending on $\lambda$ ) such that for any $u, \phi$ as above,

$$
\left|\int_{U} \phi J u\right| \leq \lambda\|\phi\|_{\infty} \int_{\operatorname{supp} \phi} \frac{e_{\varepsilon}(u)}{|\ln \varepsilon|}+C \varepsilon^{\alpha}\|\phi\|_{C^{0,1}}\left(1+\int_{\operatorname{supp} \phi} e_{\varepsilon}(u)\right)\left(1+\mathcal{L}^{2}(\operatorname{supp} \phi)\right) .
$$


I will deduce the lemma from this estimate. The constants $\alpha$ in (5.1) and in (5.2) will end up not being the same. Note also that the right-hand side of $(5.1)$ can remain finite even when $\mathcal{L}^{2}(\operatorname{supp} \phi)=+\infty$.

2. By considering the positive and negative parts of $\phi$, it suffices to prove the lemma for $\phi \geq 0$. Fix such a function $\phi$, and for every $s \in \mathbb{R}$ define

$$
\chi_{s}(x)=\chi_{0 \leq s<\phi(x)}= \begin{cases}1 & \text { if } 0 \leq s<\phi(x) \\ 0 & \text { if not. }\end{cases}
$$

Next, for $s \in \mathbb{R}$ and $\delta>0$ define

$$
\chi_{s}^{\delta}(x)=\frac{1}{\delta} \int_{s}^{s+\delta} \chi_{\sigma}(x) \mathrm{d} \sigma
$$

Note that

$$
\begin{aligned}
\phi(x)=\int_{-\infty}^{\infty} \chi_{s}(x) \mathrm{d} s & =\frac{1}{\delta} \int_{0}^{\delta} \int_{-\infty}^{\infty} \chi_{s+\sigma}(x) \mathrm{d} s \mathrm{~d} \sigma \\
& =\int_{-\infty}^{\infty} \chi_{s}^{\delta}(x) \mathrm{d} s=\int_{-\delta}^{\|\phi\|_{\infty}} \chi_{s}^{\delta}(x) \mathrm{d} s
\end{aligned}
$$

In the last line I have used the fact that $\chi_{s}^{\delta} \equiv 0$ when $s<-\delta$ or $s>\|\chi\|_{\infty}$, which is an obvious consequence of the definition. I also claim that

$$
\chi_{s}^{\delta} \leq \chi_{\max \{s, 0\}}, \quad\left\|\nabla \chi_{\delta}\right\|_{\infty} \leq \frac{1}{\delta}\|\nabla \phi\|_{\infty} .
$$

The first of these follows from the fact that $\chi_{s+\sigma} \leq \chi_{\max \{s, 0\}}$ for every $s \in \mathbb{R}$ and $\sigma \geq 0$. To prove the second, fix $x, y \in U$. Then

$$
\begin{aligned}
\left|\chi_{s}^{\delta}(x)-\chi_{s}^{\delta}(y)\right| & =\frac{1}{\delta} \int_{s}^{s+\delta}\left|\chi_{\sigma}(x)-\chi_{\sigma}(y)\right| \mathrm{d} \sigma \\
& \leq \frac{1}{\delta} \int_{-\infty}^{\infty}\left|\chi_{\sigma}(x)-\chi_{\sigma}(y)\right| \mathrm{d} \sigma \\
& =\frac{1}{\delta}|\phi(x)-\phi(y)| \\
& \leq \frac{1}{\delta}\|\nabla \phi\|_{\infty}|x-y|
\end{aligned}
$$

establishing (5.4).

3. Now combine (5.3) and (5.2) and use (5.4) as follows:

$$
\begin{aligned}
\left|\int \phi J u \mathrm{~d} x\right| & =\left|\int_{-\delta}^{\|\phi\|_{\infty}}\left(\int \chi_{s}^{\delta} J u \mathrm{~d} x\right) \mathrm{d} s\right| \\
\leq & \int_{-\delta}^{\|\phi\|_{\infty}} \lambda \int_{\operatorname{supp} \chi_{s}^{\delta}} \frac{e_{\varepsilon}(u)}{|\ln \varepsilon|} \mathrm{d} x \\
& +C \varepsilon^{\alpha}\left(1+\frac{1}{\delta}\|\nabla \phi\|_{\infty}\right) \int_{-\delta}^{\|\phi\|_{\infty}}\left(1+\int_{\operatorname{supp} \chi_{s}^{\delta}} e_{\varepsilon}(u)\right)\left(1+\mathcal{L}^{2}\left(\operatorname{supp} \chi_{s}^{\delta}\right)\right) \mathrm{d} s .
\end{aligned}
$$

Using the first inequality of (5.4), the first integral on the right is bounded by

$$
\delta \lambda \int_{\operatorname{supp} \phi} \frac{e_{\varepsilon}(u)}{|\ln \varepsilon|} \mathrm{d} x+\lambda \int_{0}^{\|\phi\|_{\infty}} \int \chi_{s} \frac{e_{\varepsilon}(u)}{|\ln \varepsilon|} \mathrm{d} x \mathrm{~d} s=\lambda \int_{\operatorname{supp} \phi}(\phi+\delta) \frac{e_{\varepsilon}(u)}{|\ln \varepsilon|} \mathrm{d} x
$$


after using Fubini's Theorem as in (5.3). The second term is similarly estimated by

$$
C \varepsilon^{\alpha}\left(1+\frac{1}{\delta}\|\nabla \phi\|_{\infty}\right)\left(\|\phi\|_{\infty}+\delta+\int_{\operatorname{supp} \phi}(\phi+\delta) e_{\varepsilon}(u)\right)\left(1+\mathcal{L}^{2}(\operatorname{supp} \phi)\right)
$$

Now we take $\delta=\varepsilon^{\alpha / 2}$ and rename $\alpha$ to deduce (5.1), with a constant $\mathrm{C}$ depending on the support of $\phi$. To eliminate this dependence, let $\left\{\psi_{k}\right\}$ be a partition of unity on $\mathbb{R}^{2}$ such that each $\psi_{k}$ satisfies $\mathcal{L}^{2}\left(\operatorname{supp} \psi_{k}\right) \leq C$, $\left\|\nabla \psi_{k}\right\|_{\infty} \leq C, 0 \leq \psi_{k} \leq 1$. Then given an arbitrary $\phi$, clearly $\left|\int \phi J u\right| \leq \sum_{k}\left|\int\left(\psi_{k} \phi\right) J u\right|$. Taking $\delta=\varepsilon^{\alpha / 2}$ and renaming $\alpha$ as above, after summing the error terms (5.5) over $k$ one gets an error term of the desired form:

$$
C \varepsilon^{\alpha}\left(1+\|\phi\|_{W^{1, \infty}}\right)\left(\|\phi\|_{\infty}+1+\int_{\operatorname{supp} \phi}(|\phi|+1) e_{\varepsilon}(u)\right) .
$$

Lemma 9. There exist universal constants $C, \alpha>0$ such that, given any $U \subset \mathbb{R}^{3}$, and $u \in H^{1}\left(U ; \mathbb{R}^{2}\right)$,

$$
\left|\int_{U} \phi \wedge J u\right| \leq C \int|\phi| \frac{e_{\varepsilon}(u)}{|\ln \varepsilon|}+C \varepsilon^{\alpha}\left[\left(1+\|\nabla \phi\|_{\infty}\right)\left(\varepsilon^{\alpha}+\|\phi\|_{\infty}+\int_{\operatorname{supp} \phi}(|\phi|+1) e_{\varepsilon}(u)\right)\right]
$$

for all $\phi \in C_{c}^{0,1}\left(U ; \Lambda^{1} \mathbb{R}^{3}\right)$ and $\varepsilon \in(0,1]$.

Generalizations of this lemma hold in arbitrary dimensions, by essentially the same proof as given here.

It would be interesting to know whether one can take the constant $C$ in front of $\int|\phi| \frac{e_{\varepsilon}(u)}{\ln \varepsilon \mid}$ to be arbitrarily close to 1 , at the expense of making the constant in from of the error term larger. The proof here is not sharp enough to establish such an estimate; rather, it supplies a constant that can be arbitrarily close to 2 in 3 dimensions, with a worse constant in higher dimensions.

Proof. It suffices to prove that (5.6) holds for 1 -forms $\phi=\psi \mathrm{d} x^{i}$, for $i=1,2,3$. For $i=1$ for example, the estimate follows by writing

$$
\int\left(\psi \mathrm{d} x^{1}\right) \wedge J u=\int_{\mathbb{R}}\left(\int_{\left\{\left(x_{2}, x_{3}\right):\left(x_{1}, x_{2}, x_{3}\right) \in U\right\}} \psi u_{x_{2}} \wedge u_{x_{3}} \mathrm{~d} x_{2} \mathrm{~d} x_{3}\right) \mathrm{d} x_{1}
$$

and for each fixed $x_{1}$, applying the previous lemma (with some fixed value of $\lambda$, say $\lambda=2$ ) to the inner integral.

Remark 1. The above two lemmas as stated are not invariant under the scaling $\phi \mapsto \kappa \phi$, for $\kappa>0$. One can obtain a scale-invariant estimate by writing down the estimate obtained upon replacing $\phi$ by $\kappa \phi$, and then optimizing over $\kappa$.

The next lemma assembles known results due to a variety of authors.

Lemma 10. Suppose that $K$ is a compact subset of $\mathcal{D}$, and let $\left\{u_{\varepsilon}\right\}_{\varepsilon \in(0,1]} \subset H^{1}\left(\mathcal{D} ; \mathbb{R}^{2}\right)$ be a sequence of functions such that

$$
\frac{1}{|\ln \varepsilon|} \int_{K} e_{\varepsilon}\left(u_{\varepsilon}\right) \mathrm{d} x \leq C m_{\varepsilon}
$$

for some sequence of numbers $\left\{m_{\varepsilon}\right\}$ such that $1 \leq m_{\varepsilon} \leq C|\ln \varepsilon|$. Then $\left\{\frac{\star J u_{\varepsilon}}{m_{\varepsilon}}\right\}$ is precompact in the dual norm $C_{c}^{0, \alpha}(K)^{*}$ for every $\alpha>0$. Moreover, if $J$ is any limit in the above sense of a convergent subsequence (still denoted $\star J u_{\varepsilon} / m_{\varepsilon}$ ), then $J$ has finite mass in $K$. If in addition $\mu$ is a nonnegative measure such that $\frac{1}{m_{\varepsilon}|\ln \varepsilon|} e_{\varepsilon}\left(u_{\varepsilon}\right) \mathrm{d} x \rightarrow \mu$ weakly as measures, then

$$
\|J\| \ll \mu, \quad \text { and } \frac{\mathrm{d}\|J\|}{\mathrm{d} \mu} \leq 1 \quad \text { almost everywhere }
$$


where $\|J\|$ denotes the total variation measure associated with $J$. Also, if $m_{\varepsilon} \equiv 1$, and $\star J u_{\varepsilon}$ is a subsequence and $J$ a limiting current on $K$ such that $\star J u_{\varepsilon} \rightarrow J$ in the above sense, then $\frac{1}{\pi} J$ is 1-rectifiable and without boundary in $K$. Finally, for any $J$ such that $\frac{1}{\pi} J \in \stackrel{\circ}{\mathcal{R}}_{1}(\mathcal{D})$ and with finite mass in $\mathcal{D}$, there exists a sequence of functions $u_{\varepsilon} \in H^{1}\left(\mathcal{D} ; \mathbb{R}^{2}\right)$ such that $\star J u_{\varepsilon} \rightarrow J$ in $\cup_{\alpha>0} C_{c}^{0, \alpha}(K)^{*}$ and

$$
\frac{1}{\pi|\ln \varepsilon|} e_{\varepsilon}\left(u_{\varepsilon}\right) \mathrm{d} x \rightarrow\|J\| \quad \text { weakly as measures, as } \varepsilon \rightarrow 0 \text {. }
$$

It is known that (5.8) is sharp - in other words, that a statement like (5.9) holds - when $m_{\varepsilon} \ll|\ln \varepsilon|$ and not sharp when $m_{\varepsilon} \geq c|\ln \varepsilon| ;$ see [20].

Proof. Most of these results were proven first in [12] in the case $m_{\varepsilon} \equiv 1$ and, in the generality stated here (indeed in considerably greater generality) in [20]. The construction of a sequence satisfying (5.9) is given in $[4,5]$. The last two papers also include for example new proofs of the other results in the case $m_{\varepsilon}=1$, valid for more general energies than considered in [12]. For early results in similar spirit, see also [8].

\section{Asypmtotics of the Gross-Pitaevsky energy}

In this section I prove $\Gamma$-convergence results characterizing the asymptotic behavior of the functional $G_{\Omega_{\varepsilon}}^{\varepsilon}$.

Following [3], I will write $u_{\varepsilon}=\eta_{\varepsilon} v_{\varepsilon}$, where $\eta_{\varepsilon}$ is a nearly optimal vortex-free profile that will be defined in a moment. This leads to a nice splitting of the energy, see Lemma 11. The vortex-free profile $\eta_{\varepsilon}$ will have the form $\eta_{\varepsilon}=f_{\varepsilon} e^{i \Omega_{\varepsilon} S_{0}}$, for functions $f_{\varepsilon}, S_{0}$ that I now define. First, let $f_{\varepsilon}: \mathcal{D} \rightarrow \mathbb{R}$ minimize $G_{\Omega_{\varepsilon}}$ in $H_{0}^{1}(\mathcal{D} ; \mathbb{R})$. Note from the definition (3.2) that $j f=0$ for real-valued $f$, so that

$$
G_{\Omega_{\varepsilon}}^{\varepsilon}(f)=\int_{\mathcal{D}} \frac{1}{2}|\nabla f|^{2}+\frac{1}{4 \varepsilon^{2}}\left(\rho-f^{2}\right)^{2} \mathrm{~d} x \quad \text { for } f \in H_{0}^{1}(\mathcal{D} ; \mathbb{R}) .
$$

Since $G_{\Omega_{\varepsilon}}^{\varepsilon}(f)=G_{\Omega_{\varepsilon}}^{\varepsilon}(|f|)$ for real-valued $f$, we may assume that $f_{\varepsilon} \geq 0$ in $\mathcal{D}$. Using the Euler-Lagrange equation

$$
-\Delta f_{\varepsilon}+\frac{1}{\varepsilon^{2}}\left(f_{\varepsilon}^{2}-\rho\right) f_{\varepsilon}=0
$$

and the strong maximum principle one can deduce that in fact $f_{\varepsilon}$ is positive in $\mathcal{D}$. Next, define

$$
S_{0}(x):=C_{\mathcal{D}} x_{1} x_{2}, \quad C_{\mathcal{D}}:=\frac{a_{2}^{2}-a_{1}^{2}}{a_{2}^{2}+a_{1}^{2}}
$$

where $a_{1}, a_{2}$ are the parameters occurring in the definitions of $\rho$ and $\mathcal{D}$. As noted in [3], $S_{0} \operatorname{satisfies}^{4}$

$$
\nabla \cdot\left(\rho\left(\nabla S_{0}-V\right)\right)=0 \quad \text { in } \mathcal{D} .
$$

From (6.2) we see that $\rho\left(\nabla S_{0}-V\right)$ can be written in the form $\nabla \times \Xi$, and Aftalion and Rivière [3] observe that one can explicitly find $\Xi$. It is convenient to write the resulting equation in the form

$$
\rho\left(\nabla S_{0}-V\right)=-\frac{1}{2\left(1+\left(\frac{a_{1}}{a_{2}}\right)^{2}\right)} \star \mathrm{d}\left(\rho^{2} \mathrm{~d} x^{3}\right)
$$

where $\star$ is defined in $(3.1)$. Define

$$
\eta_{\varepsilon}:=f_{\varepsilon} e^{i \Omega_{\varepsilon} S_{0}}
$$

\footnotetext{
${ }^{4}$ The definition of $S_{0}$ is motivated by noting that $f_{\varepsilon}^{2} \approx \rho$ and that the Euler-Lagrange equation associated with the functional $S \mapsto G_{\Omega_{\varepsilon}}^{\varepsilon}\left(f_{\varepsilon} e^{i \Omega_{\varepsilon} S}\right)$ is $\nabla \cdot\left(f_{\varepsilon}^{2}(\nabla S-V)\right)=0$.
} 
Given $u \in H_{0}^{1}(\mathcal{D})$, I will often write $v_{\varepsilon}=u / \eta_{\varepsilon}$. Finally, I define

$$
H_{\varepsilon}\left(v_{\varepsilon}\right)=\int \frac{1}{2} f_{\varepsilon}^{2}\left|\nabla v_{\varepsilon}\right|^{2}+\frac{f_{\varepsilon}^{4}}{4 \varepsilon^{2}}\left(1-\left|v_{\varepsilon}\right|^{2}\right)^{2} .
$$

The main result of this section is the following. The statement is clearest in the case $m_{\varepsilon}=1$ (see (6.7)) which is also the case of most interest for us.

Theorem 2. Assume that

$$
G_{\Omega_{\varepsilon}}^{\varepsilon}\left(u_{\varepsilon}\right) \leq C|\ln \varepsilon|^{2}, \quad \text { and } \quad \frac{\Omega_{\varepsilon}}{\left(1+\left(a_{1} / a_{2}\right)^{2}\right)|\ln \varepsilon|} \rightarrow \Omega \quad \text { as } \varepsilon \rightarrow 0 .
$$

Assume also that $m_{\varepsilon}$ is a sequence of numbers such that

$$
\frac{1}{|\ln \varepsilon|} H_{\varepsilon}\left(v_{\varepsilon}\right) \leq C m_{\varepsilon}, \quad 1 \leq m_{\varepsilon} \leq C|\ln \varepsilon| .
$$

Then there exist $\varepsilon_{0}>0$ and $\alpha>0$ such that for every $\varepsilon \in\left(0, \varepsilon_{0}\right)$, there exists a current $\widetilde{J}_{\varepsilon} v_{\varepsilon}$ such that

$$
\partial\left(\widetilde{J}_{\varepsilon} v_{\varepsilon}\right)=0, \quad \mathbf{M}_{\rho}\left(\frac{\widetilde{J}_{\varepsilon} v_{\varepsilon}}{m_{\varepsilon}}\right) \leq C
$$

and $\widetilde{J}_{\varepsilon} v_{\varepsilon} \approx \star J v_{\varepsilon}$ in the sense that for $\|T\|_{X_{\varepsilon}}:=\sup \left\{T(\phi):\left\|\frac{\phi}{f_{\varepsilon}^{4}}\right\|_{\infty}+\left\|\frac{\nabla \phi}{f_{\varepsilon}^{2}}\right\|_{\infty} \leq 1\right\}$,

$$
\left\|\widetilde{J}_{\varepsilon} v_{\varepsilon}-\star J v_{\varepsilon}\right\|_{X_{\varepsilon}} \leq C \varepsilon^{\alpha} \quad \text { for some } \alpha>0 .
$$

(The constants $C$ in (6.9), (6.8) depend only on $C$ from (6.7).) In particular, by Lemma $2,\left\{\frac{\widetilde{J}_{\varepsilon} v_{\varepsilon}}{m_{\varepsilon}}\right\}$ is precompact in the $\mathbf{F}_{p, \rho, K}$ seminorm for every compact $K \subset \mathcal{D}$, and in addition $\left\{\frac{\star J v_{\varepsilon}}{m_{\varepsilon}}\right\}$ is precompact as a sequence of distributions. And if $J$ is any limit of a convergent subsequence $\frac{\star J v_{\varepsilon}}{m_{\varepsilon}}$, then

$$
\liminf \frac{1}{m_{\varepsilon}|\ln \varepsilon|}\left(G_{\Omega_{\varepsilon}}^{\varepsilon}\left(u_{\varepsilon}\right)-G_{\Omega_{\varepsilon}}^{\varepsilon}\left(\eta_{\varepsilon}\right)\right) \geq E_{\Omega}(J)
$$

In addition, if $m_{\varepsilon}=1$ for all $\varepsilon$, then $\frac{1}{\pi} J \in \dot{\mathcal{R}}_{1, \rho}(\mathcal{D})$. Moreover, for any $J$ such that $\frac{1}{\pi} J \in \stackrel{\circ}{\mathcal{R}}_{1, \rho}(\mathcal{D})$ there exists a sequence of functions $u_{\varepsilon}$ such that $\star J v_{\varepsilon} \rightarrow J$ in the above sense (where $v_{\varepsilon}=u_{\varepsilon} / \eta_{\varepsilon}$ ) and such that

$$
\lim \frac{1}{|\ln \varepsilon|}\left(G_{\Omega_{\varepsilon}}^{\varepsilon}\left(u_{\varepsilon}\right)-G_{\Omega_{\varepsilon}}^{\varepsilon}\left(\eta_{\varepsilon}\right)\right)=E_{\Omega}(J)
$$

The current $\widetilde{J}_{e} v_{\varepsilon}$ is obtained from $\frac{J v_{\varepsilon}}{m_{\varepsilon}}$ by modifying it in two ways. First, $J v_{\varepsilon}$ is regularized by convolution with a smoothing kernel $\omega_{\delta}$. This is necessary because $|\ln \varepsilon|^{-1} e_{\varepsilon}\left(v_{\varepsilon}\right)$ does not control $J v_{\varepsilon}$ in $L^{1}$, but does control $\left|\omega_{\delta} * J v_{\varepsilon}\right|$. Secondly, $\omega_{\delta} * J v_{\varepsilon}$ is modified near $\partial \mathcal{D}$. This is needed because (using the $L^{1}$-type control mentioned above)

$$
O(1)=\frac{H_{\varepsilon}\left(v_{\varepsilon}\right)}{|\ln \varepsilon| m_{\varepsilon}} \approx \frac{1}{|\ln \varepsilon| m_{\varepsilon}} \int_{\mathcal{D}} f_{\varepsilon}^{2} e_{\varepsilon}\left(v_{\varepsilon}\right) \mathrm{d} x \approx \int_{\mathcal{D}} f_{\varepsilon}^{2}\left|\omega_{\delta} * \frac{J v_{\varepsilon}}{m_{\varepsilon}}\right| \mathrm{d} x
$$

One can check that $f_{\varepsilon}^{2} / \rho \rightarrow 0$ at $\partial \mathcal{D}$, so the above estimate (even when carried out rigorously) cannot possibly provide uniform bounds over $\mathbf{M}_{\rho}\left(\omega_{\delta} * \frac{J v_{\varepsilon}}{m_{\varepsilon}}\right)=\int \rho\left|\omega_{\delta} * \frac{J v_{\varepsilon}}{m_{\varepsilon}}\right|$ due to the lack of control near $\partial \mathcal{D}$.

The starting point for the proof of the theorem is the following decomposition of the energy. A slightly different decomposition was the basis for the argument in [3]. Both this lemma and its counterpart in [3] rely on very useful ideas from [15]. 
Lemma 11. For any $u_{\varepsilon} \in H_{0}^{1}(\mathcal{D} ; \mathbb{C}), S_{0}$ and $v_{\varepsilon}$

$$
\begin{aligned}
G_{\Omega_{\varepsilon}}^{\varepsilon}\left(u_{\varepsilon}\right)-G_{\Omega_{\varepsilon}}^{\varepsilon}\left(\eta_{\varepsilon}\right)= & H_{\varepsilon}\left(v_{\varepsilon}\right)+\frac{\Omega_{\varepsilon}{ }^{2}}{2} \int f_{\varepsilon}^{2}\left(\left|v_{\varepsilon}\right|^{2}-1\right)\left(\left|\nabla S_{0}\right|^{2}-2 V \cdot \nabla S_{0}\right) \\
& +\Omega_{\varepsilon} \int\left\langle f_{\varepsilon}^{2}\left(\nabla S_{0}-V\right), j v_{\varepsilon}\right\rangle \\
:= & H_{\varepsilon}\left(v_{\varepsilon}\right)+I_{\varepsilon}\left(v_{\varepsilon}\right)+J_{\varepsilon}\left(v_{\varepsilon}\right) .
\end{aligned}
$$

The proof is essentially the same as that of the corresponding point in [3]. I present it here for the reader's convenience.

Proof. Note that $j u_{\varepsilon}=f_{\varepsilon}^{2} j v_{\varepsilon}+f_{\varepsilon}^{2}\left|v_{\varepsilon}\right|^{2} \Omega_{\varepsilon} \mathrm{d} S_{0}$, and

$$
\begin{aligned}
\left(\rho-\left|u_{\varepsilon}\right|^{2}\right)^{2} & =\left(\rho-f_{\varepsilon}^{2}+f_{\varepsilon}^{2}\left(1-\left|v_{\varepsilon}\right|^{2}\right)\right)^{2} \\
& =\left(\rho-f_{\varepsilon}^{2}\right)^{2}+f_{\varepsilon}^{4}\left(1-\left|v_{\varepsilon}\right|^{2}\right)^{2}+2\left(1-\left|v_{\varepsilon}\right|^{2}\right)\left(\rho-f_{\varepsilon}^{2}\right) f_{\varepsilon}^{2}
\end{aligned}
$$

and that

$$
\begin{aligned}
\left|\nabla u_{\varepsilon}\right|^{2}= & f_{\varepsilon}^{2}\left|\nabla v_{\varepsilon}\right|^{2}+\left|\nabla f_{\varepsilon}\right|^{2}+\left(\left|v_{\varepsilon}\right|^{2}-1\right)\left|\nabla f_{\varepsilon}\right|^{2} \\
& +\Omega_{\varepsilon}^{2} f_{\varepsilon}^{2}\left|v_{\varepsilon}\right|^{2}\left|\nabla S_{0}\right|^{2}+\frac{1}{2} \nabla\left(f_{\varepsilon}^{2}\right) \cdot \nabla|v|^{2}+2 \Omega_{\varepsilon} f_{\varepsilon}^{2}\left\langle\nabla S_{0}, j v_{\varepsilon}\right\rangle .
\end{aligned}
$$

Using these it is easy to check that

$$
\begin{aligned}
G_{\Omega_{\varepsilon}}^{\varepsilon}\left(u_{\varepsilon}\right)-G_{\Omega_{\varepsilon}}^{\varepsilon}\left(\eta_{\varepsilon}\right)= & H_{\varepsilon}\left(v_{\varepsilon}\right)+I_{\varepsilon}\left(v_{\varepsilon}\right)+J_{\varepsilon}\left(v_{\varepsilon}\right) \\
& +\frac{1}{2} \int\left[\left(\left|v_{\varepsilon}\right|^{2}-1\right)\left(\left|\nabla f_{\varepsilon}\right|^{2}-\frac{1}{\varepsilon^{2}}\left(\rho-f_{\varepsilon}^{2}\right) f_{\varepsilon}^{2}\right)+\frac{1}{2} \nabla\left(f_{\varepsilon}^{2}\right) \cdot \nabla\left|v_{\varepsilon}\right|^{2}\right] \mathrm{d} x
\end{aligned}
$$

So we only need to verify that the final term vanishes. To see this, use the identity $\left|\nabla f_{\varepsilon}\right|^{2}=\frac{1}{2} \Delta\left(f_{\varepsilon}^{2}\right)-f_{\varepsilon} \Delta f_{\varepsilon}$ and the Euler-Lagrange equation (6.1) of $f_{\varepsilon}$ to find that

$$
\left|\nabla f_{\varepsilon}\right|^{2}+\frac{1}{\varepsilon^{2}}\left(f_{\varepsilon}^{2}-\rho\right) f_{\varepsilon}^{2}=\frac{1}{2} \Delta\left(f_{\varepsilon}^{2}\right) .
$$

Now the lemma follows upon substituting and by integrating by parts; the boundary terms vanish because $f \in H_{0}^{1}$.

In order to prove that $f_{\varepsilon}$ converges uniformly to $\sqrt{\rho}$, I need a preliminary.

Lemma 12. $G_{\Omega_{\varepsilon}}^{\varepsilon}\left(f_{\varepsilon}\right) \leq C(1+|\ln \varepsilon|)$.

Proof. It suffices to exhibit any function $g_{\varepsilon} \in H_{0}^{1}(\mathcal{D})$ such that $G_{\Omega_{\varepsilon}}^{\varepsilon}\left(g_{\varepsilon}\right) \leq C(1+|\ln \varepsilon|)$. Look for $g_{\varepsilon}$ of the form $g_{\varepsilon}=\gamma_{\varepsilon}(\rho)$ for

where $\alpha>0$ will be selected below. Then

$$
\gamma_{\varepsilon}(s)= \begin{cases}\varepsilon^{-\alpha} s & \text { when } s \leq \varepsilon^{2 \alpha} \\ \sqrt{s} & \text { when } s \geq \varepsilon^{2 \alpha}\end{cases}
$$

$$
\int\left|\nabla g_{\varepsilon}\right|^{2}=\int \gamma_{\varepsilon}^{\prime}(\rho)^{2}|\nabla \rho|^{2} \leq C \int \gamma_{\varepsilon}^{\prime}(\rho)^{2}|\nabla \rho|=C \int_{0}^{\|\rho\|_{\infty}} \gamma_{\varepsilon}^{\prime}(s)^{2} \mathcal{H}^{2}\left(\rho^{-1}(s)\right) \mathrm{d} s
$$


by the coarea formula. Clearly the level sets $\rho^{-1}(s)$ have uniformly bounded $\mathcal{H}^{2}$ measure, so

$$
\int\left|\nabla g_{\varepsilon}\right|^{2} \leq C \int_{0}^{C} \gamma_{\varepsilon}^{\prime}(s)^{2} \mathrm{~d} s=1+\ln \left(C \varepsilon^{-2 \alpha}\right) .
$$

To estimate the other term, note that $|\nabla \rho| \geq C^{-1}>0$ in the region where $\rho-\gamma_{\varepsilon}^{2} \neq 0$, and so using the coarea formula as above,

$$
\int \frac{1}{4 \varepsilon^{2}}\left(\rho-\gamma_{\varepsilon}(\rho)^{2}\right)^{2} \leq C \int \frac{1}{4 \varepsilon^{2}}\left(\rho-\gamma_{\varepsilon}(\rho)^{2}\right)^{2}|\nabla \rho|=\frac{C}{\varepsilon^{2}} \int_{0}^{\varepsilon^{2 \alpha}}\left(s-\gamma_{\varepsilon}(s)^{2}\right)^{2}=C \varepsilon^{6 \alpha-2}
$$

after explicitly evaluating the integral. Taking $\alpha=1 / 3$ gives the desired estimate.

Lemma 13. There exists a constant $C>0$ such that

$$
\sqrt{\rho}(x)-C \varepsilon^{1 / 6} \leq f_{\varepsilon}(x)<\sqrt{\rho}(x)
$$

for all $x \in \mathcal{D}$ and $\varepsilon \in(0,1]$.

Proof. 1. Multiply the Euler-Lagrange equation (6.1) by $2 f_{\varepsilon}$ and rewrite to find that

$$
-\Delta\left(f_{\varepsilon}^{2}\right)+2\left|\nabla f_{\varepsilon}\right|^{2}+\frac{2}{\varepsilon^{2}}\left(f_{\varepsilon}^{2}-\rho\right) f_{\varepsilon}^{2}=0 .
$$

Define $v=f_{\varepsilon}^{2}-\rho$. From the explicit formula for $\rho$ we find that $\Delta \rho<0$, and so

$$
-\Delta v<-\Delta\left(f_{\varepsilon}^{2}\right) \leq-\frac{2}{\varepsilon^{2}} v(v+\rho)
$$

Also, $v=0$ on $\partial \mathcal{D}$, and so the strong maximum principle implies that $v<0$ in $\mathcal{D}$.

2. To prove the other inequality, note that since $0 \leq f_{\varepsilon}<\sqrt{\rho}$, the Euler-Lagrange equation (6.1) implies that $f_{\varepsilon}$ is superharmonic in $\mathcal{D}$. Now fix $x \in \mathcal{D}$, and write $\delta:=\sqrt{\rho(x)}-f_{\varepsilon}(x)$. I assert that if we define

$$
r=\min \left\{\frac{1}{2} \operatorname{dist}(x, \partial \mathcal{D}), C^{-1} \delta \operatorname{dist}(x, \partial \mathcal{D})^{1 / 2}\right\}
$$

for some suitable constant $C$, then $\sqrt{\rho}(y) \geq \sqrt{\rho}(x)-\delta / 2$ for $y \in B_{r}(x)$. Here is the proof: from the explicit form of $\rho$ one computes that

$$
|\nabla \sqrt{\rho}(x)|=\frac{1}{2}|\nabla \rho| \rho^{-1 / 2} \leq C \rho^{-1 / 2} \leq C \operatorname{dist}(x, \partial \mathcal{D})^{-1 / 2} .
$$

Since $r \leq \frac{1}{2} \operatorname{dist}(x, \partial \mathcal{D})$, clearly $|\nabla \sqrt{\rho}(y)| \leq C \operatorname{dist}(x, \partial \mathcal{D})^{-1 / 2}$ for all $y \in B_{r}(x)$, and so the mean value theorem and the definition of $r$ (with a suitable choice of the constant $C$ ) imply that $\sqrt{\rho}(y) \geq \sqrt{\rho}(x)-\delta / 2$ for all $y \in B_{r}$, which establishes the assertion.

Since $f_{\varepsilon}$ is superharmonic, it follows that

$$
\delta / 2=\sqrt{\rho}(x)-\delta / 2-f_{\varepsilon}(x) \leq \frac{1}{\left|B_{r}\right|} \int_{B_{r}(x)}\left(\sqrt{\rho}-f_{\varepsilon}\right) \mathrm{d} y .
$$

Thus using Jensen's inequality and the fact that $f_{\varepsilon} \geq 0$,

$$
\left(\frac{\delta}{2}\right)^{4} \leq \frac{1}{\left|B_{r}\right|} \int_{B_{r}(x)}\left(\sqrt{\rho}-f_{\varepsilon}\right)^{4} \mathrm{~d} y \leq \frac{1}{\left|B_{r}\right|} \int_{B_{r}(x)}\left(\rho-f_{\varepsilon}^{2}\right)^{2} \mathrm{~d} y \leq C \frac{\varepsilon^{2}}{r^{3}}|\ln \varepsilon| .
$$


The last inequality uses Lemma 12 . Clearly $\delta \leq \sqrt{\rho}(x) \leq C \operatorname{dist}(x, \partial \mathcal{D})^{1 / 2}$, and thus $\delta^{2} \leq C r$. Combining this with the above inequality yields

$$
\delta^{10} \leq C r^{3} \delta^{4} \leq C \varepsilon^{2}|\ln \varepsilon|
$$

which implies the desired inequality.

I now present the

Proof of Theorem 2. First, $\gamma$ denotes a constant that will be specified during the course of the proof. I start by requiring that $0<\gamma<1 / 6$. Throughout this proof I will write $\delta:=\varepsilon^{\gamma}$.

All constants $C$ in this proof are taken to be uniform for $0<\varepsilon<1 / 2$ unless mentioned otherwise; they may however depend on the constants in (6.7).

Step 1 (construction of $\widetilde{J}_{\varepsilon} v_{\varepsilon}$ ). In this step I modify $J v_{\varepsilon}$ as described following the statement of the theorem. In order to satisfy $\partial\left(\widetilde{J}_{\varepsilon} v_{\varepsilon}\right)=0$, the modification near $\partial \mathcal{D}$ mentioned there cannot be carried out by simple multiplication by a cutoff function; instead a more geometrically natural construction is used.

For $\sigma>0$, let $\mathcal{D}_{\sigma}:=\left\{x \in \mathcal{D}: \rho(x)>\|\nabla \rho\|_{\infty} \sigma\right\}$. Note that if $x \in \mathcal{D}_{\sigma}$ and $y \in B_{\tau}(x)$ for $\tau<\sigma$, then $y \in \mathcal{D}_{\sigma-\tau}$. Since $\gamma<1 / 6$, Lemma 13 implies that

$$
\left|1-\frac{\rho}{f_{\varepsilon}^{2}}\right| \leq C \varepsilon^{1 / 6-\gamma} \rightarrow 0 \text { in } \mathcal{D}_{\delta}
$$

Let $\zeta$ be a diffeomorphism from $\mathcal{D}_{2 \delta}$ onto $\mathcal{D}$ with the following properties:

$$
\begin{gathered}
\zeta(x)=x \quad \text { for } x \in \mathcal{D}_{4 \delta}, \\
\|\nabla \zeta\|_{\infty} \leq C, \quad \rho(x)\left|\nabla^{2} \zeta(x)\right| \leq C \text { for all } x \in \mathcal{D}_{2 \delta}
\end{gathered}
$$

and

$$
\rho(x) \geq c \sup _{y \in B_{\delta}(x)} \rho(\zeta(y)) \quad \text { for all } x \in \mathcal{D}_{\delta}
$$

(All the constants above are independent of $\varepsilon$.) Since $\rho(a x)=1-a^{2}+a^{2} \rho(x)$ for all $a>0$, such a diffeomorphism can be constructed by defining $\zeta(x)=x q(\rho(x))$, where $q$ satisfies

$$
q(s)= \begin{cases}1 & \text { if } s \geq 4 \delta\|\nabla \rho\|_{\infty}, \\ \left(1-2 \delta\|\nabla \rho\|_{\infty}\right)^{-1 / 2} & \text { when } s=2 \delta\|\nabla \rho\|_{\infty}\end{cases}
$$

and

$$
0 \geq q^{\prime}(s) \geq-C, \quad\left|q^{\prime \prime}(s)\right| \leq C / \delta .
$$

Such a function is easily seen to satisfy (6.14) and (6.15). To verify (6.16), note that if $x \in \mathcal{D}_{\delta}$ and $y \in B_{\delta}(x)$ then $\rho(\zeta(y)) \leq \rho(y) \leq \rho(x)+\|\nabla \rho\|_{\infty} \delta \leq 2 \rho(x)$.

Now let $\omega_{\delta}(x)=\delta^{-3} \omega(x / \delta)$ be a nonnegative symmetric smoothing kernel supported in $B_{\delta}(0)$, and for $\phi \in C_{c}^{\infty}\left(\mathcal{D} ; \Lambda^{1} \mathbb{R}^{3}\right)$ define

$$
\phi_{\varepsilon}^{1}=\omega_{\delta} * \zeta^{\#} \phi .
$$

For purposes of defining the convolution, set $\zeta^{\#} \phi=0$ in $\mathbb{R}^{3} \backslash \mathcal{D}$. Note that $\phi_{\varepsilon}^{1}$ is supported in $\mathcal{D}_{\delta}$. Finally, define $\widetilde{J}_{\varepsilon} v_{\varepsilon}:=\zeta_{\#} \omega_{\delta} *\left(\frac{\star J v_{\varepsilon}}{m_{\varepsilon}}\right)$, that is,

$$
\widetilde{J}_{\varepsilon} v_{\varepsilon}(\phi):=\star J v_{\varepsilon}\left(\omega_{\delta} * \zeta_{\#} \phi\right)=\star J v_{\varepsilon}\left(\phi_{\varepsilon}^{1}\right) \quad \forall \phi \in C_{c}^{\infty}\left(\mathcal{D} ; \Lambda^{1} \mathbb{R}^{3}\right)
$$

Step 2 (estimates of $\phi_{\varepsilon}^{1}$ ). I state these as 
Lemma 14. There exists a constant $C$ independent of $\varepsilon, \phi$, such that

$$
\left\|\frac{\phi_{\varepsilon}^{1}}{f_{\varepsilon}^{2}}\right\|_{\infty}+\varepsilon^{\gamma}\left\|\nabla \phi_{\varepsilon}^{1}\right\|_{\infty} \leq C\left\|\frac{\phi}{\rho}\right\|_{\infty}
$$

In addition, $\phi-\phi_{\varepsilon}^{1}$ can be decomposed as a sum of terms $\phi-\phi_{\varepsilon}^{1}=\phi_{\varepsilon}^{2}+\phi_{\varepsilon}^{3}$, such that

$$
\begin{gathered}
\varepsilon^{-\gamma}\left\|\frac{\phi_{\varepsilon}^{2}}{f_{\varepsilon}^{2}}\right\|_{\infty}+\left\|\nabla \phi_{\varepsilon}^{2}\right\|_{\infty} \leq C\left(\left\|\frac{\phi}{f_{\varepsilon}^{4}}\right\|_{\infty}+\left\|\frac{\nabla \phi}{f_{\varepsilon}^{2}}\right\|_{\infty}\right), \\
\left\|\frac{d \phi_{\varepsilon}^{3}}{f_{\varepsilon}^{2}}\right\|_{4} \leq C \varepsilon^{\gamma / 4}\left(\left\|\frac{\phi}{f_{\varepsilon}^{4}}\right\|_{\infty}+\left\|\frac{\nabla \phi}{f_{\varepsilon}^{2}}\right\|_{\infty}\right) .
\end{gathered}
$$

I give the proof at the end of this section. For now, I assume the lemma and continue with proof of Theorem 2. Step 3 (proof of (6.8)). By construction of the map $\phi \mapsto \phi_{\varepsilon}^{1}$ and the fact that $\partial\left(\star J v_{\varepsilon}\right)=0$ in $\mathcal{D}$,

$$
\partial \widetilde{J}_{\varepsilon} v_{\varepsilon}(\phi)=\widetilde{J}_{\varepsilon} v_{\varepsilon}(\partial \phi)=\star J v_{\varepsilon}\left((\partial \phi)_{\varepsilon}^{1}\right)=\star J v_{\varepsilon}\left(\partial \phi_{\varepsilon}^{1}\right)=\partial\left(\star J v_{\varepsilon}\right)\left(\phi_{\varepsilon}^{1}\right)=0
$$

for all compactly supported 1 -forms $\phi$. Next I claim that (once $\gamma$ is taken to be sufficiently small) if $\psi$ is any smooth 1-form with support in $\mathcal{D}_{\delta}$, then

$$
\left|\int \psi \wedge \frac{J v_{\varepsilon}}{m_{\varepsilon}}\right| \leq C\left\|\frac{\psi}{f_{\varepsilon}^{2}}\right\|_{\infty}+C \varepsilon^{\gamma}\left(1+\|\nabla \psi\|_{\infty}\right)\left(1+\|\psi\|_{\infty}\right) .
$$

For future reference I record that the constants $C$ above have the form

$$
C \frac{H_{\varepsilon}\left(v_{\varepsilon}\right)}{m_{\varepsilon}|\ln \varepsilon|}=C^{\prime} \frac{1}{m_{\varepsilon}|\ln \varepsilon|} \int_{\mathcal{D}_{\delta}} f_{\varepsilon}^{2} \frac{\left|\nabla v_{\varepsilon}\right|^{2}}{2}+\frac{f_{\varepsilon}^{4}}{4 \varepsilon^{2}}\left(\left|v_{\varepsilon}\right|^{2}-1\right)^{2} \mathrm{~d} x,
$$

where $C^{\prime}$ is an absolute constant. This implies (6.8), since for any smooth, compactly supported 1-form with $\|\phi / \rho\|_{\infty} \leq 1,(6.21)$ and (6.18) imply that

$$
\frac{\widetilde{J}_{\varepsilon} v_{\varepsilon}}{m_{\varepsilon}}(\phi)=\int \phi_{\varepsilon}^{1} \wedge \frac{J v_{\varepsilon}}{m_{\varepsilon}} \leq C\left\|\frac{\phi_{\varepsilon}^{1}}{f_{\varepsilon}^{2}}\right\|_{\infty}+C \varepsilon^{\gamma}\left(1+\left\|\nabla \phi_{\varepsilon}^{1}\right\|_{\infty}\right)\left(1+\left\|\phi_{\varepsilon}^{1}\right\|_{\infty}\right) \leq C,
$$

and by (3.6) this implies a uniform bound on $\mathbf{M}_{\rho}\left(\frac{\widetilde{J}_{\varepsilon} v_{\varepsilon}}{m_{\varepsilon}}\right)$.

To prove $(6.21)$, first define $\bar{\varepsilon}:=c \varepsilon^{1-(\gamma / 2)}$, where $c$ is chosen so that $\bar{\varepsilon}^{-2} \leq f_{\varepsilon}^{2} / \varepsilon^{2}$ on $\mathcal{D}_{\delta}$; this is possible due to $(6.13)$. The choice of $\bar{\varepsilon}$ implies that

$$
\int_{\mathcal{D}_{\delta}} f_{\varepsilon}^{2} e_{\bar{\varepsilon}}\left(v_{\varepsilon}\right) \leq \int f_{\varepsilon}^{2} \frac{\left|\nabla v_{\varepsilon}\right|^{2}}{2}+\frac{f_{\varepsilon}^{4}}{4 \varepsilon^{2}}\left(\left|v_{\varepsilon}\right|^{2}-1\right)^{2}=H_{\varepsilon}\left(v_{\varepsilon}\right) .
$$

For $\psi$ with support in $\mathcal{D}_{\delta}$, use (5.6) $u$ replaced by $v_{\varepsilon}$ and with $\varepsilon$ replaced by $\bar{\varepsilon}$ to find that $\int \psi \wedge \frac{J v_{\varepsilon}}{m_{\varepsilon}} \leq A+B$, with

$$
\begin{aligned}
& A=C \int_{\mathcal{D}_{\delta}}|\psi| \frac{e_{\bar{\varepsilon}}\left(v_{\varepsilon}\right)}{m_{\varepsilon}|\ln \bar{\varepsilon}|}, \\
& B=\frac{C}{m_{\varepsilon}} \bar{\varepsilon}^{\alpha}\left(1+\|\nabla \psi\|_{\infty}\right)\left(\bar{\varepsilon}^{\alpha}+\|\psi\|_{\infty}+\int_{\mathcal{D}_{\delta}}(|\psi|+1) e_{\bar{\varepsilon}}\left(v_{\varepsilon}\right)\right) .
\end{aligned}
$$


The constant $\alpha$ comes from Lemma 9. In view of (6.23) and (6.7),

$$
|A| \leq\left\|\frac{\psi}{f_{\varepsilon}^{2}}\right\|_{\infty} \int_{\mathcal{D}_{\delta}} f_{\varepsilon}^{2} \frac{e_{\bar{\varepsilon}}\left(v_{\varepsilon}\right)}{m_{\varepsilon}|\ln \bar{\varepsilon}|} \leq\left\|\frac{\psi}{f_{\varepsilon}^{2}}\right\|_{\infty} \frac{H_{\varepsilon}\left(v_{\varepsilon}\right)}{m_{\varepsilon}|\ln \bar{\varepsilon}|} \leq C\left\|\frac{\psi}{f_{\varepsilon}^{2}}\right\|_{\infty}
$$

As for the other term, (6.13) and the argument of (6.24) imply that

$$
\int_{\mathcal{D}_{\delta}}(|\psi|+1) e_{\bar{\varepsilon}}\left(v_{\varepsilon}\right) \leq C m_{\varepsilon}|\ln \bar{\varepsilon}|\left\|\frac{|\psi|+1}{f_{\varepsilon}^{2}}\right\|_{\infty} \leq C \varepsilon^{-\gamma} m_{\varepsilon}|\ln \varepsilon|\left(\|\psi\|_{\infty}+1\right) .
$$

As a result, recalling the definition of $\bar{\varepsilon}$,

$$
B \leq C \varepsilon^{\alpha(1-(\gamma / 2))}\left(1+\|\nabla \psi\|_{\infty}\right) \varepsilon^{-\gamma}|\ln \varepsilon|\left(1+\|\psi\|_{\infty}\right)
$$

So if $\gamma<\alpha / 4$. then $B \leq C \varepsilon^{\gamma}\left(1+\|\nabla \psi\|_{\infty}\right)\left(1+\|\psi\|_{\infty}\right)$. With (6.24), this proves (6.21).

Step 4 (proof of (6.9)).

First, using Lemma 14,

$$
\frac{1}{m_{\varepsilon}}\left(\star J v_{\varepsilon}-\widetilde{J}_{\varepsilon} v_{\varepsilon}\right)(\phi)=\int\left(\phi-\phi_{\varepsilon}^{1}\right) \wedge \frac{J v_{\varepsilon}}{m_{\varepsilon}}=\int \phi_{\varepsilon}^{2} \wedge \frac{J v_{\varepsilon}}{m_{\varepsilon}}+\int \phi_{\varepsilon}^{3} \wedge \frac{J v_{\varepsilon}}{m_{\varepsilon}}=: I_{2}+I_{3} .
$$

From (6.21) and (6.19), it follows that

$$
\left|I_{2}\right| \leq C \varepsilon^{\gamma}\left(\left\|\frac{\phi}{f_{\varepsilon}^{4}}\right\|_{\infty}+\left\|\frac{\nabla \phi}{f_{\varepsilon}^{2}}\right\|_{\infty}\right)
$$

To control $I_{3}$, I need the estimate

$$
\left\|f_{\varepsilon}^{2} j v_{\varepsilon}\right\|_{4 / 3} \leq C(|\ln \varepsilon|+1) .
$$

This is proved by first noting that $f_{\varepsilon}^{2} j v_{\varepsilon}=j u_{\varepsilon}+\Omega_{\varepsilon}\left|u_{\varepsilon}\right|^{2} \mathrm{~d} S_{0}$ - this is a short calculation - and using Hölder's inequality $\left\|j u_{\varepsilon}\right\|_{4 / 3} \leq\left\|u_{\varepsilon}\right\|_{4}\left\|\nabla u_{\varepsilon}\right\|_{2}$ and bounds that follow easily from (6.6) and Lemma 1. For future reference I record the fact that the constant $C$ in (6.26) depends only on the assumed bounds in (6.6), and not on the assumption $H_{\varepsilon}\left(v_{\varepsilon}\right) \leq C|\ln \varepsilon|$.

Now recalling that $\star J v_{\varepsilon}=\frac{1}{2} \partial\left(\star j v_{\varepsilon}\right)$ or, equivalently, using the identity $J v_{\varepsilon}=\frac{1}{2} d j v_{\varepsilon}$ and integrating by parts, we obtain (since $m_{\varepsilon} \geq 1$ )

$$
\left|I_{3}\right|=\frac{1}{2}\left|\int \mathrm{d} \phi_{\varepsilon}^{3} \wedge j v_{\varepsilon}\right| \leq\left\|\frac{\mathrm{d} \phi_{\varepsilon}^{3}}{f_{\varepsilon}^{2}}\right\|_{4}\left\|f_{\varepsilon}^{2} j v_{\varepsilon}\right\|_{4 / 3} \leq C \varepsilon^{\gamma / 6}\left(\left\|\frac{\phi}{f_{\varepsilon}^{4}}\right\|_{\infty}+\left\|\frac{\nabla \phi}{f_{\varepsilon}^{2}}\right\|_{\infty}\right) .
$$

by (6.20) and (6.26). With (6.25) this proves (6.9).

Step 5 (properties of limiting currents). In this step I establish properties of any limit $J$ of a convergent subsequence, still denoted $\frac{1}{m_{\varepsilon}} \star J v_{\varepsilon}$.

First, it is clear from $(6.9),(6.8)$, and the compactness Lemma 2 that if $\frac{1}{m_{\varepsilon}} \star J v_{\varepsilon} \rightarrow J$ in the sense of distributions, then

$$
\mathbf{F}_{p, \rho, K}\left(\frac{\widetilde{J}_{\varepsilon} v_{\varepsilon}}{m_{\varepsilon}}-J\right) \rightarrow 0
$$

for all compact $K \subset \mathcal{D}$, and in addition $\frac{1}{m_{\varepsilon}} \widetilde{J}_{\varepsilon} v_{\varepsilon} \rightarrow J$ in the sense of distributions. Since $\mathbf{M}_{\rho}\left(\frac{1}{m_{\varepsilon}} \widetilde{J}_{\varepsilon} v_{\varepsilon}\right) \leq C$ and $\partial \widetilde{J}_{\varepsilon} v_{\varepsilon}=0$, these properties are inherited by $J$, and so $J \in \dot{\mathcal{M}}_{1, \rho}(\mathcal{D})$.

The final property to be proved is that if $m_{\varepsilon} \leq C$ and $J$ is a limit of $\star J v_{\varepsilon}$, then $J \in \stackrel{\circ}{\mathcal{R}}_{1, \rho}$. This however is a direct consequence of Lemma 10. 
Step 6 (proof of (6.10)). I continue to assume that $\frac{1}{m_{\varepsilon}} \star J v_{\varepsilon} \rightarrow J$ along a subsequence, in the sense of distributions, and hence that (6.27) holds. In view of the decomposition of the energy given in Lemma 11, it is natural to break the proof of the lower bound (6.10) into three parts:

Step 6a (lower bound for $H_{\varepsilon}\left(v_{\varepsilon}\right)$ ). I will prove that

$$
\liminf _{\varepsilon \rightarrow 0} \frac{1}{m_{\varepsilon}|\ln \varepsilon|} H_{\varepsilon}\left(v_{\varepsilon}\right) \geq \int_{\mathcal{D}} \rho \mathrm{d}\|J\| .
$$

Note that the right-hand side is finite as a result of Step 5.

Fix any $W \subset \subset \mathcal{D}$, and define $d=\min \left\{f_{\varepsilon}(x): 0<\varepsilon \leq 1, x \in \bar{W}\right\}$. Lemma 13 implies that $d>0$. Given $\varepsilon>0$, define $\tilde{\varepsilon}=\varepsilon / d$. Then for $x \in W$,

$$
\frac{1}{m_{\varepsilon}|\ln \varepsilon|} f_{\varepsilon}^{2}\left(\frac{\left|\nabla v_{\varepsilon}\right|^{2}}{2}+\frac{f_{\varepsilon}^{2}}{4 \varepsilon^{2}}\left(\left|v_{\varepsilon}\right|^{2}-1\right)^{2}\right) \geq \rho_{\tilde{\varepsilon}} \frac{e_{\tilde{\varepsilon}}\left(v_{\varepsilon}\right)}{m_{\varepsilon}|\ln \tilde{\varepsilon}|}, \quad \rho_{\tilde{\varepsilon}}:=f_{\varepsilon}^{2} \frac{|\ln \tilde{\varepsilon}|}{|\ln \varepsilon|} .
$$

Writing $\tilde{v}_{\tilde{\varepsilon}}:=v_{\varepsilon}$ and similarly $\tilde{m}_{\tilde{\varepsilon}}=m_{\varepsilon}$, this yields

$$
C \geq \frac{1}{m_{\varepsilon}|\ln \varepsilon|} H_{\varepsilon}\left(v_{\varepsilon}\right) \geq \int_{W} \rho_{\tilde{\varepsilon}} \frac{e_{\tilde{\varepsilon}}\left(\tilde{v}_{\tilde{e}}\right)}{\tilde{m}_{\tilde{\varepsilon}}|\ln \tilde{\varepsilon}|} .
$$

Since $\rho_{\tilde{\varepsilon}}$ is bounded away from zero on $W$ for $\varepsilon$ sufficiently small, this implies that that $\frac{1}{\tilde{m}_{\tilde{\varepsilon}} \ln \tilde{\varepsilon}} e_{\tilde{\varepsilon}}\left(\tilde{v}_{\tilde{\varepsilon}}\right)$ is uniformly bounded as a sequence on measures on $W$. Upon passing to a subsequence we may thus assume that $\frac{1}{\tilde{m}_{\tilde{\varepsilon}}|\ln \tilde{\varepsilon}|} e_{\tilde{\varepsilon}}\left(\tilde{v}_{\tilde{\varepsilon}}\right)$ converges weakly as measures to a limit $\mu$. Then (5.8) implies that $\|J\| \ll \mu$, with $d\|J\| / \mathrm{d} \mu \leq 1$ almost everywhere. Rewriting the above inequality yields

$$
\frac{1}{m_{\varepsilon}|\ln \varepsilon|} H_{\varepsilon}\left(v_{\varepsilon}\right) \geq \int_{W} \rho \frac{e_{\tilde{\varepsilon}}\left(\tilde{v}_{\tilde{\varepsilon}}\right)}{\tilde{m}_{\tilde{\varepsilon}}|\ln \tilde{\varepsilon}|}+\int_{W}\left(\rho_{\varepsilon}-\rho\right) \frac{e_{\tilde{\varepsilon}}\left(\tilde{v}_{\tilde{\varepsilon}}\right)}{\tilde{m}_{\tilde{\varepsilon}}|\ln \tilde{\varepsilon}|} .
$$

Since $\rho_{\varepsilon} \rightarrow \rho$ uniformly, it follows that

$$
\liminf _{\varepsilon} \frac{1}{m_{\varepsilon}|\ln \varepsilon|} H_{\varepsilon}\left(v_{\varepsilon}\right) \geq \int_{W} \rho \mathrm{d} \mu \geq \int_{W} \rho \mathrm{d}\|J\| .
$$

Since this holds for all $W \subset \subset \mathcal{D}$, this implies that (6.28) holds.

Step $6 \mathbf{b}$ (lower bound for $I_{\varepsilon}\left(v_{\varepsilon}\right)$ ). Since $S_{0}$ and $V$ are fixed, smooth functions and $f_{\varepsilon}^{2} \leq \rho$ for all $\varepsilon$,

$$
\begin{aligned}
I_{\varepsilon}\left(v_{\varepsilon}\right) & =\frac{\Omega_{\varepsilon}{ }^{2}}{2} \int f_{\varepsilon}^{2}\left(\left|v_{\varepsilon}\right|^{2}-1\right)\left(\left|\nabla S_{0}\right|^{2}-2 V \cdot \nabla S_{0}\right) \\
& \leq C \Omega_{\varepsilon}{ }^{2}\left\|f_{\varepsilon}^{2}\left(\left|v_{\varepsilon}\right|^{2}-1\right)\right\|_{2} \\
& \leq C \varepsilon \Omega_{\varepsilon}^{2}\left(H_{\varepsilon}\left(v_{\varepsilon}\right)\right)^{1 / 2} .
\end{aligned}
$$

And the right-hand side tends to zero as $\varepsilon \rightarrow 0$ as a result of the assumed bounds (6.6), (6.7) on $H_{\varepsilon}\left(v_{\varepsilon}\right)$ and $\Omega_{\varepsilon}$.

Step 6c (limit of $\left.J_{\varepsilon}\left(v_{\varepsilon}\right)\right)$. I will finish Step 6 by proving that

$$
\frac{1}{m_{\varepsilon}|\ln \varepsilon|} J_{\varepsilon}\left(v_{\varepsilon}\right)=\frac{1}{m_{\varepsilon}|\ln \varepsilon|} \Omega_{\varepsilon} \int\left\langle f_{\varepsilon}^{2}\left(\nabla S_{0}-V\right), j v_{\varepsilon}\right\rangle \longrightarrow-\Omega J\left(\rho^{2} \mathrm{~d} x^{3}\right) .
$$

By assumption $\Omega_{\varepsilon} /|\ln \varepsilon| \rightarrow\left(1+\left(a_{1} / a_{2}\right)^{2}\right) \Omega$, so I only need to prove that

$$
\int\left\langle f_{\varepsilon}^{2}\left(\nabla S_{0}-V\right), \frac{j v_{\varepsilon}}{m_{\varepsilon}}\right\rangle \longrightarrow \frac{-1}{1+\left(a_{1} / a_{2}\right)^{2}} J\left(\rho^{2} \mathrm{~d} x^{3}\right) .
$$


Let $\chi_{\varepsilon} \in C_{0}^{\infty}(\mathcal{D})$ be a function such that $0 \leq \chi \leq 1$,

$$
\chi_{\varepsilon}(x)=\left\{\begin{array}{ll}
1 & \text { if } x \in \mathcal{D}_{2 \delta} \\
0 & \text { if } x \notin \mathcal{D}_{\delta}
\end{array} \quad|\nabla \chi| \leq C / \delta\right.
$$

where I continue to write $\delta=\varepsilon^{\gamma}$. The definition of $\chi$ also implies that $\rho\left|\nabla \chi_{\varepsilon}\right| \leq C$.

I decompose the left-hand side of (6.31) as

$$
\begin{aligned}
\int\left\langle f_{\varepsilon}^{2}\left(\nabla S_{0}-V\right), \frac{j v_{\varepsilon}}{m_{\varepsilon}}\right\rangle= & \int \chi_{\varepsilon}\left\langle\rho\left(\nabla S_{0}-V\right), \frac{j v_{\varepsilon}}{m_{\varepsilon}}\right\rangle \\
& +\int \chi_{\varepsilon}\left\langle\left(f_{\varepsilon}^{2}-\rho\right)\left(\nabla S_{0}-V\right), \frac{j v_{\varepsilon}}{m_{\varepsilon}}\right\rangle \\
& +\int\left(1-\chi_{\varepsilon}\right)\left\langle f_{\varepsilon}^{2}\left(\nabla S_{0}-V\right), \frac{j v_{\varepsilon}}{m_{\varepsilon}}\right\rangle
\end{aligned}
$$

The last two terms are error terms and are easily estimated. For example, (6.13) readily implies that $\| \chi_{\varepsilon}(1-$ $\left.\frac{\rho}{f_{\varepsilon}^{2}}\right) \|_{4} \leq C \varepsilon^{(1 / 6)-\gamma}$, and so by Hölder's inequality and (6.26),

$$
\int \chi_{\varepsilon}\left\langle\left(f_{\varepsilon}^{2}-\rho\right)\left(\nabla S_{0}-V\right), \frac{j v_{\varepsilon}}{m_{\varepsilon}}\right\rangle \leq C \int \chi_{\varepsilon}\left|1-\frac{\rho}{f_{\varepsilon}^{2}}\right| \frac{f_{\varepsilon}^{2}\left|j v_{\varepsilon}\right|}{m_{\varepsilon}} \leq C \varepsilon^{(1 / 6)-\gamma}|\ln \varepsilon| .
$$

And similarly one can use (6.26) and the support properties of $\chi_{\varepsilon}$ to find that

$$
\int\left(1-\chi_{\varepsilon}\right)\left\langle f_{\varepsilon}^{2}\left(\nabla S_{0}-V\right), \frac{j v_{\varepsilon}}{m_{\varepsilon}}\right\rangle \leq \frac{C}{m_{\varepsilon}}\left\|1-\chi_{\varepsilon}\right\|_{4}\left\|f_{\varepsilon}^{2} j v_{\varepsilon}\right\|_{4 / 3} \leq \frac{C}{m_{\varepsilon}} \varepsilon^{\gamma / 4}|\ln \varepsilon| .
$$

To estimate the first term in (6.33), use (6.3) to write

$$
\begin{aligned}
\int \chi_{\varepsilon}\left\langle\rho\left(d S_{0}-V\right), \frac{j v_{\varepsilon}}{m_{\varepsilon}}\right\rangle & =\frac{-1}{2+2\left(a_{1} / a_{2}\right)^{2}} \int \chi_{\varepsilon}\left\langle\star \mathrm{d}\left(\rho^{2} \mathrm{~d} x^{3}\right), \frac{j v_{\varepsilon}}{m_{\varepsilon}}\right\rangle \\
& =\frac{-1}{2+2\left(a_{1} / a_{2}\right)^{2}}\left(\int\left\langle\star \mathrm{d}\left(\chi_{\varepsilon} \rho^{2} \mathrm{~d} x^{3}\right), \frac{j v_{\varepsilon}}{m_{\varepsilon}}\right\rangle-\int\left\langle\star\left(\rho^{2} \mathrm{~d} \chi_{\varepsilon} \wedge \mathrm{d} x^{3}\right), \frac{j v_{\varepsilon}}{m_{\varepsilon}}\right\rangle\right) .
\end{aligned}
$$

The second term is an error term and is handled like the error terms above. This leaves only $\frac{-1}{2+2\left(a_{1} / a_{2}\right)^{2}} \int\left\langle\star \mathrm{d}\left(\chi_{\varepsilon} \rho^{2} \mathrm{~d} x^{3}\right), \frac{j v_{\varepsilon}}{m_{\varepsilon}}\right\rangle$. On account of (3.4) and the fact that $\star J u=\frac{1}{2} \partial(\star j u)$, our estimate so far can be expressed as

$$
\int\left\langle f_{\varepsilon}^{2}\left(\nabla S_{0}-V\right), \frac{j v_{\varepsilon}}{m_{\varepsilon}}\right\rangle=\frac{-1}{1+\left(a_{1} / a_{2}\right)^{2}} \frac{\star J v_{\varepsilon}}{m_{\varepsilon}}\left(\chi_{\varepsilon} \rho^{2} \mathrm{~d} x^{3}\right)+O\left(\varepsilon^{\alpha}\right)
$$

for some $\alpha>0$. To estimate the right-hand side, note that (6.13) implies that there exists a constant $C$, as always independent of $\varepsilon$, such that

Then (6.9) implies that

$$
\left\|\frac{\chi_{\varepsilon} \rho^{2}}{f_{\varepsilon}^{4}}\right\|_{\infty}+\left\|\frac{\nabla\left(\chi_{\varepsilon} \rho^{2}\right)}{f_{\varepsilon}^{2}}\right\|_{\infty} \leq C
$$

$$
\frac{1}{m_{\varepsilon}}\left(\star J v_{\varepsilon}-\widetilde{J}_{\varepsilon} v_{\varepsilon}\right)\left(\chi_{\varepsilon} \rho^{2} \mathrm{~d} x^{3}\right) \leq C \varepsilon^{\alpha}
$$

for some $C, \alpha>0$. And using properties of the support of $1-\chi_{\varepsilon}$,

$$
\frac{1}{m_{\varepsilon}}\left|\widetilde{J}_{\varepsilon} v_{\varepsilon}\left(\left(1-\chi_{\varepsilon}\right) \rho^{2} \mathrm{~d} x^{3}\right)\right| \leq \frac{1}{m_{\varepsilon}} \int_{\left\{\rho<2 \delta\|\nabla \rho\|_{\infty}\right\}} \rho^{2} \mathrm{~d}\left\|\widetilde{J}_{\varepsilon} v_{\varepsilon}\right\| \leq C \delta \mathbf{M}_{\rho}\left(\frac{\widetilde{J}_{\varepsilon} v_{\varepsilon}}{m_{\varepsilon}}\right) \leq C \delta
$$


in view of (6.8). Finally, the fact (6.27) that $\frac{1}{m_{\varepsilon}} \widetilde{J}_{\varepsilon}\left(v_{\varepsilon}\right) \rightarrow J$ in $\mathbf{F}_{p, \rho, K}$ and Lemma 5 combine to show that $\widetilde{J}_{\varepsilon} v_{\varepsilon}\left(\rho^{2} \mathrm{~d} x^{3}\right) \rightarrow J\left(\rho^{2} \mathrm{~d} x^{3}\right)$ as $\varepsilon \rightarrow 0$.

The previous inequality and (6.35) therefore show that $\frac{\star J v_{\varepsilon}}{m_{\varepsilon}}\left(\chi_{\varepsilon} \rho^{2} \mathrm{~d} x^{3}\right) \rightarrow J\left(\rho^{2} \mathrm{~d} x^{3}\right)$, and with (6.34) this proves (6.31) and completes Step 6c.

Now (6.10) follows immediately from combining Steps 6a through $6 \mathrm{c}$ and using the decomposition of $G_{\Omega_{\varepsilon}}^{\varepsilon}$ from Lemma 11.

For future use I remark that the estimates given in this step combine to show that

$$
\left|J_{\varepsilon}\left(v_{\varepsilon}\right)+\frac{\Omega_{\varepsilon}}{1+\left(a_{1} / a_{2}\right)^{2}} \widetilde{J}_{\varepsilon} v_{\varepsilon}\left(\rho^{2} \mathrm{~d} x^{3}\right)\right| \leq C \varepsilon^{\alpha}
$$

for some $\alpha>0$, and an inspection of the proof shows that $\alpha$ is independent of $u_{\varepsilon}$, while $C$ depends only on the bounds in (6.6).

Step 7 (proof of (6.11)). To complete the proof I need to establish the existence of sequences for which the lower bound is attained in the case when the limiting measure $J$ is rectifiable and $H_{\varepsilon}\left(v_{\varepsilon}\right) \leq C|\ln \varepsilon|$.

Step 7a (the case of finite mass). First consider $J$ such that $\frac{1}{\pi} J \in \mathcal{R}_{1, \rho}$ and $J$ has finite mass in $\mathcal{D}$. For such $J$, let $v_{\varepsilon}$ be a sequence such that $\star J v_{\varepsilon} \rightarrow J$ in the sense of Lemma 10 and such that (5.9) holds, and define $u_{\varepsilon}:=\eta_{\varepsilon} v_{\varepsilon}$. We have already seen that $\left\{\star J v_{\varepsilon}\right\}_{\varepsilon \in(0,1]}$ is precompact in the sense of distributions and (up to an error term that vanishes as in (6.9)) in the $\mathbf{F}_{p, \rho, K}$ seminorm for all $K \subset \subset \mathcal{D}$, and in view of the construction of $v_{\varepsilon}$, the only possible limit in any of these topologies is $J$. Thus $\star J v_{\varepsilon} \rightarrow J$ in the desired sense.

To establish (6.11), note that for $m_{\varepsilon} \equiv 1$ and $\star J v_{\varepsilon} \rightarrow J$ as above, since $\left\|f_{\varepsilon}^{2}\right\|_{\infty} \leq\|\rho\|_{\infty} \leq 1$.

$$
\frac{1}{|\ln \varepsilon|} H_{\varepsilon}\left(v_{\varepsilon}\right) \leq \int_{\mathcal{D}} \rho \frac{e_{\varepsilon}\left(v_{\varepsilon}\right)}{|\ln \varepsilon|} \rightarrow \int_{\mathcal{D}} \rho \mathrm{d}\|J\|
$$

by (5.9), and combining this with (6.29), (6.30) yields

$$
\begin{aligned}
\limsup \frac{1}{|\ln \varepsilon|}\left(G_{\Omega_{\varepsilon}}^{\varepsilon}\left(u_{\varepsilon}\right)-G_{\Omega_{\varepsilon}}^{\varepsilon}\left(\eta_{\varepsilon}\right)\right) & =\limsup \frac{1}{|\ln \varepsilon|}\left(H_{\varepsilon}\left(v_{\varepsilon}\right)+I_{\varepsilon}\left(v_{\varepsilon}\right)+J_{\varepsilon}\left(v_{\varepsilon}\right)\right) \\
& \leq E_{\Omega}(J) .
\end{aligned}
$$

The opposite inequality (6.10) has already been established in step 6 .

Step $7 \mathbf{b}$ (the general case).

By a standard diagonalization argument, the general case follows from the case of currents with finite mass once it is verified that

$$
\mathcal{F}:=\left\{T: \frac{1}{\pi} T \in \dot{\mathcal{R}}_{1, \rho}(\mathcal{D}), T \text { has finite mass }\right\}
$$

is dense in $\pi \dot{\mathcal{R}}_{1, \rho}(\mathcal{D}):=\left\{T: \frac{1}{\pi} T \in \dot{\mathcal{R}}_{1, \rho}(\mathcal{D})\right\}$ in the sense that for every $T \in \pi \stackrel{\circ}{\mathcal{R}}_{1, \rho}(\mathcal{D})$, there exists a sequence of currents $\left\{T_{\sigma}\right\}_{\sigma \in(0,1]} \subset \mathcal{F}$ such that

$$
\mathbf{F}_{p, \rho, K}\left(T_{\sigma}-T\right) \rightarrow 0, \text { for every compact } K \subset \mathcal{D}, \quad \text { and } E_{\Omega}\left(T_{\sigma}\right) \rightarrow E_{\Omega}(T)
$$

as $\sigma \rightarrow 0$. This is proved in Lemma 15 in Section 9 .

I conclude this section by establishing the lemma used in step 2 of the proof of Theorem 2 :

Proof of Lemma 14. Recall that $\phi_{\varepsilon}^{1}=\omega_{\delta} * \zeta^{\#} \phi$, where $\zeta$ satisfies (6.14), (6.15), and (6.16), and $\omega_{\delta}$ is a smoothing kernel. I continue to write $\delta=\varepsilon^{\gamma}$, and I define

$$
\phi_{\varepsilon}^{2}=\zeta^{\#} \phi-\omega_{\delta} * \zeta^{\#} \phi, \quad \phi_{\varepsilon}^{3}=\phi-\zeta^{\#} \phi,
$$

so that $\phi=\phi_{\varepsilon}^{1}+\phi_{\varepsilon}^{2}+\phi_{\varepsilon}^{3}$. 
Note that $\phi_{\varepsilon}^{1}$ and $\phi_{\varepsilon}^{2}$ are supported in $\mathcal{D}_{\delta}$, and that (6.13) and (6.16) imply that

$$
f_{\varepsilon}^{2}(x) \geq C^{-1} \rho(x) \geq C^{-1} \sup _{y \in B_{\delta}(x)} \rho(\zeta(y)) \quad \text { for } x \in \mathcal{D}_{\delta} .
$$

3. To estimate $\left\|\phi_{\varepsilon}^{1} / f_{\varepsilon}^{2}\right\|_{\infty}$, note that

$$
\left|\phi_{\varepsilon}^{1}(x)\right| \leq \omega_{\delta} *\left|\zeta^{\#} \phi\right|(x) \leq \sup _{y \in B_{\delta}(x)}\left|\zeta^{\#} \phi(y)\right| \leq C \sup _{y \in B_{\delta}(x)}|\phi(\zeta(y))| .
$$

With (6.37) this implies that

$$
\left\|\frac{\phi_{\varepsilon}^{1}}{f_{\varepsilon}^{2}}\right\|_{\infty} \leq C\left\|\frac{\phi}{\rho}\right\|_{\infty}
$$

To finish the proof of (6.18), note that $\left\|\zeta^{\#} \phi\right\|_{\infty} \leq C\|\phi\|_{\infty} \leq C\|\phi / \rho\|_{L^{\infty}(\mathcal{D})}$, and so

$$
\left\|\nabla \phi_{\varepsilon}^{1}\right\|_{\infty}=\left\|\left(\nabla \omega_{\delta}\right) *\left(\zeta^{\#} \phi\right)\right\|_{\infty} \leq\left\|\nabla \omega_{\delta}\right\|_{1}\left\|\zeta^{\#} \phi\right\|_{\infty} \leq C \delta^{-1}\left\|\frac{\phi}{\rho}\right\|_{\infty} .
$$

4. To estimate $\phi_{\varepsilon}^{2}$, note that by the mean value theorem,

$$
\left|\phi_{\varepsilon}^{2}\right|(x) \leq C \delta \sup _{y \in B_{\delta}(x)}\left|\nabla\left(\zeta^{\#} \phi\right)\right|(y) .
$$

Recall that if $\phi=\phi^{i} \mathrm{~d} x^{i}$ then $\zeta^{\#} \phi(y)=\phi^{i}(\zeta(y)) \zeta_{x_{j}}^{i}(y) \mathrm{d} x^{j}$, where $\zeta^{i}$ is a component of $\zeta$. Thus $\left(\zeta^{\#} \phi\right)_{x_{k}}=$ $\left[\phi_{x_{l}}^{i}(\zeta) \zeta_{x_{k}}^{l} \zeta_{x_{j}}^{i}+\phi^{i}(\zeta) \zeta_{x_{j} x_{k}}^{i}\right] \mathrm{d} x^{j}$. Using this and the bounds (6.15) on the derivatives of $\zeta$,

$$
\left|\nabla\left(\zeta^{\#} \phi\right)\right|(y) \leq\left(|\nabla \zeta|^{2}|\nabla \phi|+\left|\nabla^{2} \zeta\right||\phi|\right)(\zeta(y)) \leq C\left(|\nabla \phi|+\frac{|\phi|}{\rho}\right)(\zeta(y)) .
$$

Again using (6.37), it follows that

$$
\frac{\left|\phi_{\varepsilon}^{2}\right|}{f_{\varepsilon}^{2}}(x) \leq C \delta \sup _{y \in B_{\delta}(x)} \frac{\left|\nabla \zeta^{\#} \phi\right|}{\rho}(y) \leq C \delta\left(\left\|\frac{\phi}{\rho^{2}}\right\|_{\infty}+\left\|\frac{\nabla \phi}{\rho}\right\|_{\infty}\right)
$$

In addition,

and so the estimate

$$
\left|\nabla \phi_{\varepsilon}^{2}\right|(x) \leq\left|\nabla\left(\zeta^{\#} \phi\right)\right|(x)+\sup _{y \in B_{\delta}(x)}\left|\nabla\left(\zeta^{\#} \phi\right)\right|(y) \leq 2 \sup _{y \in B_{\delta}(x)}\left|\nabla\left(\zeta^{\#} \phi\right)\right|(y)
$$

$$
\left\|\nabla \phi_{\varepsilon}^{2}\right\|_{\infty} \leq C\left\|\frac{\nabla \phi_{\varepsilon}^{2}}{\rho}\right\|_{\infty} \leq C\left(\left\|\frac{\phi}{\rho^{2}}\right\|_{\infty}+\left\|\frac{\nabla \phi}{\rho}\right\|_{\infty}\right)
$$

follows from the last inequality in (6.38), thereby establishing (6.19).

5. Finally, note that $\phi_{\varepsilon}^{3}$ is supported in $\mathcal{D} \backslash \mathcal{D}_{4 \delta}$, a set whose Lebesgue measure is bounded by $C \delta$, so $\left\|\frac{\mathrm{d} \phi_{\varepsilon}^{3}}{f_{\varepsilon}^{2}}\right\|_{4} \leq C \delta^{1 / 4}\left\|\frac{\mathrm{d} \phi_{\varepsilon}^{3}}{f_{\varepsilon}^{2}}\right\|_{\infty}$. And

$$
\left\|\frac{\mathrm{d} \phi_{\varepsilon}^{3}}{f_{\varepsilon}^{2}}\right\|_{\infty} \leq\left\|\frac{\mathrm{d} \phi}{f_{\varepsilon}^{2}}\right\|_{\infty}+\left\|\frac{\mathrm{d} \zeta^{\#} \phi}{f_{\varepsilon}^{2}}\right\|_{\infty} \leq C\left\|\frac{\mathrm{d} \phi}{f_{\varepsilon}^{2}}\right\|_{\infty} .
$$

The last estimate is obtained as above: using the fact that $\mathrm{d} \zeta^{\#} \phi=\zeta^{\#} \mathrm{~d} \phi$ is supported in $\mathcal{D}_{2 \delta}$ and (6.13) and (6.16),

at every $x$ in the support of $\phi_{\varepsilon}^{3}$.

$$
\left|\frac{\zeta^{\#} \mathrm{~d} \phi}{f_{\varepsilon}^{2}}\right|(x) \leq C \frac{|\mathrm{d} \phi|(\zeta(x))}{\rho(x)} \leq C \frac{|\mathrm{d} \phi|}{\rho}(\zeta(x)) \leq C\left\|\frac{\mathrm{d} \phi}{\rho}\right\|_{\infty} \leq C\left\|\frac{\mathrm{d} \phi}{f_{\varepsilon}^{2}}\right\|_{\infty}
$$




\section{VORTEX-FREE GLOBAL MINIMIZERS}

The main result of this section proves that minimizers of $G_{\Omega_{\varepsilon}}^{\varepsilon}$ are asymptotically vortex-free when for the corresponding values of $\Omega$, the minimizer of $E_{\Omega}$ is vortex-free. It would be interesting to know whether for fixed, small $\varepsilon$, a minimizer $u_{\varepsilon}$ is vortex-free (i.e., can be written in the form $u_{\varepsilon}=f e^{i S}$ with $f, S$ real-valued).

The proof is more or less a routine application of the $\Gamma$-limit result established in the previous section, with the one difficulty that one does not know at the outset the scale of the energy, which in this case is encoded in the constants $m_{\varepsilon}$.

Theorem 3. Assume that $\Omega<\Omega_{1}$ (as defined in (3.12)) and that $\Omega_{\varepsilon}$ is related to $\Omega$ as in (6.6). Let $u_{\varepsilon}$ minimize $G_{\Omega_{\varepsilon}}^{\varepsilon}$ in $H_{0}^{1}(\mathcal{D} ; \mathbb{C})$, and define $v_{\varepsilon}=u_{\varepsilon} / \eta_{\varepsilon}$, where $\eta_{\varepsilon}$ is the vortex-free profile defined in $(6.4)$. Then $\star J v_{\varepsilon} \rightarrow 0$ in the sense of distributions as $\varepsilon \rightarrow 0$.

Proof. First, it follows from Lemma 1 that $G_{\Omega_{\varepsilon}}^{\varepsilon}$ is bounded below and that for fixed $\varepsilon$, $G_{\Omega_{\varepsilon}}^{\varepsilon}$ is coercive $\mathrm{n}$ $H^{1}$, and so standard arguments show that a minimizer $u_{\varepsilon}$ exists. Clearly $G_{\Omega_{\varepsilon}}^{\varepsilon}\left(u_{\varepsilon}\right) \leq G_{\Omega_{\varepsilon}}^{\varepsilon}\left(\eta_{\varepsilon}\right)$, so we can use Lemma 11, (6.29), Lemma 13 and Lemma 1 to estimate

$$
\begin{aligned}
H_{e}\left(v_{\varepsilon}\right) & \leq\left|I_{\varepsilon}\left(v_{\varepsilon}\right)\right|+\left|J_{\varepsilon}\left(v_{\varepsilon}\right)\right| \\
& \leq C \varepsilon \Omega_{\varepsilon}{ }^{2} H_{\varepsilon}\left(v_{\varepsilon}\right)^{1 / 2}+C\left\|f_{\varepsilon}\right\|_{\infty} \Omega_{\varepsilon} \int_{\mathcal{D}}\left|j v_{\varepsilon}\right| \\
& \leq C \varepsilon \Omega_{\varepsilon}{ }^{2} H_{\varepsilon}\left(v_{\varepsilon}\right)^{1 / 2}+C|\ln \varepsilon|^{2} .
\end{aligned}
$$

It follows that $H_{\varepsilon}\left(v_{\varepsilon}\right) \leq C|\ln \varepsilon|^{2}$, so that the hypotheses of Theorem 2 are satisfied if $m_{\varepsilon}$ is defined by $m_{\varepsilon}:=$ $\max \left\{1, H_{\varepsilon}\left(v_{\varepsilon}\right) /|\ln \varepsilon|\right\}$. Thus there exists $J \in \mathcal{M}_{1, \rho}(\mathcal{D})$ such that, after passing to a subsequence if necessary, $\star \frac{J v_{\varepsilon}}{m_{\varepsilon}} \rightarrow J$ in the sense of distributions, and

$$
0 \geq \liminf \frac{1}{m_{\varepsilon}|\ln \varepsilon|}\left(G_{\Omega_{\varepsilon}}^{\varepsilon}\left(u_{\varepsilon}\right)-G_{\Omega_{\varepsilon}}^{\varepsilon}\left(\eta_{\varepsilon}\right)\right) \geq E_{\Omega}(J)
$$

This implies that $J=0$, since if $J$ is nontrivial, it would follow from the above that $E_{\Omega^{\prime}}(J)<0$ for all $\Omega^{\prime}>\Omega$, which is impossible in view of the assumption that $\Omega<\Omega_{1}$ and the definition (3.12) of $\Omega_{1}$.

To finish the proof it is only necessary to demonstrate that $m_{\varepsilon}=1$ for all $\varepsilon$ sufficiently small. If this is not the case, then $m_{\varepsilon}|\ln \varepsilon|=H_{\varepsilon}\left(v_{\varepsilon}\right)$, leading to a contradiction:

$$
\begin{aligned}
0 & \geq \liminf \frac{1}{m_{\varepsilon}|\ln \varepsilon|}\left(G_{\Omega_{\varepsilon}}^{\varepsilon}\left(u_{\varepsilon}\right)-G_{\Omega_{\varepsilon}}^{\varepsilon}\left(\eta_{\varepsilon}\right)\right) \\
& =\liminf \frac{1}{m_{\varepsilon}|\ln \varepsilon|}\left(H_{\varepsilon}\left(v_{\varepsilon}\right)+I_{\varepsilon}\left(v_{\varepsilon}\right)+J_{\varepsilon}\left(v_{\varepsilon}\right)\right) \quad \text { by Lemma } 11 \\
& =1 \quad \text { by }(6.29) \text { and (6.30), since } J=0 .
\end{aligned}
$$

\section{Local Minimizers of the Gross-Pitaevsky ENERGy}

The main result of this section is

Theorem 4. Suppose that $M \subset \pi \stackrel{\circ}{\mathcal{R}}_{1, \rho}$ is a $\mathbf{F}_{p, \rho, K}$-local minimizing set for $E_{\Omega}$, for some compact $K \subset \mathcal{D}$ and some $\Omega>0$. Assume that $\Omega_{\varepsilon}$ is a sequence such that $\lim _{\varepsilon \rightarrow 0} \frac{\Omega_{\varepsilon}}{\mid \ln \varepsilon}=\Omega\left(1+\left(a_{1} / a_{2}\right)^{2}\right)$, as in the hypotheses of Theorem 2 .

Then there exists $\sigma, \varepsilon_{0}>0$ (depending on $M, \Omega$ and $\mathcal{D}$ ) such that for every $\varepsilon<\varepsilon_{0}$, $G_{\Omega_{\varepsilon}}^{\varepsilon_{\varepsilon}}$ attains its minimum in the set ${ }^{5}$

$$
\mathcal{A}_{\varepsilon}:=\left\{u \in H_{0}^{1}(\mathcal{D} ; \mathbb{C}): \min _{S \in M} \mathbf{F}_{p, \rho, K}\left(S-\widetilde{J}_{\varepsilon} v_{\varepsilon}\right)<\sigma\right\},
$$

\footnotetext{
${ }^{5}$ The proof of the upper bound (6.11) shows that $\mathcal{A}_{\varepsilon}$ is nonempty for small $\varepsilon$.
} 
for

$$
v_{\varepsilon}:=u / \eta_{\varepsilon}, \quad \widetilde{J}_{\varepsilon} v_{\varepsilon}:=\zeta_{\#} \omega_{\delta} *\left(\star J v_{\varepsilon}\right)
$$

as in (6.17), with $\delta=\varepsilon^{\gamma}$ and $\gamma$ as in Theorem 2. Moreover, $\mathcal{A}_{\varepsilon}$ is open in $H_{0}^{1}(\mathcal{D} ; \mathbb{C})$, so the minimizer of $G_{\Omega_{\varepsilon}}^{\varepsilon}$ in $\mathcal{A}_{\varepsilon}$ is a local minimizer of $G_{\Omega_{\varepsilon}}^{\varepsilon_{1}}$ and a solution of the Euler-Lagrange equations. Finally, if $u_{\varepsilon}^{*}$ denotes a local minimizer of $G_{\Omega_{\varepsilon}}$ in $\mathcal{A}_{\varepsilon}$ and $v_{\varepsilon}{ }^{*}=u_{\varepsilon} / \eta_{\varepsilon}$, then $\min _{S \in M} \mathbf{F}_{p, \rho, K}\left(S-\widetilde{J}_{\varepsilon} v_{\varepsilon}{ }^{*}\right) \rightarrow 0$ as $\varepsilon \rightarrow 0$. In particular, if $M$ consists of a single, $\mathbf{F}_{p, \rho, K}$-local minimizing current $S$, then $\star J v_{\varepsilon}{ }^{*} \rightarrow S$ is the sense of distributions as $\varepsilon \rightarrow 0$.

In view of Theorem 1, this immediately implies the existence, for every ellipsoidal domain $\mathcal{D}$, all $\Omega>\Omega_{0}(\mathcal{D})$, and sufficiently small $\varepsilon$, of large numbers of local minimizers of $E_{\Omega}$. In addition, when the domain $\mathcal{D}$ satisfies the nondegeneracy condition $a_{1} \neq a_{2}$, then for a.e. $\Omega>\Omega_{0}$ and every pair of positive nonnegative integers $k_{1}, k_{2}$, it shows the existence (for $\varepsilon \leq \varepsilon_{0}\left(\Omega, k_{1}, k_{2}, \mathcal{D}\right)$ ) of a local minimizer $u_{\varepsilon}^{*}$ of $E_{\Omega}$ whose vorticity is near the current $T_{\Omega ; k_{1}, k_{2}}$ constructed in Corollary 1 .

The proof is essentially an application of the well-known Kohn-Sternberg scheme [14] for proving the existence of local minimizers using $\Gamma$-convergence, see also for example [16]. To verify that their argument is available here, it is necessary to check that the map $u_{\varepsilon} \mapsto \widetilde{J}_{\varepsilon} v_{\varepsilon}$ is continuous from $H^{1}$ into $\mathcal{M}_{1, \rho}(\mathcal{D})$ with the $\mathbf{F}_{p, \rho, K}$ seminorm, and roughly speaking that $u_{\varepsilon} \mapsto \mathbf{F}_{p, \rho, K}\left(\widetilde{J}_{\varepsilon} v_{\varepsilon}\right)$ is weakly lowersemicontinuous in $H^{1}$. These are carried out in Steps 1 and 2 respectively of the proof.

Proof of Theorem 4. Throughout this proof, $\delta=\varepsilon^{\gamma}$, where $\gamma$ is as in Theorem 2, and $\zeta=\zeta_{\delta}$ and $\omega_{\delta}$ are as defined in Step 1 of the proof of Theorem 2.

1. I first assert that for every $\varepsilon>0$ there exists a constant $C_{\varepsilon}>0$ such that for every $u, \tilde{u} \in H_{0}^{1}(\mathcal{D})$,

$$
\mathbf{F}_{p, \rho, K}\left(\widetilde{J}_{\varepsilon} v_{\varepsilon}-\widetilde{J}_{\varepsilon} \tilde{v}_{\varepsilon}\right) \leq C_{\varepsilon}\|u-\tilde{u}\|_{H^{1}}\left(\|u\|_{H^{1}}+\|\tilde{u}\|_{H^{1}}\right)
$$

where $v_{\varepsilon}=u / \eta_{\varepsilon}$ and $\tilde{v_{\varepsilon}}=\tilde{u} / \eta_{\varepsilon}$. This will use the following fact, which is proved in Lemma 16 in Section 9: for every compact $K \subset \mathcal{D}$, there exists $C_{K}$ such that

$$
\mathbf{F}_{p, \rho, K}(T) \leq C_{K} \mathbf{F}_{\rho}(T)=C_{K} \inf \left\{\mathbf{M}_{\rho}(S): S \in \mathcal{M}_{2, \rho}(\mathcal{D}), \partial S=T\right\}
$$

Since

$$
\widetilde{J}_{\varepsilon} v_{\varepsilon}-\widetilde{J}_{\varepsilon} \tilde{v}_{\varepsilon}=\frac{1}{2} \zeta_{\#} \omega_{\delta} *\left(\partial\left(\star j v_{\varepsilon}-\star j \tilde{v}_{\varepsilon}\right)\right)=\frac{1}{2} \partial\left(\zeta_{\#} \omega_{\delta} *\left(\star j v_{\varepsilon}-\star j \tilde{v}_{\varepsilon}\right)\right)
$$

we can use (8.3) to estimate

$$
\begin{aligned}
\mathbf{F}_{p, \rho, K}\left(\widetilde{J}_{\varepsilon} v_{\varepsilon}-\widetilde{J}_{\varepsilon} \tilde{v}_{\varepsilon}\right) & \leq C \mathbf{M}_{\rho}\left(\zeta_{\#} \omega_{\delta} *\left(\star j v_{\varepsilon}-\star j \tilde{v}_{\varepsilon}\right)\right) \\
& \leq C \sup \left\{\zeta_{\#} \omega_{\delta} *\left(\star j v_{\varepsilon}-\star j \tilde{v}_{\varepsilon}\right)(\phi):\left\|\frac{\phi}{\rho}\right\|_{\infty} \leq 1\right\} \\
& =C \sup \left\{\star\left(j v_{\varepsilon}-j \tilde{v}_{\varepsilon}\right)\left(\phi_{\varepsilon}^{1}\right):\left\|\frac{\phi}{\rho}\right\|_{\infty} \leq 1\right\} .
\end{aligned}
$$

using the notation $\phi_{\varepsilon}^{1}:=\omega_{\delta} * \zeta^{\#} \phi$ of Section 6 . And

$$
\begin{aligned}
\star\left(j v_{\varepsilon}-j \tilde{v}_{\varepsilon}\right)\left(\phi_{\varepsilon}^{1}\right) & \leq \int_{\mathcal{D}}\left|\phi_{\varepsilon}^{1}\right|\left|j v_{\varepsilon}-j \tilde{v}_{\varepsilon}\right| \\
& \leq\left\|\frac{\phi_{\varepsilon}^{1}}{f_{\varepsilon}^{2}}\right\|_{L^{\infty}}\left\|f_{\varepsilon}^{2}\left(j v_{\varepsilon}-j \tilde{v}_{\varepsilon}\right)\right\|_{L^{1}} .
\end{aligned}
$$

Note that $f_{\varepsilon}^{2} j v_{\varepsilon}=j\left(f_{\varepsilon} v_{\varepsilon}\right)=j\left(e^{-i \Omega_{\varepsilon} S_{0}} u\right)=j u-|u|^{2} \Omega_{\varepsilon} \mathrm{d} S_{0}$, and similarly for $j \tilde{v}_{\varepsilon}$. Also, in view of (6.18), $\left\|\phi_{\varepsilon}^{1} / f_{\varepsilon}^{2}\right\|_{\infty} \leq C\|\phi / \rho\|_{\infty}$. Thus the above inequalities yield

$$
\mathbf{F}_{p, \rho, K}\left(\widetilde{J}_{\varepsilon} v_{\varepsilon}-\widetilde{J}_{\varepsilon} \tilde{v}_{\varepsilon}\right) \leq C_{\varepsilon}\left(\|j u-j \tilde{u}\|_{L^{1}}+\left\||u|^{2}-|\tilde{u}|^{2}\right\|_{L^{1}}\right),
$$


and from this one can easily deduce (8.2).

It follows from $(8.2)$ that for every $\varepsilon, \mathcal{A}_{\varepsilon}$ is open in $H_{0}^{1}(\mathcal{D} ; \mathbb{C})$.

2. In this step I will show that for every fixed $\varepsilon>0$,

$$
\mathcal{A}_{\varepsilon}{ }^{*}:=\left\{u \in H_{0}^{1}(\mathcal{D} ; \mathbb{C}): \min _{S \in M} \mathbf{F}_{p, \rho, K}\left(S-\widetilde{J}_{\varepsilon} v_{\varepsilon}\right) \leq \sigma, v_{\varepsilon}=\frac{u}{\eta_{\varepsilon}}\right\}
$$

is weakly closed in $H_{0}^{1}(\mathcal{D} ; \mathbb{C})$. To do this, suppose that $u_{k} \in \mathcal{A}_{\varepsilon}{ }^{*}$ and $u_{k} \rightarrow u_{\infty}$ weakly in $H_{0}^{1}$. I must show that $u_{\infty} \in \mathcal{A}_{\varepsilon}{ }^{*}$. To do this, I first claim there exists $C_{\varepsilon}$ such that for any $u \in H_{0}^{1}(\mathcal{D})$

$$
\int_{\mathcal{D}_{\delta}} f_{\varepsilon}^{2} \frac{\left|\nabla v_{\varepsilon}\right|^{2}}{2}+\frac{f_{\varepsilon}^{4}}{4 \varepsilon^{2}}\left(\left|v_{\varepsilon}\right|^{2}-1\right)^{2} \mathrm{~d} x \leq C_{\varepsilon}\left(1+\|u\|_{H_{0}^{1}}^{4}\right), \quad v_{\varepsilon}=u / \eta_{\varepsilon} \text { as usual. }
$$

Since $v_{\varepsilon}=u e^{-i \Omega_{\varepsilon} S_{0}} / f_{\varepsilon}$, with $S_{0}$ a fixed, smooth function,

$$
\int_{\mathcal{D}_{\delta}} f_{\varepsilon}^{2}\left|\nabla v_{\varepsilon}\right|^{2} \leq C_{\varepsilon} \int_{\mathcal{D}_{\delta}}\left(\frac{\left|\nabla f_{\varepsilon}\right|^{2}}{f_{\varepsilon}^{2}}|u|^{2}+|\nabla u|^{2}+|u|^{2}\right) \mathrm{d} x
$$

By elliptic regularity, $\left|\nabla f_{\varepsilon}\right| \leq C_{\varepsilon}$ on $\mathcal{D}_{\delta}$, and Lemma 13 implies that $f_{\varepsilon} \geq C_{\varepsilon}^{-1}$ on $\mathcal{D}_{\delta}$, and so

$$
\int_{\mathcal{D}_{\delta}} f_{\varepsilon}^{2}\left|\nabla v_{\varepsilon}\right|^{2} \leq C_{\varepsilon} \int_{\mathcal{D}_{\delta}}\left(|u|^{2}+|\nabla u|^{2}\right) \mathrm{d} x \leq C_{\varepsilon}\|u\|_{H_{0}^{1}}^{2} .
$$

Similarly

$$
\int_{\mathcal{D}_{\delta}} f_{\varepsilon}^{4}\left(\left|v_{\varepsilon}\right|^{2}-1\right)^{2} \leq C \int_{\mathcal{D}_{\delta}}\left(\left|u_{\varepsilon}\right|^{4}+1\right) \mathrm{d} x \leq C_{\varepsilon}\left(1+\|u\|_{H_{0}^{1}}^{4}\right)
$$

using the Sobolev embedding. The two inequalities establish (8.5).

For any smooth 1-form $\phi$ with compact support, (8.5) together with (6.21), (6.22) shows that

$$
\star J v_{\varepsilon}\left(\phi_{\varepsilon}^{1}\right) \leq C_{\varepsilon}\left(1+\|u\|_{H_{0}^{1}}^{4}\right)\left(\left\|\frac{\phi_{\varepsilon}^{1}}{f_{\varepsilon}^{2}}\right\|_{\infty}+\varepsilon^{\gamma}\left(1+\left\|\nabla \phi_{\varepsilon}^{1}\right\|_{\infty}\right)\left(1+\|\phi\|_{\infty}\right)\right) .
$$

Now the weak convergence $u_{k} \rightarrow u$ implies that $\left\|u_{k}\right\|_{H_{0}^{1}}$ is uniformly bounded. Thus, writing $v_{k, \varepsilon}:=u_{k} / \eta_{\varepsilon},(6.18)$ allows us to deduce that if $\|\phi / \rho\|_{\infty} \leq 1$, then

$$
\begin{aligned}
\widetilde{J}_{\varepsilon} v_{k, \varepsilon}(\phi) & =\star J v_{k, \varepsilon}\left(\phi_{\varepsilon}^{1}\right) \\
& \leq C_{\varepsilon}\left(1+\left\|u_{k}\right\|_{H_{0}^{1}}\right)\left(\left\|\frac{\phi_{\varepsilon}^{1}}{f_{\varepsilon}^{2}}\right\|_{\infty}+\varepsilon^{\gamma}\left(1+\left\|\nabla \phi_{\varepsilon}^{1}\right\|_{\infty}\right)\left(1+\|\phi\|_{\infty}\right)\right) \\
& \leq C_{\varepsilon} .
\end{aligned}
$$

Thus $\mathbf{M}_{\rho}\left(\widetilde{J}_{\varepsilon} v_{k, \varepsilon}\right)=\sup \left\{\widetilde{J}_{\varepsilon} v_{k, \varepsilon}(\phi):\|\phi / \rho\|_{\infty} \leq 1\right\}$ is bounded independent of $k$, and so Lemma 2 implies that there exists some current $J \in \mathcal{M}_{1, \rho}(\mathcal{D})$ such that, after passing to a subsequence,

$$
\mathbf{F}_{p, \rho, K}\left(\widetilde{J}_{\varepsilon} v_{k, \varepsilon}-J\right) \rightarrow 0
$$

as $k \rightarrow \infty$. Since $u_{k} \in \mathcal{A}_{\varepsilon}{ }^{*}$ for all $k$, it follows that

$$
\min _{S \in M} \mathbf{F}_{p, \rho, K}(S-J) \leq \sigma .
$$


I now claim that

$$
J=\widetilde{J}_{\varepsilon} v_{\infty, \varepsilon}, \quad v_{\infty, \varepsilon}:=u_{\infty} / \eta_{\varepsilon}
$$

To establish this, it suffices to prove that $\widetilde{J}_{\varepsilon} v_{k, \varepsilon} \rightarrow \widetilde{J}_{\varepsilon} v_{\infty, \varepsilon}$ in the sense of distributions. This follows by observing that, since $S_{0}$ and $f_{\varepsilon}$ are fixed functions, smooth on $\mathcal{D}_{\delta}, v_{k, \varepsilon} \rightarrow v_{\infty, \varepsilon}:=u_{\infty} / \eta_{\varepsilon}$ weakly in $H^{1}\left(\mathcal{D}_{\delta}\right)$, and so standard facts about weak convergence of determinants imply that $J v_{k, \varepsilon} \rightarrow J v_{\infty, \varepsilon}$ in the sense of distributions on $\mathcal{D}_{\delta}$. Because the map $J v_{\varepsilon} \mapsto \widetilde{J}_{\varepsilon} v_{\varepsilon}=\zeta_{\#} \omega_{\delta} * J v_{\varepsilon}$ is linear and depends only on the behavior of $J v_{\varepsilon}$ in $\mathcal{D}_{\delta}$, this shows that $\widetilde{J}_{\varepsilon} v_{k, \varepsilon} \rightarrow \widetilde{J}_{\varepsilon} v_{\infty, \varepsilon}$ in the sense of distributions as desired, and hence proves (8.7).

Combining (8.6) and (8.7), one finds that $u_{\infty} \in \mathcal{A}_{\varepsilon}{ }^{*}$, and so $\mathcal{A}_{\varepsilon}{ }^{*}$ is weakly closed.

3. Now let $u_{\varepsilon}$ minimize $G_{\Omega_{\varepsilon}}^{\varepsilon}$ in $\mathcal{A}_{\varepsilon}{ }^{*}$; indeed, $G_{\Omega_{\varepsilon}}^{\varepsilon}$ is bounded below, as a result of Lemma 1, and it is standard that such a functional is weakly lower semicontinuous in $H^{1}$, and so the existence of a minimizer in $\mathcal{A}_{\varepsilon}{ }^{*}$ follows from the previous step.

In this step I show that $H_{\varepsilon}\left(v_{\varepsilon}\right) \leq C|\ln \varepsilon|$ with a uniform constant $C$ for $\varepsilon \in(0,1 / 2)$. According to Theorem 2, this will imply that $\left\{\widetilde{J}_{\varepsilon} v_{\varepsilon}\right\}$ is precompact with respect to the $\mathbf{F}_{p, \rho, K}$ seminorms for all compact $K \subset \mathcal{D}$.

To do this, first note from Lemma 11 that

$$
H_{\varepsilon}\left(v_{\varepsilon}\right) \leq\left(G_{\Omega_{\varepsilon}}^{\varepsilon}\left(u_{\varepsilon}\right)-G_{\Omega_{\varepsilon}}^{\varepsilon}\left(\eta_{\varepsilon}\right)\right)+\left|I_{\varepsilon}\left(v_{\varepsilon}\right)\right|+\left|J_{\varepsilon}\left(v_{\varepsilon}\right)\right|
$$

we can select $J \in M$ and, by Theorem 2 , construct a sequence $\hat{u}_{\varepsilon}$ such that for $\lim _{\varepsilon} \frac{1}{|\ln \varepsilon|}\left(G_{\Omega_{\varepsilon}}^{\varepsilon}\left(\hat{u}_{\varepsilon}\right)-G_{\Omega_{\varepsilon}}^{\varepsilon}\left(\eta_{\varepsilon}\right)\right)=$ $E_{\Omega}(J)$, and

$$
\mathbf{F}_{p, \rho, K}\left(\widetilde{J}_{\varepsilon} v_{\varepsilon}-J\right) \rightarrow 0, \quad \hat{v}_{\varepsilon}=\hat{u}_{\varepsilon} / \eta_{\varepsilon}
$$

Thus $\hat{u}_{\varepsilon} \in \mathcal{A}_{\varepsilon}$ for all $\varepsilon$ sufficiently small, and because $G_{\Omega_{\varepsilon}}^{\varepsilon}\left(u_{\varepsilon}\right) \leq G_{\Omega_{\varepsilon}}^{\varepsilon}\left(\hat{u}_{\varepsilon}\right)$, it follows that

$$
\liminf _{\varepsilon \rightarrow 0} \frac{1}{|\ln \varepsilon|}\left(G_{\Omega_{\varepsilon}}^{\varepsilon}\left(u_{\varepsilon}\right)-G_{\Omega_{\varepsilon}}^{\varepsilon}\left(\eta_{\varepsilon}\right)\right) \leq E_{\Omega}(J)
$$

Next, use (6.29) to estimate

$$
\left|I_{\varepsilon}\left(v_{\varepsilon}\right)\right| \leq C \varepsilon|\ln \varepsilon|^{2} \sqrt{H_{\varepsilon}\left(v_{\varepsilon}\right)} \leq C \sqrt{\varepsilon H_{\varepsilon}\left(v_{\varepsilon}\right)} \leq C+\varepsilon H_{\varepsilon}\left(v_{\varepsilon}\right) .
$$

And using (6.36) ${ }^{6}$ and Lemma 5,

$$
J_{\varepsilon}\left(v_{\varepsilon}\right)=\frac{-\Omega_{\varepsilon}}{1+\left(a_{1} / a_{2}\right)^{2}} \widetilde{J}_{\varepsilon} v_{\varepsilon}\left(\rho^{2} \mathrm{~d} x^{3}\right)+O\left(\varepsilon^{\alpha}\right) \leq C|\ln \varepsilon|\left(\mathbf{F}_{p, \rho, K}\left(\widetilde{J}_{\varepsilon} v_{\varepsilon}\right)+1\right)
$$

for some $\alpha>0$. But the definition of $\mathcal{A}_{\varepsilon}$ and the fact that $M$, as a locally minimizing set, is $\mathbf{F}_{p, \rho, K}$-compact, together imply that $\mathbf{F}_{p, \rho, K}\left(\widetilde{J}_{\varepsilon} v_{\varepsilon}\right) \leq C$ for all $u_{\varepsilon} \in \mathcal{A}_{\varepsilon}$. Thus $\left|J_{\varepsilon}\left(v_{\varepsilon}\right)\right| \leq C|\ln \varepsilon|$, and this with (8.9) and (8.10) shows that $H_{\varepsilon}\left(v_{\varepsilon}\right) \leq C|\ln \varepsilon|$.

4. I now claim that for $\varepsilon, \sigma$ sufficiently small, $u_{\varepsilon}$ belongs to $\mathcal{A}_{\varepsilon}$.

Suppose toward a contradiction that this does not hold. Then there exists a subsequence, which for convenience I continue to write as $v_{\varepsilon}$, such that

$$
\min _{S \in M} \mathbf{F}_{p, \rho, K}\left(S-\widetilde{J}_{\varepsilon} v_{\varepsilon}\right)=\sigma, \quad v_{\varepsilon}=\frac{u}{\eta_{\varepsilon}}
$$

Upon passing to a further subsequence if necessary, we can also assume that there exists some limiting current $J^{\prime} \in \pi \mathcal{R}_{1, \rho}$ such that $J v_{\varepsilon} \rightarrow J^{\prime}$ in the sense of distributions, and $\mathbf{F}_{p, \rho, K}\left(J^{\prime}-\widetilde{J}_{\varepsilon} v_{\varepsilon}\right) \rightarrow 0$. It follows that

$$
\min _{S \in M} \mathbf{F}_{p, \rho, K}\left(S-J^{\prime}\right)=\sigma
$$

\footnotetext{
${ }^{6}$ Note that (6.36) does not require any assumed bounds such as (6.7) on $H_{\varepsilon}\left(v_{\varepsilon}\right)$, and uses only the assumption (6.6), so the argument is not circular here.
} 
And (6.10), (8.9) imply that

$$
E_{\Omega}(J) \geq \liminf _{\varepsilon \rightarrow 0} \frac{1}{|\ln \varepsilon|}\left(G_{\Omega_{\varepsilon}}^{\varepsilon}\left(u_{\varepsilon}\right)-G_{\Omega_{\varepsilon}}^{\varepsilon}\left(\eta_{\varepsilon}\right)\right) \geq E_{\Omega}\left(J^{\prime}\right) .
$$

However, in view of the definition of a $\mathbf{F}_{p, \rho, K}$-local minimizing set, if $\sigma$ is small enough then (8.11) implies that $E_{\Omega}(J)<E_{\Omega}\left(J^{\prime}\right)$, contradicting (8.12).

5. To see that $\min _{S \in M} \mathbf{F}_{p, \rho, K}\left(S-\widetilde{J}_{\varepsilon} v_{\varepsilon}\right) \rightarrow 0$, it suffices to note that one can replace $\sigma$ by any $0<\sigma^{\prime}<\sigma$ in the above arguments.

And in the case when $M$ contains a single current $S$, the above arguments show that $\mathbf{F}_{p, \rho, K}\left(S-\widetilde{J}_{\varepsilon} v_{\varepsilon}\right) \rightarrow 0$ as $\varepsilon \rightarrow 0$, for every compact $K \subset \mathcal{D}$. In view of (6.9), this shows that $\star J v_{\varepsilon} \rightarrow S$ in the sense of distributions.

\section{ApPendix: SOME TECHNICAL LEMmas}

In this appendix I collect the proofs of some technical results that are not central to the main argument.

First I give the

Proof of Lemma 2.

1. Given $\left\{R_{k}\right\} \subset \dot{\mathcal{M}}_{1, \rho}\left(\mathcal{D}_{0}\right)$ such that $\mathbf{M}_{\rho}\left(R_{k}\right) \leq C$, let $\left\{u_{k}\right\} \subset B V_{\text {loc }}\left(\mathcal{D}_{0}\right)$ be functions such that $\star \mathrm{d} u_{k}=R_{k}$, using notation of Lemma 4 . I normalize $u_{k}$ by requiring that $\int_{V} u_{k} \mathrm{~d} x=0$ for all $k$, where $V \subset \mathcal{D}_{0}$ is an open subset that will be specified later. From (3.15) I deduce that

$$
\int_{\mathcal{D}_{0}} \rho\left|\nabla u_{k}\right| \leq C \quad \text { for all } k \text {. }
$$

I will show that there exists a function $u \in B V_{\text {loc }}\left(\mathcal{D}_{0}\right)$ with $\star \mathrm{d} u \in \dot{\mathcal{M}}_{1, \rho}\left(\mathcal{D}_{0}\right)$ such that after passing to a subsequence,

$$
\int_{\mathcal{D}_{0}} \rho\left|u_{k}-u\right| \rightarrow 0 \quad \text { as } k \rightarrow \infty
$$

In view of (3.15), this will prove that $\mathbf{F}_{\rho}\left(R_{k}-R\right) \rightarrow 0$ for $R=\star \mathrm{d} u$.

2. To prove (9.2), I first claim that there exists a constant $C$ such that

$$
\int_{\mathcal{D}_{0}}|w| \leq C \int_{\mathcal{D}_{0}} \rho|\nabla w|
$$

for all $w \in B V_{\text {loc }}\left(\mathcal{D}_{0}\right)$ such that $\int_{V} w=0$. To do this, first note that since $\rho=0$ and $\nabla \rho \neq 0$ on $\partial \mathcal{D}_{0}$, there exists a constant $C$ such that, if $\sigma_{0}$ is chosen to be sufficiently small, then

$$
\nabla \cdot(\rho \nabla \rho)=\rho \Delta \rho+|\nabla \rho|^{2} \geq C^{-1} \text { whenever } \rho<2 \sigma_{0} .
$$

Thus for every $\sigma \in\left(\sigma_{0}, 2 \sigma_{0}\right)$,

$$
\begin{aligned}
\int_{\left\{x \in \mathcal{D}_{0}: \rho(x) \leq \sigma\right\}}|w| & \leq C \int_{\left\{x \in \mathcal{D}_{0}: \rho(x) \leq \sigma\right\}} \nabla \cdot(\rho \nabla \rho)|w| \\
& =-C \int_{\left\{x \in \mathcal{D}_{0}: \rho(x)<\sigma\right\}} \rho \nabla \rho \cdot \nabla w \frac{w}{|w|}+C \int_{\left\{x \in \mathcal{D}_{0}: \rho(x)=\sigma\right\}}|w| \rho \nabla \rho \cdot \nu .
\end{aligned}
$$


Averaging over $\sigma \in\left(\sigma_{0}, 2 \sigma_{0}\right)$ yields

$$
\begin{aligned}
\int_{\left\{x \in \mathcal{D}_{0}: \rho(x) \leq \sigma_{0}\right\}}|w| & \leq \frac{1}{\sigma_{0}} \int_{\sigma_{0}}^{2 \sigma_{0}} \int_{\left\{x \in \mathcal{D}_{0}: \rho(x) \leq \sigma\right\}}|w| \mathrm{d} x \mathrm{~d} \sigma \\
& \leq C \int_{\left\{x \in \mathcal{D}_{0}: \rho(x) \leq 2 \sigma_{0}\right\}} \rho|\nabla w|+C \int_{\left\{x \in \mathcal{D}_{0}: \sigma_{0} \leq \rho(x) \leq 2 \sigma_{0}\right\}}|w| .
\end{aligned}
$$

Thus to prove (9.3) it suffices to show that

$$
\int_{\left\{x \in \mathcal{D}_{0}: \rho(x) \geq \sigma_{0}\right\}}|w| \leq C \int_{\mathcal{D}_{0}} \rho|\nabla w| .
$$

To do this, let $V=\left\{x \in \mathcal{D}_{0}: \rho(x) \geq \sigma_{0}\right\}$. Then since $\int_{V} w=0$, Poincaré's inequality yields

$$
\int_{V}|w| \leq C \int_{V}|\nabla w| \leq C \int_{V} \rho|\nabla w|
$$

establishing (9.3).

3. From (9.3) and (9.1) it follows that $\int_{\mathcal{D}_{0}}\left|u_{k}\right| \leq C$, and from this and (9.1) it follows that

$$
\int_{\mathcal{D}_{0}}\left|\rho u_{k}\right|+\left|\nabla\left(\rho u_{k}\right)\right| \leq C
$$

Then Rellich's theorem implies that $\left\{\rho u_{k}\right\}$ is precompact in $L^{1}$, and so after passing to a subsequence there exists a function $u$ such that $u_{k} \rightarrow u$ in $L_{\text {loc }}^{1}\left(\mathcal{D}_{0}\right)$ and $\rho u_{k} \rightarrow \rho u$ in $L^{1}\left(\mathcal{D}_{0}\right)$, that is, such that (9.2) holds. To verify that $\star d u \in \dot{\mathcal{M}}_{1, \rho}\left(\mathcal{D}_{0}\right)$, note that

$$
\int_{\mathcal{D}_{0}} \rho|\nabla u|=\sup _{W \subset \subset \mathcal{D}_{0}} \int_{W} \rho|\nabla u| \leq \sup _{W \subset \subset \mathcal{D}_{0}} \liminf \int_{W} \rho\left|\nabla u_{k}\right| \leq C
$$

using the lower semicontinuity of the $B V$ seminorm with respect to $L^{1}$ convergence (see for example [9], Chap. 5).

4. The second claim of the lemma, i.e. the $\mathbf{F}_{p, \rho, K}$ compactmess of $\mathbf{M}_{\rho}$-bounded sequences in $\dot{\mathcal{M}}_{1, \rho}(\mathcal{D})$, follows from the first. Let $\left\{T_{k}\right\}_{k=1}^{\infty} \subset \dot{\mathcal{M}}_{1, \rho}(\mathcal{D})$ be a sequence of currents such that $\mathbf{M}_{\rho}\left(T_{k}\right) \leq C$. First, the currents $T_{k}$ have uniformly bounded mass on every compact subset of $\mathcal{D}$ and satisfy $\partial T_{k}=0$, so compactness in the $\mathbf{F}_{K}$ seminorm for compact $K \subset \mathcal{D}$ is a consequence of a classical result of Federer and Fleming, see [10] 4.2.17. By a diagonal argument, one can find a subsequence (still labelled $T_{k}$ ) and a limiting current $T$ such that $\mathbf{F}_{K}\left(T_{k}-T\right) \rightarrow 0$ for every compact $K \subset \mathcal{D}$.

I claim that $\mathbf{M}_{\rho}(T)<\infty$. To see this, let $K_{n}:=\left\{x \in \mathcal{D}: 2^{-n-1} \leq \rho(x)<2^{-n}\right\}$. Then using the lower semicontinuity of mass with respect to $\mathbf{F}_{K}$ convergence,

$$
\begin{aligned}
\int_{\mathcal{D}} \rho \mathrm{d}\|T\| \leq \sum_{n} 2^{-n} \mathbf{M}_{K_{n}}(T) & \leq \liminf _{k \rightarrow \infty} \sum_{n} 2^{-n} \mathbf{M}_{K_{n}}\left(T_{k}\right) \\
& \leq \liminf _{k \rightarrow \infty} 2 \int_{\mathcal{D}} \rho \mathrm{d}\left\|T_{k}\right\| \leq C .
\end{aligned}
$$

Finally, as is verified in the proof of Lemma $6, \mathbf{M}_{\rho}\left(p_{\#} S\right) \leq \mathbf{M}_{\rho}(S)$ for all $S \in \mathcal{M}_{1, \rho}(\mathcal{D})$, so $\mathbf{M}_{\rho}\left(p_{\#}\left(T_{k}-T\right)\right) \leq C$. It therefore follows the first part of the lemma that $\left\{p_{\#}\left(T_{k}-T\right)\right\}_{k}$ is $\mathbf{F}_{\rho}$-precompact, or equivalently that $\left\{T_{k}-T\right\}_{k}$ is $\mathbf{F}_{p, \rho}$-precompact. However, since $T_{k} \rightarrow T$ in $\mathbf{F}_{K}$ for every compact $K$, it must be that $T_{k}-T$ converges in the $\mathbf{F}_{p, \rho}$ seminorm to 0 . This completes the proof. 
The next lemma provides a decomposition of a 1-current $T$ into a sum of Lipschitz curves. This is known if $T$ has finite mass, and so the point is to prove that such a decomposition remains possible when $T$ has infinite mass (but $\|T\|,\|\partial T\|$ are locally finite.)

\section{Proof of Lemma 3.}

1. In Federer [10] 4.2.25 it is shown that, if $U$ is any open subset of some Euclidean space and $T \in \mathcal{R}_{1}(U)$ is a integral 1-current such that $\mathbf{M}(T), \mathbf{M}(\partial T)<\infty$, then there exist Lipschitz curves $X_{i}$ in $U$ such that $T=\sum T_{X_{i}}$ and

$$
\mathbf{M}_{U}(T)+\mathbf{M}_{U}(\partial T)=\sum \mathbf{M}_{U}\left(T_{i}\right)+\sum \mathbf{M}_{U}\left(\partial T_{i}\right)
$$

This implies (3.14). To see this, note that the identity $T=\sum_{i} T_{X_{i}}$ easily implies that $\|T\|-\sum_{i}\left\|T_{X_{i}}\right\|$ is a nonpositive measure, and similarly $\|\partial T\|-\sum_{i}\left\|\partial T_{X_{i}}\right\|$ is a nonpositive measure. So to prove (3.14), it suffices show that $\mathbf{M}_{U}(T)-\sum \mathbf{M}_{U}\left(T_{i}\right) \geq 0$ and $\mathbf{M}_{U}(\partial T)-\mathbf{M}_{U}\left(\partial T_{i}\right) \geq 0$, and both of these follow from (9.4).

2. Now fix $T$ such that the $\|T\|,\|\partial T\|$ are only locally finite. Let $U_{i}$ be an increasing sequence of open subsets of $U$ such that $\bar{U}_{i} \subset U_{i+1}, \partial U_{i}$ is smooth, and $\partial\left(T\left\lfloor U_{i}\right)\right.$ is a 0-current of finite mass for every $i$, where $T\left\lfloor U_{i}\right.$ denotes the restriction of $T$ to $U_{i}$. This is possible because $\|\partial T\|$ has locally finite mass. Applying the decomposition of step 1 on $U_{1}$, we can write the result as $T\left\lfloor U_{1}=\sum_{j} T_{X_{j}}+\sum_{k} T_{Y_{k}}\right.$, where the analog of (3.14) holds, each $T_{X_{j}}$ is compactly supported within $U_{i}$ and each $T_{Y_{k}}$ has support that intersects $\partial U_{i}$. Now let $T^{(1)}=T-\sum T_{X_{j}}$, and decompose $T^{(1)}$ on $U_{2}$, separating the currents thereby obtained into ones with compact and non-compact support in $U_{2}$. Proceeding inductively, we obtain a decomposition $T=T^{\prime}+\sum T_{X_{i}}$, with

$$
\|T\|=\left\|T^{\prime}\right\|+\sum\left\|T_{X_{i}}\right\| \quad \text { and } \quad\|\partial T\|=\left\|\partial T^{\prime}\right\|+\sum\left\|\partial T_{X_{i}}\right\| \text { as measures }
$$

each $T_{X_{i}}$ has compact support in $U$, and there do not exist any integral currents $T_{1}^{\prime}, T_{2}^{\prime}$ such that $\left\|T^{\prime}\right\|=$ $\left\|T_{1}^{\prime}\right\|+\left\|T_{2}^{\prime}\right\|$ and $\left\|\partial T^{\prime}\right\|=\left\|\partial T_{1}^{\prime}\right\|+\left\|\partial T_{2}^{\prime}\right\|$ as measures, with $T_{1}^{\prime}$ having compact support in $U$.

Now apply the decomposition procedure of step 1 to write $T_{i}^{\prime}:=T^{\prime}\left\lfloor U_{i}\right.$ in the form $\sum_{j=1}^{J(i)} S_{j}^{i}$, where each $S_{j}^{i}$ is the current associated with integration over a Lipschitz curve $X_{j}^{i}$, and (3.14) holds for each $i$. The construction of $T^{\prime}$ implies that $\partial S_{j}^{i} \neq 0$ for every $i, j$, and so for each $i$, the number $J(i)$ of current in the decomposition of $T_{i}^{\prime}$ is bounded by

$$
\sum \mathbf{M}_{U}\left(\partial S_{j}^{i}\right)=\mathbf{M}_{U}\left(\partial T_{i}^{\prime}\right)<\infty
$$

by the choice of $U_{i}$. Now relabel the currents inductively, as follows:

First, let $T_{j}^{1}=S_{j}^{1}, j=1, \ldots, J(1)$. Suppose we are given given $\left\{T_{j}^{i}\right\}_{j=1}^{J^{\prime}(i)} ;$ I will define $\left\{T_{j}^{i+1}\right\}$. Starting with $j=1$, if $S_{1}^{i+1}\left\lfloor U_{i}\right.$ equals zero, then set $T_{J(i)+1}^{i+1}:=S_{1}^{i+1}$; otherwise, $S_{1}^{i+1}\left\lfloor U_{i}\right.$ can be written as a sum of currents $T_{j_{1}}^{i}+\cdots+T_{j_{m}}^{i}$ with $j_{1}<\cdots<j_{m}$. When this holds, relabel $S_{j}^{i+1}=: T_{j_{1}}^{i+1}$ and set $T_{j_{k}}^{i+1}=0$ for $k=2, \ldots, m$. Then continue inductively, relabelling $S_{j}^{i+1}, j=2, \ldots J(i+1)$.

The construction implies that for each fixed $j$, the sequence $\left\{T_{j}^{i}\right\}_{i=1}^{\infty}$ of currents is either increasing (in the obvious sense) or else equals zero after a finite number of steps. In addition, for every fixed $J$ the sequence $\left\{\sum_{j=1}^{J} T_{j}^{i}\right\}_{i=1}^{\infty}$ is increasing. It follows that $R_{j}=\lim _{i \rightarrow \infty} T_{j}^{i}$ exists for every $j$, and each $R_{j}$ is the current associated with integration over a (possibly infinite) Lipschitz curve. One can also pass to limits to find that

$$
\left\|T^{\prime}\right\|=\sum\left\|R_{j}\right\|, \quad\left\|\partial T^{\prime}\right\|=\sum_{j}\left\|\partial R_{j}\right\|
$$

as measures, since this holds in every compact subset. Thus the decomposition $T=\sum T_{X_{i}}+\sum R_{j}$ satisfies the conclusions of the lemma.

3. The conclusions about currents $T \in \mathcal{R}_{1, \rho}$ follow immediately from the earlier conclusions. 
I now present the

Proof of Lemma 6. First,

$$
L\left(p_{\#} T\right)=p_{\#} T\left(\rho^{2} \mathrm{~d} x^{3}\right)=T\left(p^{\#}\left(\rho^{2} \mathrm{~d} x^{3}\right)\right)
$$

But since $p^{3}\left(x_{1}, x_{2}, x_{3}\right)=x_{3}$ and $\rho(p(x))=\rho(x)$ for all $x, p^{\#}\left(\rho^{2} \mathrm{~d} x^{3}\right)=\rho^{2} \mathrm{~d} x^{3}$, proving the first claim.

If $X:(a, b) \rightarrow \mathcal{D}$ is a Lipschitz curve,

$$
E_{0}\left(p_{\#} T_{X}\right)=E_{0}\left(T_{p(X)}\right)=\int_{a}^{b} \rho\left(p(X)\left|\frac{\mathrm{d}}{\mathrm{d} t} p \circ X\right|=\int_{a}^{b} \rho(X)|\nabla p(X) \dot{X}| .\right.
$$

One can check by direct calculation that for $x \in \mathcal{D}$ and $v \in \mathbb{R}^{3}$,

$$
|\nabla p(x) v| \leq|v|, \quad \text { with equality iff } \begin{cases}x_{1}=0 \text { or } v=\left(0,0, v_{3}\right) & \text { if } a_{1}<a_{2} \\ \left(-x_{2}, x_{1}, 0\right) \cdot v=0 & \text { if } a_{1}=a_{2} .\end{cases}
$$

It follows that $E_{0}\left(p_{\#} T\right) \leq E_{0}(T)$. The conditions for equality (4.3) in the case $a_{1}<a_{2}$ follow easily from (9.5). In the case $a_{1}=a_{2},(9.5)$ shows that equality holds if and only if $\left(-X_{2}, X_{1},\right) \cdot\left(\dot{X}_{1}, \dot{X}_{2}, \dot{X}_{3}\right)=0$ almost everywhere, which implies that $\frac{\mathrm{d}}{\mathrm{d} t}\left(X_{1} / X_{2}\right)=0$. Thus there exist constants $\nu_{1}, \nu_{2}$ with $\nu_{1}^{2}+\nu_{2}^{2}=1$, such that $X_{1}(t) \nu_{1}+X_{2}(t) \nu_{2}=0$ for all $t$, and this is equivalent to (4.4).

For general $T \in \stackrel{\circ}{\mathcal{R}}_{1, \rho}(\mathcal{D})$, we use Lemma 3 to write $T$ in the form $T=\sum_{i} T_{X_{i}}$, where each $T_{X_{i}} \in \stackrel{\circ}{\mathcal{R}}_{1, \rho}$ corresponds to integration along a Lipschitz curve $X_{i}$, and such that $E_{\Omega}(T)=\sum_{i} E_{\Omega}\left(T_{X_{i}}\right)$. From this and the case of a single Lipschitz curve it follows that

$$
E_{0}(T)=\sum_{i} E_{0}\left(T_{X_{i}}\right) \geq \sum_{i} E_{0}\left(p_{\#} T_{X_{i}}\right) \geq E_{0}\left(\sum_{i} p_{\#} T_{X_{i}}\right)=E_{0}\left(p_{\#} T\right) .
$$

I now give the

Proof of Lemma 7. In [1], Theorem 4, this is proved for indecomposable currents $T$ of the form $T_{X}$ in $\dot{\mathcal{R}}_{1, \rho}\left(\mathcal{D}_{0}\right)$.

If $T$ is an arbitrary current in $\stackrel{\circ}{\mathcal{R}}_{1, \rho}\left(\mathcal{D}_{0}\right)$, then the result follows by using the decomposition $T=\sum T_{X_{i}}$ and arguing as in the proof of the previous lemma.

Finally, for a current $T \in \stackrel{\circ}{\mathcal{R}}_{1, \rho}(\mathcal{D})$,

$$
|L(T)|=\left|L\left(p_{\#} T\right)\right| \leq C E_{0}\left(p_{\#} T\right)^{3 / 2} \leq C E_{0}(T)^{3 / 2}
$$

using the previous lemma and the fact that $p_{\#} T \in \stackrel{\circ}{\mathcal{R}}_{1, \rho}\left(\mathcal{D}_{0}\right)$.

Next I present the proof of a lemma used in establishing the $\Gamma$-limit upper bound:

\section{Lemma 15.}

$$
\mathcal{F}:=\left\{T: \frac{1}{\pi} T \in \dot{\mathcal{R}}_{1, \rho}, T \text { has finite mass }\right\}
$$

is dense in $\pi \mathcal{R}_{1, \rho}:=\left\{T: \frac{1}{\pi} T \in \stackrel{\circ}{\mathcal{R}}_{1, \rho}\right\}$ in the sense that for for every $T \in \pi \stackrel{\circ}{\mathcal{R}}_{1, \rho}$, there exists a sequence of currents $\left\{T_{\delta}\right\}_{\delta \in(0,1]} \subset \mathcal{F}$ such that

$$
\mathbf{F}_{p, \rho, K}\left(T_{\delta}-T\right) \rightarrow 0, \text { for all compact } K \subset \mathcal{D}
$$

and

$$
E_{\Omega}\left(T_{\delta}\right) \rightarrow E_{\Omega}(T)
$$


Proof. As in the proof of Theorem 2, for $\sigma>0$ let $\mathcal{D}_{\sigma}:=\left\{x \in \mathcal{D}: \rho(x)>\|\nabla \rho\|_{\infty} \sigma\right\}$, and for $\delta>0$, let $\zeta_{\delta}: \mathcal{D}_{2 \delta} \rightarrow \mathcal{D}$ be a diffeomorphism satisfying (6.14), (6.15), and (6.16). Given $J \in \pi \mathcal{R}_{1, \rho}$, define $J_{\delta}:=\left(\zeta_{\delta}\right)_{\#} J$, so that $J_{\delta}(\phi)=J\left(\zeta_{\delta}^{\#} \phi\right)$. In view of $(6.14)$,

$$
J_{\delta}(\phi)=\int_{\mathcal{D}_{2 \delta}}\left\langle\zeta_{\delta}^{\#} \phi, \vec{J}\right\rangle \mathrm{d}\|J\|=\int_{\mathcal{D}_{4 \delta}}\langle\phi, \vec{J}\rangle \mathrm{d}\|J\|+\int_{\mathcal{D}_{2 \delta} \backslash \mathcal{D}_{4 \delta}}\left\langle\zeta_{\delta}^{\#} \phi, \vec{J}\right\rangle \mathrm{d}\|J\| .
$$

I first verify that $J_{\delta}$ has finite mass for every $\delta>0$. Indeed, for every $\phi$ such that $\|\phi\|_{\infty} \leq 1$, the definition of $\zeta_{\delta}$ implies that $\left\|\zeta_{\delta}^{\#} \phi\right\|_{\infty} \leq C$, and so using (9.9),

$$
J_{\delta}(\phi) \leq \frac{\left\|\zeta_{\delta}^{\#} \phi\right\|_{\infty}}{2 \delta} \int_{\mathcal{D}_{2 \delta}} 2 \delta \mathrm{d}\|J\| \leq \frac{C}{2 \delta} \mathbf{M}_{\rho}(J)<\infty
$$

Because $\mathbf{M}\left(J_{\delta}\right)=\sup \left\{J_{\delta}(\phi): \phi \in C_{c}^{\infty}\left(\mathcal{D} ; \Lambda^{1} \mathbb{R}^{3}\right),|\phi(x)| \leq 1 \forall x\right\}$, this shows that $J_{\delta}$ has finite mass.

Next I claim that $\mathbf{M}_{\rho}\left(J_{\delta}\right) \rightarrow \mathbf{M}_{\rho}(J)$ as $\delta \rightarrow 0$. Note that

$$
\mathbf{M}_{\rho}\left(J_{\delta}\right)=\sup \left\{J_{\delta}(\phi): \phi \in C_{c}^{\infty}\left(\mathcal{D} ; \Lambda^{1} \mathbb{R}^{3}\right),\left\|\frac{\phi}{\rho}\right\|_{\infty} \leq 1\right\}
$$

Also, for any $\phi$, arguments from the proof of Lemma 14, using (6.16) and (6.15), show that $\left\|\zeta_{\delta}^{\#} \phi / \rho\right\|_{\infty} \leq$ $C\|\phi / \rho\|_{\infty}$, with a constant independent of $\delta$. Thus (9.9) and (9.10) imply that

$$
\mathbf{M}_{\rho}\left(J_{\delta}\right) \leq \int_{\mathcal{D}_{4 \delta}} \rho \mathrm{d}\|J\|+C \int_{\mathcal{D}_{2 \delta} \backslash \mathcal{D}_{4 \delta}} \rho \mathrm{d}\|J\| \rightarrow \mathbf{M}_{\rho}(J)
$$

as $\delta \rightarrow 0$, by the dominated convergence Theorem.

In particular, $\mathbf{M}_{\rho}\left(J_{\delta}\right) \leq C$, and so it follows from Lemma 2 that $\left\{J_{\delta}\right\}_{\delta \in(0,1]}$ is precompact in the $\mathbf{F}_{p, \rho, K}$ seminorm for all compact $K \subset \mathcal{D}$. It is easy to see that $J_{\delta} \rightarrow J$ in the sense of distributions, and so it follows that (9.7) holds.

To verify (9.8), note that we have already checked that $E_{0}\left(J_{\delta}\right) \rightarrow E_{0}(J)$, and so it suffices to show that $J_{\delta}\left(\rho^{2} \mathrm{~d} x^{3}\right) \rightarrow J\left(\rho^{2} \mathrm{~d} x^{3}\right)$. However this can be easily deduced from (9.9).

Finally,

Lemma 16. If $T \in \dot{\mathcal{M}}_{1, \rho}(\mathcal{D})$ then for every compact $K \subset \mathcal{D}$ there exists a constant $C_{K}$ such that

$$
\mathbf{F}_{p, \rho, K}(T) \leq C_{K} \mathbf{F}_{\rho}(T)=C_{K} \inf \left\{\mathbf{M}_{\rho}(S): \partial S=T\right\} .
$$

Proof. 1. First I claim that $\mathbf{M}_{\rho}\left(p_{\#} S\right) \leq \mathbf{M}_{\rho}(S)$ for all $S \in \mathcal{M}_{2, \rho}(\mathcal{D})^{7}$. Recall that $p: \mathcal{D} \rightarrow \mathcal{D}_{0}$ is Lipschitz with Lipschitz constant 1 , and $\rho(p(x))=\rho(x)$ for all $x \in \mathcal{D}$. Thus for any $k$-form $\phi$ on $\mathcal{D}_{0}$,

$$
\frac{\left|p^{\#} \phi(x)\right|}{\rho(x)} \leq \frac{|\phi(p(x))|}{\rho(p(x))}
$$

and so $\left\|p^{\#} \phi / \rho\right\|_{\infty} \leq\|\phi / \rho\|_{\infty}$. The claim follows from this and the expression for the weighted mass $\mathbf{M}_{\rho}$ given in (3.6). Note also that for $T \in \dot{\mathcal{M}}_{1, \rho}(\mathcal{D})$, if $T=\partial S$ then $p_{\#} T=\partial p_{\#} S$. Thus

$$
\mathbf{F}_{p, \rho}(T) \leq \inf \left\{\mathbf{M}_{\rho}\left(p_{\#} S\right): \partial S=T\right\} \leq \inf \left\{\mathbf{M}_{\rho}(S): \partial S=T\right\} \leq \mathbf{F}_{\rho}(T) .
$$

\footnotetext{
${ }^{7}$ The same fact was proved (along with conditions for equality) in Lemma 6 for $\mathcal{R}_{1, \rho}$; the proof we give here is easy and general, but it is harder to extract from it the conditions for equality.
} 
2. For compact $K \subset \mathcal{D}$, there exists some $C$ such that $\rho \geq C^{-1}$ on $K$, which implies that $\mathbf{M}_{K}(S) \leq C \mathbf{M}_{\rho}(S)$ for all currents $T$. This immediately implies that

$$
\mathbf{F}_{K}(T)=\inf \left\{\mathbf{M}_{K}(S): \partial S=T \text { in } K\right\} \leq C \inf \left\{\mathbf{M}_{\rho}(S): \partial S=T\right\} \leq C \mathbf{F}_{\rho}(T)
$$

Acknowledgements. This research was partially supported by the National Science and Engineering Research Council of Canada under Operating Grant 261955. I am grateful to Amandine Aftalion for helpful discussions.

\section{REFERENCES}

[1] A. Aftalion and R.L. Jerrard, On the shape of vortices for a rotating Bose-Einstein condensate. Phys. Rev. A 66 (2002) 023611.

[2] A. Aftalion and R. L. Jerrard, Properties of a single vortex solution in a rotating Bose-Einstein condensate. C. R. Acad. Sci. Paris Ser. I 336 (2003) 713-718.

[3] A. Aftalion and T. Rivière, Vortex energy and vortex bending for a rotating Bose-Einstein condensate. Phys. Rev. A 64 (2001) 043611.

[4] G. Alberti, S. Baldo and G. Orlandi, Functions with prescribed singularities. J. Eur. Math. Soc. 5 (2003) 275-311.

[5] G. Alberti, S. Baldo and G. Orlandi, Variational convergence for functionals of Ginzburg-Landau type. Indiana Univ. Math J. 54 (2005) 1411-1472.

[6] N. Andre and I. Shafrir, Asymptotic behavior of minimizers for the Ginzburg-Landau functional with weight. I, II. Arch. Rational Mech. Anal. 142 (1998) 45-73, 75-98.

[7] F. Bethuel, H. Brezis and F. Hélein, Ginzburg-Landau Vortices. Birkhauser, New-York (1994).

[8] H. Brezis, J.M. Coron, and E.H. Lieb, Harmonic maps with defects. Comm. Math. Phys. 107 (1986) 649-705.

[9] L.C. Evans and R.F. Gariepy, Measure Theory and Fine Properties of Functions. CRC Press, London (1992).

[10] H. Federer, Geometric Measure Theory. Springer-Verlag, Berlin (1969).

[11] M. Giaquinta, G. Modica and J. Soucek, Cartesian Currents in the Calculus of Variations. I, II. Springer-Verlag, New York (1998).

[12] R.L. Jerrard and H.M. Soner, The Jacobian and the Ginzburg-Landau functional. Cal. Var. 14 (2002) 151-191.

[13] R.L. Jerrard, A. Montero, and P. Sternberg, Local minimizers of the Ginzburg-Landau energy with magnetic field in three dimensions. Comm. Math. Phys. 249 (2004) 549-577.

[14] R.V. Kohn and P. Sternberg, Local minimizers and singular perturbations. Proc. Royal Soc. Edin. 111A (1989) 69-84.

[15] L. Lassoued and P. Mironescu, Ginzburg-Landau type energy with discontinuous constraint. J. Anal. Math. 77 (1999) 1-26.

[16] A. Montero, P. Sternberg, and W. Ziemer, Local minimizers with vortices to the Ginzburg-Landau system in 3-d. Comm. Pure Appl. Math 57 (2004) 99-125.

[17] C. Raman, J. R. Abo-Shaeer, J. M. Vogels, K. Xu and W. Ketterle, Vortex nucleation in a stirred Bose-Einstein condensate. Phys. Rev. Lett. 87 (2001) 210402.

[18] T. Rivière, Line vortices in the U(1)-Higgs model. Cont. Opt. Calc. Var. 1 (1996) 77-167.

[19] P. Rosenbuch, V. Bretin, and J. Dalibard, Dynamics of a single vortex line in a Bose-Einstein condensate. Phys. Rev. Lett. 89 (2002) 200403.

[20] E. Sandier and S. Serfaty. A product estimate for Ginzburg-Landau and corollaries. J. Funct. Anal. 211 (2004) $219-244$. 